\title{
1 Singlet-triplet excitation energies of substituted benzenes: \\ 2 A G4 theoretical study
}

3

4 Sierra Rayne $^{\mathrm{a}, *}$ and Kaya Forest ${ }^{\mathrm{b}}$

$6 \quad{ }^{a}$ Chemologica Research, PO Box 74, 318 Rose Street, Mortlach, Saskatchewan, Canada, S0H 3E0

$7 \quad{ }^{b}$ Department of Environmental Engineering, Saskatchewan Institute of Applied Science and

8 Technology, Palliser Campus, PO Box 1420, 600 6th Avenue NW, Moose Jaw, Saskatchewan, Canada,

$9 \mathrm{~S} 6 \mathrm{H} 4 \mathrm{R} 4$

10

11 "Corresponding author. Tel.: +1 306690 0573. E-mail address: rayne.sierra@gmail.com (S. Rayne). 


\section{Abstract}

15 Singlet-triplet adiabatic $\left(\mathrm{AE}_{\mathrm{S}-\mathrm{T}}\right)$ and well-to-well $\left(\mathrm{WWE}_{\mathrm{S}-\mathrm{T}}\right)$ excitation energies of variously substituted 16 phenyl cations were calculated at the G4 level of theory. The G4 $\mathrm{E}_{\mathrm{S}-\mathrm{T}}$ estimates range from 19 to 40

$17 \mathrm{~kJ} / \mathrm{mol}$ higher than prior density functional theory based predictions for these cations, and suggest that $18 \mathrm{E}_{\mathrm{S}-\mathrm{T}}$ and ground state multiplicity structure-property trends for phenyl cations previously proposed in

19 the literature may be incorrect. Among the substituents considered, meta-substituted phenyl cations

20 with electron donating groups have singlet ground states, and are not isoenergetic with the

21 corresponding triplet states as previously claimed. Depending on the functional group, ortho- or para-

22 substituted phenyl cations may also be ground state singlets despite the presence of an electron

23 donating moiety.

25 Keywords: Phenyl cations; Substituent effects; Singlet-triplet excitation energies; Ground state 26 multiplicity 
The existence and energetics of aryl cations has long been a topic of substantial interest among the organic chemistry community [1-16]. In particular, the nature of the ground state multiplicity, and magnitude of the singlet-triplet excitation energy $\left(\mathrm{E}_{\mathrm{S}-\mathrm{T}}\right)$, for substituted phenyl cations has attracted a modicum of theoretical attention [17-19]. For example, in a recent study, Lazzaroni et al. [17] calculated the $\mathrm{E}_{\mathrm{S}-\mathrm{T}}$ for the parent and variously substituted (o-, m-, and p- $\mathrm{CH}_{3}, \mathrm{OCH}_{3}, \mathrm{NH}_{2}, \mathrm{CN}, \mathrm{NO}_{2}$, $\mathrm{SCH}_{3}$, and $\left.\mathrm{Si}\left(\mathrm{CH}_{3}\right)_{3}\right)$ phenyl cations at the UB3LYP/6-31G(d) and UB3LYP/6-311++G(2d,p) levels of theory (and subsequently utilized these findings in ref. [20]). The authors reported that "with electrondonating substituents, the triplet is the ground state for ortho and para derivatives, while the two spin states are roughly isoenergetic when the donating group is in the meta position." Other groups have also calculated the Es-T $_{\text {s }}$ of substituted phenyl cations using density functional theory (DFT) methods $[18,19]$, and made inferences regarding ground state multiplicities based on the computational results. However, DFT methods, particularly using the B3LYP functional, are known to significantly underestimate the $\mathrm{E}_{\text {s-T }}$ of organic compounds, and the use of higher-level (e.g., composite) theoretical methods and/or more modern density functionals is required in order to achieve both qualitative and quantitative $\mathrm{E}_{\mathrm{S}-\mathrm{T}}$ predictivity (see, ref. [9, 21-27], and references therein).

In light of these issues, we chose to re-examine the $\mathrm{E}_{\mathrm{S}-\mathrm{T}}$ of several representative substituted phenyl cations using the G4 [28] and G4MP2 [29] composite methods as employed in Gaussian 09 [30]. All calculations were conducted in the gas phase (1 atm) at $298.15 \mathrm{~K}$. Geometries were visualized using Gabedit 2.3.9 [31] and Avogadro 1.01 (http://avogadro.openmolecules.net/). Except where noted otherwise, all compounds converged absent imaginary frequencies. The substituents investigated at the $\mathrm{o}-, \mathrm{m}-$, and $\mathrm{p}$ - positions included the cyano, amino, nitro, thiomethoxy, and methoxy moieties considered by Lazzaroni et al. [17], as well as bromo, chloro, ethynyl, and dimethylamino groups. For the o- and m-methoxy and thiomethoxy substituted phenyl cations, both syn- and anti-conformations are possible in the singlet and triplet states (Figure 1). Based on the geometries presented in their Supporting Information, Lazzaroni et al. [17] appear to have only calculated the energetics of the synconformations for these cations. G4 converged geometries with one imaginary frequency are present for the triplet syn-o-methoxyphenyl cation and the singlet anti-o-methoxyphenyl cation, precluding ES-T $_{\mathrm{T}}$ calculations on these structures. Analogous results were obtained at the G4MP2 level.

At the G4MP2 level, we find the singlet anti-o-thiomethoxyphenyl cation is $0.9 \mathrm{~kJ} / \mathrm{mol}$ lower in energy than the syn-conformer, equivalent to the G4 energy difference of $1.0 \mathrm{~kJ} / \mathrm{mol}$. In contrast, the triplet syn-o-thiomethoxyphenyl cation is $5.4 / 5.6(\mathrm{G} 4 \mathrm{MP} 2 / \mathrm{G} 4) \mathrm{kJ} / \mathrm{mol}$ lower in energy than the anticonformer. Thus, both G4MP2 and G4 calculations suggest the dominant singlet-triplet transition for the o-thiomethoxyphenyl cation would be from an anti-o-thiomethoxyphenyl singlet state to a syn-othiomethoxyphenyl triplet state (or vice versa, depending on the ground state of the system). At the G4MP2 level, for the m-thiomethoxyphenyl (singlet syn $\rightarrow$ anti isomerization enthalpy $\left[\Delta_{\text {isom }} \mathrm{H}_{(\mathrm{g})}^{\circ}\right]=+5.1$ $\mathrm{kJ} / \mathrm{mol}$; triplet syn $\rightarrow$ anti $\Delta_{\text {isom }} \mathrm{H}_{(\mathrm{g})}^{\circ}=+1.5 \mathrm{~kJ} / \mathrm{mol}$ ) and m-methoxyphenyl (singlet syn $\rightarrow$ anti $\Delta_{\text {isom }} \mathrm{H}_{(\mathrm{g})}^{\circ}=+2.0 \mathrm{~kJ} / \mathrm{mol}$; triplet syn $\rightarrow$ anti $\Delta_{\text {isom }} \mathrm{H}^{\circ}{ }_{(\mathrm{g})}=+5.1 \mathrm{~kJ} / \mathrm{mol}$ ) phenyl cations, the syn-conformations are lower in energy than the corresponding anti-geometries. In addition to these substituents, we also conducted G4 E $_{\text {-T }}$ calculations on the p-hydroxy and thiolphenyl cations, but the singlet states of both compounds contained one imaginary frequency.

Adiabatic $\left(\mathrm{AE}_{\mathrm{S}-\mathrm{T}}\right)$ and well-to-well $\left(\mathrm{WWE}_{\mathrm{S}-\mathrm{T}}\right)$ singlet-triplet excitation energies at $298.15 \mathrm{~K}$ and $1 \mathrm{~atm}$ using the G4 composite method are presented in Table 1 for the parent and substituted phenyl cations under study, along with Es-T data from ref. [17-19]. The G4 values, which are likely more accurate than the previously published DFT results, are systematically higher than the DFT values by 19 to 40 
$\mathrm{kJ} / \mathrm{mol}$. Of greater import, the G4 $\mathrm{E}_{\mathrm{S}-\mathrm{T}}$ are in qualitative disagreement with the DFT data regarding the ground state multiplicity of some phenyl cations with electron donating substituents. Lazzaroni et al. [17] predicted that the o-, m-, and p-amino and thiomethoxyphenyl cations would all be triplet ground states, whereas the G4 calculations predict only the o- and p-isomers will be triplet ground states, whereas the $\mathrm{m}$-isomers will clearly $(+18 \mathrm{~kJ} / \mathrm{mol}$ for the $\mathrm{m}$-amino and $+17 \mathrm{~kJ} / \mathrm{mol}$ for the $\mathrm{m}$ thiomethoxy) be singlet ground states. Similarly, Lazzaroni et al. [17] predicted the p-methoxyphenyl cation to be a triplet ground state (by almost $20 \mathrm{~kJ} / \mathrm{mol}$ ), while the $\mathrm{G} 4$ results predict a singlet ground state (by about $4-6 \mathrm{~kJ} / \mathrm{mol}$ ).

Given the range in $E_{\text {s-T }}$ differences between our G4 results and the DFT data of Lazzaroni et al. [17], although neither G4MP2 nor G4 E-T calculations could be completed on the o-methoxyphenyl cation due to the presence of imaginary frequencies, there is ambiguity regarding the ground state of this cation as well (i.e., the UB3LYP/6-31G(d) E S-T $_{\text {T }}$ underestimate for the o-methoxyphenyl cation may be greater than $32 \mathrm{~kJ} / \mathrm{mol}$, in which case the ground state would be a singlet). Alternatively, the presence of the imaginary frequencies at the G4MP2 and G4 levels may not be artifacts of the computational methods, and these compounds may not exist in the gas phase at ambient temperature for a finite lifetime. Similarly, the presence of G4 level singlet state imaginary frequencies for the p-hydroxy and thiophenyl cations not only prevents comparison with the $\mathrm{E}_{\mathrm{S}-\mathrm{T}}$ estimates for these compounds in ref. $[18,19]$, but also questions whether they have a gas phase singlet state potential energy well.

As Bondarchuk and Minaev [16] have recently shown at the B3LYP/6-31G (d,p) level of theory, care needs to be taken when investigating phenyl cations via theoretical methods. In this study, the authors show that the singlet o-nitrophenyl cation cannot likely exist in the gas phase, as calculations lead to a rearranged structure. At the G4 level, we are able to reproduce the gas phase findings of Bondarchuk and Minaev [16] for the o-nitrophenyl cation (see Supplementary Material for structure), while the mand p-nitrophenyl cations give converged structures using the G4 method with no imaginary frequencies and absent any intramolecular rearrangements. However, the triplet states of the o-, m-, and p-nitrophenyl cations all yield one imaginary frequency at the G4 level, preventing comparison with the $\mathrm{E}_{\mathrm{S}-\mathrm{T}}$ estimates of Lazzaroni et al. [17] for these cations (the o-nitrophenyl cation $\mathrm{E}_{\mathrm{S}-\mathrm{T}}$ data was not provided in the Supporting Information of ref. [17], but values of 79.0 and $65.4 \mathrm{~kJ} / \mathrm{mol}$ for the $\mathrm{m}$ - and $\mathrm{p}$ - isomers is available from these authors).

In general, the G4 calculations presented herein suggest that the singlet-triplet excitation energy and ground state multiplicity structure-property trends for phenyl cations previously proposed in the literature may be incorrect. It appears that rather than meta-substituted phenyl cations with electron donating groups having approximately isoenergetic singlet and triplet states, it is likely the ground state is clearly a singlet (at least within the confines of the substituents examined herein). Furthermore, not all ortho-/para-substituted phenyl cations with electron donating groups are ground state triplets; in some cases, either the ortho- or para- isomer may be a ground state singlet despite the presence of an electron donating group. In addition, several substituted phenyl cation singlet and/or triplet states may not be minima on their respective potential energy surfaces when examined at higher levels of theory, thereby precluding the formal calculation of singlet-triplet energy gaps. 


\section{Acknowledgements}

119

120 This work was made possible by the facilities of the Western Canada Research Grid (WestGrid: project

121 100185), the Shared Hierarchical Academic Research Computing Network (SHARCNET: project

122 sn4612), and Compute/Calcul Canada.

123

124

125

126 Supplementary data is associated with this article.

127 


\section{Figure Captions}

129

130 Figure 1. Structures of the syn- and anti-conformations for the o-(thio)methoxyphenyl cations. 131 
132 Figures

133

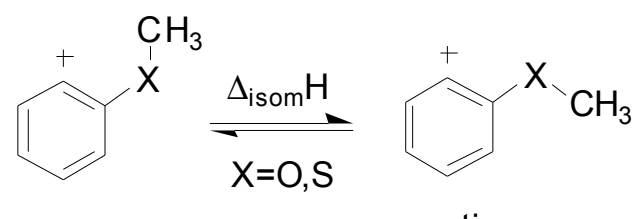

134 syn anti

135

136 Figure 1.

137 


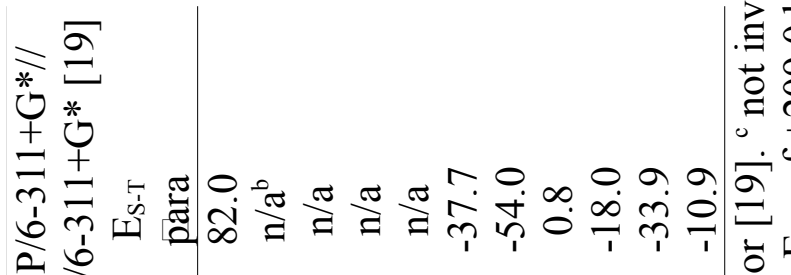

至

焉

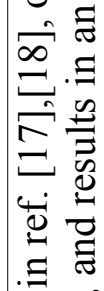

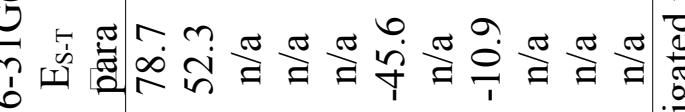

空

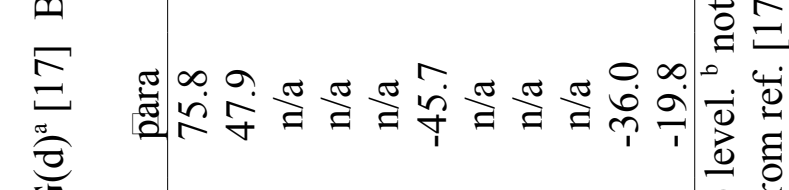

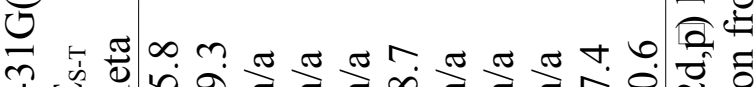

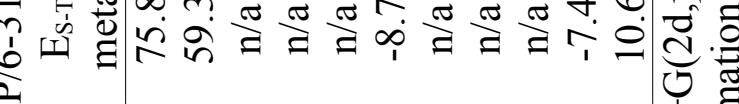

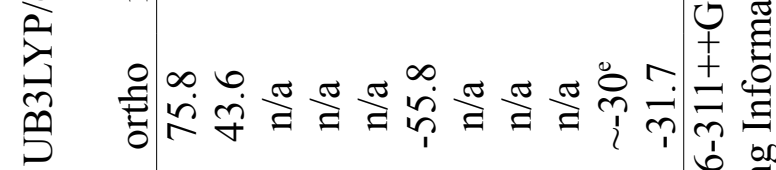

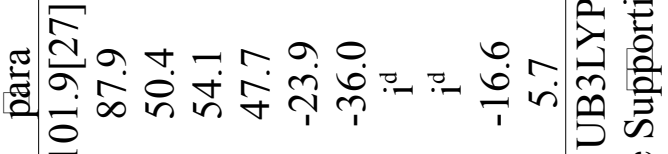

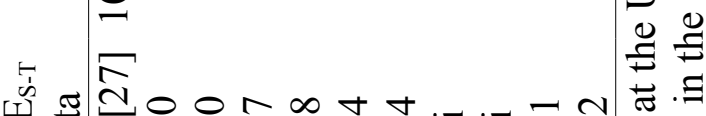

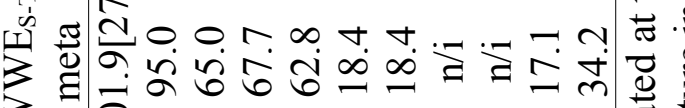
घ

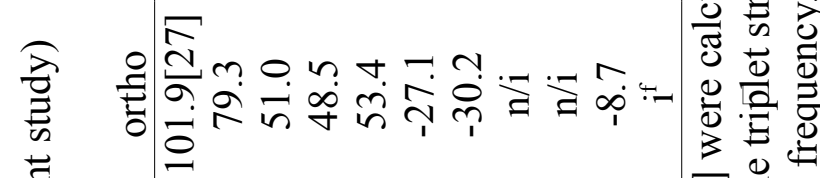

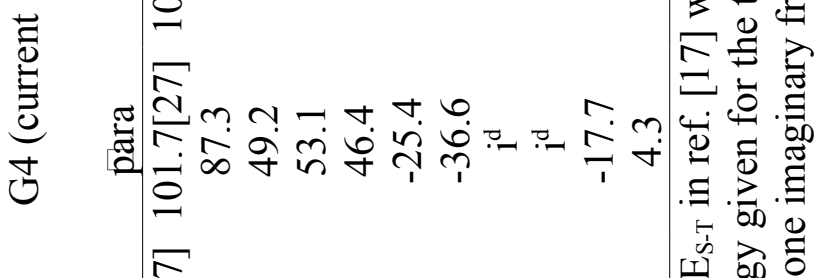

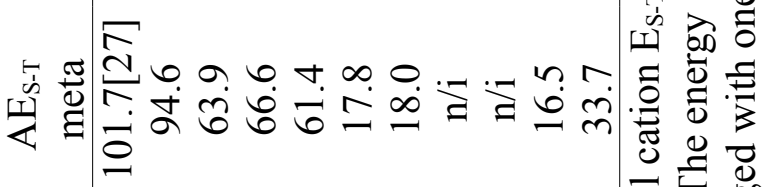

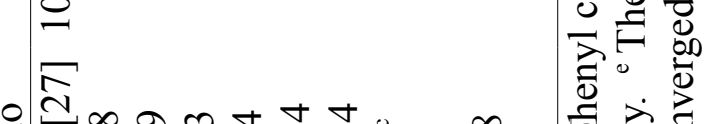

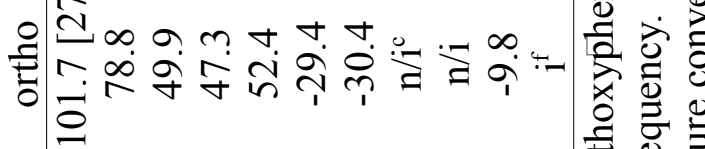

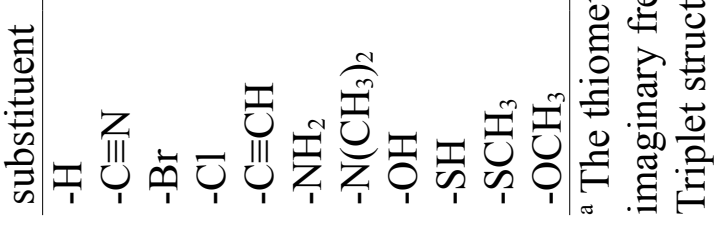




\section{References}

[1] W.A. Waters, 50. Decomposition reactions of the aromatic diazo-compounds. Part X. Mechanism of the Sandmeyer reaction, J. Chem. Soc. (1942) 266-270.

[2] R.W. Taft, Evidence for phenyl cation with an odd number of $\pi$-electrons from the aqueous thermal decomposition of the diazonium ion, J. Am. Chem. Soc. 83 (1961) 3350-3351.

[3] H. Zollinger, Reactivity and stability of arenediazonium ions, Acc. Chem. Res. 6 (1973) 335-341.

[4] J.D. Dill, P.V.R. Schleyer, J.S. Binkley, R. Seeger, J.A. Pople, E. Hasselbach, Molecular orbital theory of the electronic structure of molecules. 30. Structure and energy of the phenyl cation, J. Am. Chem. Soc. 98 (1976) 5428-5431.

[5] H.B. Ambroz, T.J. Kemp, Aryl cations - new light on old intermediates, Chem. Soc. Rev. 8 (1979) 353-365.

[6] C.G. Swain, J.E. Sheats, K.G. Harbison, Evidence for phenyl cation as an intermediate in reactions of benzenediazonium salts in solution, J. Am. Chem. Soc. 97 (1975) 783-790.

[7] G. Angelini, S. Fornarini, M. Speranza, Gas- and liquid-phase reaction of free phenylium cation with methanol, J. Am. Chem. Soc. 104 (1982) 4773-4780.

[8] S.J. Klippenstein, $\mathrm{Ab}$ initio reaction path energetics for the $\mathrm{CX}$ dissociations of $\mathrm{C}_{6} \mathrm{H}_{5} \mathrm{X}^{+}$with $\mathrm{X}=\mathrm{H}$, F, Cl, and Br, Int. J. Mass. Spectrom. 167/168 (1997) 235-257.

[9] A. Nicolaides, D.M. Smith, F. Jensen, L. Radom, Phenyl radical, cation, and anion. The tripletsinglet gap and higher excited states of the phenyl cation, J. Am. Chem. Soc. 119 (1997) 8083-8088. [10] S.M. Gasper, C. Devadoss, G.B. Schuster, Photolysis of substituted benzenediazonium salts: Spinselective reactivity of aryl cations, J. Am. Chem. Soc. 117 (1995) 5206-5211.

[11] J. Hrusak, D. Schroder, S. Iwata, The ground state $\left({ }^{1} \mathrm{~A}_{1}\right)$ and the lowest triplet state $\left({ }^{3} \mathrm{~B}_{1}\right)$ of the phenyl cation $\mathrm{C}_{6} \mathrm{H}_{5}^{+}$revisited, J. Chem. Phys. 106 (1997) 7541-7549.

[12] M. Winkler, W. Sander, Generation and reactivity of the phenyl cation in cryogenic argon matrices: Monitoring the reactions with nitrogen and carbon monoxide directly by IR spectroscopy, J. Org. Chem. 71 (2006) 6357-6367.

[13] M. Slegt, F. Minne, H. Zuilhof, H.S. Overkleeft, G. Lodder, Photochemical generation and reactivity of naphthyl cations: Cine substitution, Eur. J. Org. Chem. 32 (2007) 5353-5363.

[14] H.A. Galue, J. Oomens, Spectroscopic evidence for a triplet ground state in the naphthyl cation, Angew Chem. Int. Edit. 50 (2011) 7004-7007.

[15] A. Patzer, S. Chakraborty, N. Solca, O. Dopfer, IR spectrum and structure of the phenyl cation, Angew. Chem. Int. Ed. 49 (2010) 10145-10148.

[16] S.V. Bondarchuk, B.F. Minaev, Density functional study of ortho-substituted phenyl cations in polar medium and in the gas phase, Chem. Phys. (2011) doi:10.1016/j.chemphys.2011.08.005.

[17] S. Lazzaroni, D. Dondi, M. Fagnoni, A. Albini, Geometry and energy of substituted phenyl cations, J. Org. Chem. 73 (2008) 206-211.

[18] M. Aschi, J.N. Harvey, Spin isomerisation of para-substituted phenyl cations, J. Chem. Soc., Perkin Trans. 2 (1999) 1059-1062.

[19] K.K. Laali, G. Rasul, G.K.S. Prakash, G.A. Olah, DFT study of substituted and benzannelated aryl cations: Substituent dependency of singlet/triplet ratio, J. Org. Chem. 67 (2002) 2913-2918.

[20] S. Lazzaroni, D. Dondi, M. Fagnoni, A. Albini, Selectivity in the reaction of triplet phenyl cations, J. Org. Chem. 75 (2010) 315-323.

[21] S. Gronert, J.R. Keeffe, R.A.M. O'Ferrall, Stabilities of carbenes: Independent measures for singlets and triplets, J. Am. Chem. Soc. 133 (2011) 3381-3389.

[22] H.L. Woodcock, D. Moran, B.R. Brooks, P.V.R. Schleyer, H.F. Schaefer, Carbene stabilization by aryl substituents. Is bigger better?, J. Am. Chem. Soc. 129 (2007) 3763-3770. 
192 [23] X. Zhou, D.A. Hrovat, R. Gleiter, W.T. Borden, Reinvestigation of the ordering of the low-lying 193 electronic states of cyclobutanetetraone with CASPT2, CCSD(T), G3B3, ccCA, and CBS-QB3

194 calculations, Mol. Phys. 107 (2009) 863-870.

195 [24] A.H. Winter, D.E. Falvey, Vinyl cations substituted with $\beta \pi$-donors have triplet ground states, J.

196 Am. Chem. Soc. 132 (2010) 215-222.

197 [25] S. Rayne, K. Forest, A comparison of density functional theory (DFT) methods for estimating the

198 singlet-triplet $\left(\mathrm{S}_{0}-\mathrm{T}_{1}\right)$ excitation energies of benzene and polyacenes, Comput. Theor. Chem. (2011) doi:

199 10.1016/j.comptc.2011.08.010.

200 [26] S. Rayne, K. Forest, Singlet-triplet $\left(\mathrm{S}_{0} \rightarrow \mathrm{T}_{1}\right)$ excitation energies of the $[4 \times \mathrm{n}]$ rectangular graphene 201 nanoribbon series ( $\mathrm{n}=2-6)$ : A comparative theoretical study, Comput. Theor. Chem. (2011) in press.

202 [27] S. Rayne, K. Forest, Singlet-triplet excitation energies of naphthyl cations: High level composite 203 method calculations suggest a singlet ground state, Comput. Theor. Chem. (2011) submitted.

204 [28] L.A. Curtiss, P.C. Redfern, K. Raghavachari, Gaussian-4 theory, J. Chem. Phys. 126 (2007) 205 84108-84112.

206 [29] L.A. Curtiss, P.C. Redfern, K. Raghavachari, Gaussian-4 theory using reduced order perturbation 207 theory, J. Chem. Phys. 127 (2007) 124105-124108.

208 [30] M.J. Frisch, G.W. Trucks, H.B. Schlegel, G.E. Scuseria, M.A. Robb, J.R. Cheeseman, G.

209 Scalmani, V. Barone, B. Mennucci, G.A. Petersson, et al., Gaussian 09, Revision B.01, Gaussian, Inc., 210 Wallingford, CT, 2010.

211 [31] A.R. Allouche, Gabedit: A graphical user interface for computational chemistry softwares, J.

212 Comput. Chem. 32 (2011) 174-182.

213 
Supplementary Material

Singlet-triplet excitation energies of substituted benzenes:

A G4 theoretical study

Sierra Rayne ${ }^{a, *}$ and Kaya Forest ${ }^{b}$

a Chemologica Research, PO Box 74, 318 Rose Street, Mortlach, Saskatchewan, Canada, $\mathrm{SOH} \quad 3 \mathrm{EO}$

b Department of Environmental Engineering, Saskatchewan Institute of Applied

Science and Technology, Palliser Campus, PO Box 1420, 600 6th Avenue NW, Moose Jaw, Saskatchewan, Canada, S6H 4R4

* Corresponding author. Tel.: +1 306690 0573. E-mail address: rayne.sierra@gmail.com (S. Rayne). 


\section{G4 archive entries}

${ }^{1}$ m-aminophenyl cation

$\backslash \backslash 1,1 \backslash \mathrm{C}, 0,-3.0628456473,2.990937019,-0.2910219173 \backslash \mathrm{C}, 0,-4.0203558187,2$. $0432631885,-0.8708644564 \backslash \mathrm{C}, 0,-1.7952297487,2.4870584871,0.146933257 \backslash \mathrm{C}$, $0,-1.4881385878,1.1513157382,0.107725841 \backslash \mathrm{C}, 0,-3.5255414109,0.829408682$ $5,-0.7230211998 \backslash \mathrm{C}, 0,-2.4985628546,0.1617027686,-0.2386026425 \backslash \mathrm{H}, 0,-1.07$ $15616574,3.1900372529,0.543944751 \backslash \mathrm{H}, 0,-2.4686118549,-0.8445401251,0.15$ $89965886 \backslash \mathrm{H}, 0,-0.5659132945,0.7816344632,0.5457870241 \backslash \mathrm{H}, 0,-4.7426714392$ $, 2.3743771796,-1.6062227865 \backslash \mathrm{N}, 0,-3.2885433196,4.2982614979,-0.41392411$ $95 \backslash \mathrm{H}, 0,-4.2086080471,4.6601615627,-0.6171602872 \backslash \mathrm{H}, 0,-2.5333363194,4.96$ $77322849,-0.3772800526 \backslash \backslash$ Version=EM64L-G09RevB.01 $\backslash$ State=1-A $\backslash \mathrm{MP} 2 / \mathrm{GTB}$ as $1=$ $-285.7078356 \backslash \mathrm{MP} 4 / \mathrm{GTBas} 1=-285.7952975 \backslash \mathrm{CCSD}(\mathrm{T}) / \mathrm{G} 3 \mathrm{Bas} 1=-285.7942251 \backslash \mathrm{MP} 2 / \mathrm{G}$ TBas $2=-285.7189758 \backslash \mathrm{MP} 4 / \mathrm{GTBas} 2=-285.8067298 \backslash \mathrm{MP} 2 / \mathrm{GTB}$ s $3=-285.911581 \backslash \mathrm{MP} 4 /$ GTBas $3=-286.0082052 \backslash \mathrm{HF} / \mathrm{GTLargeXP}=-284.8909403 \backslash \mathrm{MP} 2 / \mathrm{GTLargeXP}=-286.32508$ $58 \backslash \mathrm{HF} / \mathrm{GFHFB} 1=-284.9138173 \backslash \mathrm{HF} / \mathrm{GFHFB} 2=-284.9180031 \backslash \mathrm{G} 4=-286.4651771 \backslash \mathrm{FreqC}$ oord $=-5.7879394607,5.6520518467,-0.5499517224,-7.597371454,3.861207843$ $7,-1.6456953214,-3.3924925707,4.699859417,0.2776636155,-2.8121743786,2$ $.1756714377,0.2035723369,-6.6623077368,1.5673552621,-1.3663120559,-4.7$ $215995209,0.3055739476,-0.4508936488,-2.024958067,6.0282967617,1.02790$ $66108,-4.6650003342,-1.5959495447,0.3004600086,-1.0694211415,1.4770750$ $715,1.0313880024,-8.9623501583,4.4869226055,-3.0353211749,-6.214446250$ $2,8.1225370785,-0.7822032256,-7.9531166098,8.8064290886,-1.1662639229$, $-4.7873118462,9.38765352,-0.7129559747 \backslash \mathrm{PG}=\mathrm{C} 01 \quad[\mathrm{X}(\mathrm{C} 6 \mathrm{H} 6 \mathrm{~N} 1)] \backslash \mathrm{NImag}=0 \backslash \backslash$

${ }^{1}$ o-aminophenyl cation

$\backslash \backslash 1,1 \backslash \mathrm{C}, 0,-2.7378477779,2.7328466549,-0.3189998867 \backslash \mathrm{C}, 0,-3.7684684315,1$ $.8453438791,-0.3047278114 \backslash \mathrm{C}, 0,-1.5428009485,1.9137585941,-0.306067285 \backslash$ $\mathrm{C}, 0,-1.6108448204,0.5441531001,-0.1598293478 \backslash \mathrm{C}, 0,-4.0693298535,0.58707$ $12688,-0.0139580173 \backslash \mathrm{C}, 0,-2.8316290106,-0.1299254221,-0.0295825855 \backslash \mathrm{H}, 0$, $-0.602966792,2.454556346,-0.2178529329 \backslash \mathrm{H}, 0,-5.0274285546,0.0868291113$, $-0.0830628287 \backslash \mathrm{H}, 0,-2.9049668794,-1.2093562188,-0.1227589834 \backslash \mathrm{H}, 0,-0.690$ $0258712,-0.0266211543,-0.1160502625 \backslash N, 0,-2.7389774301,4.0719394735,-0$. $2614811326 \backslash \mathrm{H}, 0,-3.5709679557,4.5850420287,-0.0144197459 \backslash \mathrm{H}, 0,-1.8627156$ $747,4.5672923386,-0.2050691803 \backslash \backslash$ Version=EM64L-G0 9RevB.01 \State=1-A \MP2 $/ \mathrm{GTBas} 1=-285.6879541 \backslash \mathrm{MP} 4 / \mathrm{GTBas} 1=-285.7746306 \backslash \mathrm{CCSD}(\mathrm{T}) / \mathrm{G} 3 \mathrm{Bas} 1=-285.77445$ $09 \backslash \mathrm{MP} 2 / \mathrm{GTBas} 2=-285.6995669 \backslash \mathrm{MP} 4 / \mathrm{GTBas} 2=-285.7866269 \backslash \mathrm{MP} 2 / \mathrm{GTB}$ s $3=-285.893$ $4572 \backslash \mathrm{MP} 4 / \mathrm{GTBas} 3=-285.9894963 \backslash \mathrm{HF} / \mathrm{GTLargeXP}=-284.8794505 \backslash \mathrm{MP} 2 / \mathrm{GTLargeXP}=-$ $286.3069847 \backslash \mathrm{HF} / \mathrm{GFHFB1}=-284.9025022 \backslash \mathrm{HF} / \mathrm{GFHFB} 2=-284.9067197 \backslash \mathrm{G} 4=-286.4491$ 253 $\backslash$ FreqCoord=-5.1737824937, 5.1643317409,-0.6028224222, $-7.121373276,3$. $4871945525,-0.5758521087,-2.9154712701,3.6164796274,-0.5783833468,-3.0$ $440555531,1.0283003335,-0.3020336954,-7.6899189674,1.1094039185,-0.026$ $37683,-5.35100334,-0.2455234654,-0.055902985,-1.1394421041,4.638439271$ $6,-0.4116823805,-9.5004631208,0.1640832407,-0.156965998,-5.4895918271$, $-2.2853520505,-0.2319808591,-1.3039599212,-0.0503066909,-0.2193032138$, $-5.1759172271,7.6948504347,-0.4941277294,-6.7481514656,8.664473742,-0$. $0272493706,-3.5200224886,8.6309316888,-0.3875245891 \backslash \mathrm{PG}=\mathrm{C} 01 \quad[\mathrm{X}(\mathrm{C} 6 \mathrm{H} 6 \mathrm{~N} 1)]$ $\backslash$ NImag $=0 \backslash \backslash$

${ }^{1}$ p-aminophenyl cation

$\backslash \backslash 1,1 \backslash \mathrm{C}, 0,-2.893289216,3.0468266551,-0.2793221633 \backslash \mathrm{C}, 0,-4.105428994,2.3$ $091644731,-0.1309934458 \backslash \mathrm{C}, 0,-1.7032529513,2.4061344047,-0.6365413182 \backslash \mathrm{C}$ $, 0,-1.8959080587,1.0723217216,-0.5238323561 \backslash \mathrm{C}, 0,-4.1078137019,0.899168$ $2009,-0.3485576984 \backslash \mathrm{C}, 0,-2.9355623072,0.2270282232,-0.706791673 \backslash \mathrm{H}, 0,-2$. $8629564909,4.1095431528,-0.0599012994 \backslash \mathrm{H}, 0,-0.7669303484,2.9256020956,-$ $0.7973226282 \backslash \mathrm{H}, 0,-5.008219425,0.3160507674,-0.1821985514 \backslash \mathrm{H}, 0,-2.893019$ $2359,-0.833987415,-0.9185302055 \backslash \mathrm{N}, 0,-5.2405862511,2.9401991483,0.20741$ 
$35064 \backslash \mathrm{H}, 0,-5.272120156,3.940688324,0.3256950236 \backslash \mathrm{H}, 0,-6.1168228636,2.44$ $69902483,0.2775428093 \backslash \backslash$ Version=EM64L-G09RevB.01 \State=1-A \MP2/GTBas $1=-$ $285.6911966 \backslash \mathrm{MP} 4 / \mathrm{GTBas} 1=-285.7796412 \backslash \mathrm{CCSD}(\mathrm{T}) / \mathrm{G} 3 \mathrm{Bas} 1=-285.7800367 \backslash \mathrm{MP} 2 / \mathrm{GT}$ Bas $2=-285.702478 \backslash \mathrm{MP} 4 / \mathrm{GTBas} 2=-285.7912808 \backslash \mathrm{MP} 2 / \mathrm{GTBas} 3=-285.8947142 \backslash \mathrm{MP} 4 / \mathrm{G}$ TBas $3=-285.9926679 \backslash \mathrm{HF} / \mathrm{GTLargeXP}=-284.8854198 \backslash \mathrm{MP} 2 / \mathrm{GTLargeXP}=-286.308410$ $2 \backslash \mathrm{HF} / \mathrm{GFHFB} 1=-284.9083523 \backslash \mathrm{HF} / \mathrm{GFHFB} 2=-284.9125594 \backslash \mathrm{G} 4=-286.4524827 \backslash \mathrm{FreqCo}$ ord $=-5.4675242415,5.7576679525,-0.5278423915,-7.7581364567,4.363688449$ $9,-0.2475417378,-3.218681613,4.5469350639,-1.2028887636,-3.5827470041$, $2.0263943802,-0.9898996925,-7.7626429015,1.6991816471,-0.6586785914,-5$ $.5474088067,0.4290211662,-1.3356426949,-5.4102036982,7.7659110901,-0.1$ $131970509,-1.4492883214,5.5285867345,-1.5067214068,-9.4641631266,0.597$ $2493945,-0.3443053639,-5.4670140531,-1.5760078127,-1.7357705333,-9.903$ $2727903,5.5561711665,0.3919547233,-9.9628632345,7.4468217074,0.6154743$ $975,-11.5591200156,4.624141419,0.5244798997 \backslash \mathrm{PG}=\mathrm{C} 01 \quad[\mathrm{X}(\mathrm{C} 6 \mathrm{H} 6 \mathrm{~N} 1)] \backslash \mathrm{NImag}=0$ 11

${ }^{1}$ m-cyanophenyl cation

$\backslash \backslash 1,1 \backslash C, 0,-2.9631357077,3.1231851354,-0.5155130535 \backslash C, 0,-3.9907271466,2$ $.2148040016,-0.9698565933 \backslash C, 0,-1.7925952337,2.6153442436,0.0658075322 \backslash$ $C, 0,-1.596637707,1.2491342472,0.2160834695 \backslash C, 0,-3.5290274704,1.0066649$ $949,-0.6989112567 \backslash \mathrm{C}, 0,-2.556149029,0.2643730128,-0.2025258666 \backslash \mathrm{H}, 0,-1.0$ $28741983,3.3055829138,0.4029270831 \backslash H, 0,-2.4269216662,-0.8036961581,-0$. $0965214257 \backslash \mathrm{H}, 0,-0.6930901365,0.844511507,0.6653277104 \backslash \mathrm{H}, 0,-4.919043164$ $2,2.5316960693,-1.4273258722 \backslash C, 0,-3.2015672136,4.5153973676,-0.6871927$ $394 \backslash \mathrm{N}, 0,-3.3952635421,5.6483126649,-0.8267389876 \backslash \backslash$ Version=EM64L-G0 9Rev B. 01 \State $=1-A \backslash M P 2 / G T B a s 1=-322.479414 \backslash \mathrm{MP} 4 / \mathrm{GTBas} 1=-322.5696449 \backslash \mathrm{CCSD}(\mathrm{T}) /$ G3Bas $1=-322.5626253 \backslash \mathrm{MP} 2 / \mathrm{GTBas} 2=-322.4900235 \backslash \mathrm{MP} 4 / \mathrm{GTBas} 2=-322.5806604 \backslash \mathrm{MP}$ $2 /$ GTBas $3=-322.6824398 \backslash \mathrm{MP} 4 / \mathrm{GTBas} 3=-322.7818204 \backslash \mathrm{HF} / \mathrm{GTL}$ LargeXP $=-321.556981$ $4 \backslash \mathrm{MP} 2 / \mathrm{GTL}$ argeXP $=-323.1458096 \backslash \mathrm{HF} / \mathrm{GFHFB} 1=-321.5821978 \backslash \mathrm{HF} / \mathrm{GFHFB} 2=-321.586$ $5227 \backslash \mathrm{G} 4=-323.312242 \backslash$ FreqCoord $=-5.5995149822,5.9019645681,-0.974178489$, $-7.5413813781,4.185373001,-1.8327633495,-3.3875140587,4.9422843637,0.1$ $243582134,-3.0172079996,2.3605216304,0.4083385792,-6.6688954344,1.9023$ $21148,-1.3207508664,-4.8304216197,0.4995925912,-0.3827184228,-1.944040$ $6093,6.2466464167,0.7614218385,-4.5862172951,-1.5187656329,-0.18239906$ $06,-1.3097505434,1.5958954643,1.2572871612,-9.2956444163,4.7842122226$, $-2.6972550008,-6.0500852298,8.5328644059,-1.2986060781,-6.4161182436,1$ $0.6737640495,-1.56231027 \backslash P G=C 01 \quad[\mathrm{X}(\mathrm{C} 7 \mathrm{H} 4 \mathrm{~N} 1)] \backslash \mathrm{NImag}=0 \backslash \backslash$

${ }^{1}$ o-cyanophenyl cation

$\backslash \backslash 1,1 \backslash C, 0,-2.7855987682,2.9287826021,-0.3294817141 \backslash \mathrm{C}, 0,-3.765687142,2$. $0195843445,-0.2830223207 \backslash C, 0,-1.6073538728,2.1226740888,-0.5421113148 \backslash$ $C, 0,-1.6674307683,0.7396672405,-0.6488310974 \backslash C, 0,-4.1178837345,0.75295$ $49332,-0.3439167908 \backslash \mathrm{C}, 0,-2.8761456282,0.0576052879,-0.5552741281 \backslash \mathrm{C}, 0,-$ $2.7978087127,4.336370724,-0.2117683992 \backslash \mathrm{H}, 0,-0.6800724809,2.6866510996$, $-0.6105221568 \backslash \mathrm{H}, 0,-5.0830082715,0.2698885681,-0.2641353151 \backslash \mathrm{H}, 0,-2.9550$ $925545,-1.0233165126,-0.6347178426 \backslash \mathrm{H}, 0,-0.7518168424,0.1820321445,-0.8$ $083860874 \backslash \mathrm{N}, 0,-2.7943912239,5.4886054795,-0.117072833 \backslash \backslash$ Version=EM64L-G 0 9RevB.01 \State=1-A \MP2/GTBas $1=-322.4717914 \backslash \mathrm{MP} 4 / \mathrm{GTBas} 1=-322.5621758 \backslash \mathrm{CC}$ $\mathrm{SD}(\mathrm{T}) / \mathrm{GBBas} 1=-322.5551897 \backslash \mathrm{MP} 2 / \mathrm{GTBas} 2=-322.482275 \backslash \mathrm{MP} 4 / \mathrm{GTBas} 2=-322.57307$ $17 \backslash \mathrm{MP} 2 / \mathrm{GTBas} 3=-322.6755976 \backslash \mathrm{MP} 4 / \mathrm{GTBas} 3=-322.7751679 \backslash \mathrm{HF} / \mathrm{GTL}$ argeXP $=-321.5$ $502268 \backslash \mathrm{MP} 2 / \mathrm{GTL}$ argeXP $=-323.1385338 \backslash \mathrm{HF} / \mathrm{GFHFB} 1=-321.5755511 \backslash \mathrm{HF} / \mathrm{GFHFB} 2=-32$ $1.5798885 \backslash \mathrm{G} 4=-323.3050985 \backslash$ FreqCoord $=-5.264018788,5.5345970207,-0.62263$ $02054,-7.1161174005,3.8164613134,-0.5348346757,-3.0374586182,4.0112726$ $972,-1.0244419185,-3.1509874977,1.397768514,-1.2261130806,-7.781672505$ $2,1.4228786141,-0.6499085471,-5.4351275557,0.1088582179,-1.0493160307$, $-5.2870922392,8.194553079,-0.4001842781,-1.2851507394,5.0770347929,-1$. $1537196744,-9.6054935643,0.5100154801,-0.4991434076,-5.5843156253,-1.9$ 
$337879561,-1.1994428942,-1.4207279343,0.3439909005,-1.5276283149,-5.28$ $06341213,10.3719612076,-0.2212355919 \backslash \mathrm{PG}=\mathrm{C} 01 \quad[\mathrm{X}(\mathrm{C} 7 \mathrm{H} 4 \mathrm{~N} 1)] \backslash \mathrm{NImag}=0 \backslash \backslash$

${ }^{1} \mathrm{p}$-cyanophenyl cation

$\backslash \backslash 1,1 \backslash \mathrm{C}, 0,-2.7815548718,2.9588453246,0 . \backslash \mathrm{C}, 0,-3.9758042457,2.2226497767$ $, 0 . \backslash \mathrm{C}, 0,-1.5057198316,2.3172756802,0 . \backslash \mathrm{C}, 0,-1.8030474712,1.0286119624,0$ $. \backslash \mathrm{C}, 0,-3.9570428613,0.8198441191,0 . \backslash \mathrm{C}, 0,-2.731490144,0.0867768359,0 . \backslash \mathrm{C}$ $, 0,-5.2288700012,2.9112719939,0 . \backslash \mathrm{H}, 0,-2.7754714043,4.0453264112,0 . \backslash \mathrm{H}, 0$ $,-0.5573238145,2.8378632771,0 . \backslash \mathrm{H}, 0,-4.8709450074,0.2322544714,0 . \backslash \mathrm{H}, 0,-$ $2.6625208488,-0.9929037741,0 . \backslash \mathrm{N}, 0,-6.2418995138,3.4679838932,0.1 \backslash$ Versi on=EM6 4L-G09RevB. $01 \backslash$ State $=1-A^{\prime} \backslash M P 2 / G T B a s 1=-322.4799915 \backslash M P 4 / G T B a s 1=-322$ $.5699298 \backslash \mathrm{CCSD}(\mathrm{T}) / \mathrm{G} 3 \mathrm{Bas} 1=-322.5632872 \backslash \mathrm{MP} 2 / \mathrm{GTB} a \mathrm{~s} 2=-322.4907267 \backslash \mathrm{MP} 4 / \mathrm{GTBas}$ $2=-322.5810666 \backslash \mathrm{MP} 2 / \mathrm{GTBas} 3=-322.6831941 \backslash \mathrm{MP} 4 / \mathrm{GTBas} 3=-322.7822815 \backslash \mathrm{HF} / \mathrm{GTLa}$ rgeXP $=-321.5597671 \backslash \mathrm{MP} 2 / \mathrm{GTLargeXP}=-323.1467824 \backslash \mathrm{HF} / \mathrm{GFHFB} 1=-321.5849516 \backslash \mathrm{H}$ $\mathrm{F} / \mathrm{GFHFB} 2=-321.5892778 \backslash \mathrm{G} 4=-323.3133451 \backslash$ FreqCoord $=-5.2563769313,5.591407$ $333,0 .,-7.5131811823,4.2001993673,0 .,-2.8453981146,4.3790164099,0 .,-3$. $4072659251,1.943794906,0 .,-7.4777273039,1.5492808567,0 .,-5.1617683069$, $0.1639844545,0 .,-9.8811322868,5.5015067669,0 .,-5.2448808437,7.64455903$ $52,0 .,-1.0531893768,5.3627843963,0 .,-9.2047520723,0.4388973441,0 .,-5.0$ $314352273,-1.8763162094,0 .,-11.7954806302,6.5535397915,0 . \backslash P G=C S \quad[S G(C 7$ H4N1) ] \NImag=0 \

${ }^{1}$ m-bromophenyl cation

$\backslash \backslash 1,1 \backslash \mathrm{C}, 0,-2.9808830711,2.8769923246,-0.3969639881 \backslash \mathrm{C}, 0,-3.9803078081$, $1.9560047394,-0.8684069751 \backslash \mathrm{C}, 0,-1.784970942,2.3994157975,0.1511902439 \backslash$ $\mathrm{C}, 0,-1.5386718601,1.0376636662,0.2513058293 \backslash \mathrm{C}, 0,-3.4682301857,0.755795$ $0658,-0.6437633677 \backslash \mathrm{C}, 0,-2.4649570022,0.0298713575,-0.187196843 \backslash \mathrm{H}, 0,-1$. $0416872308,3.1039367858,0.5020063993 \backslash \mathrm{H}, 0,-2.2968483197,-1.0347554104,-$ $0.1202295036 \backslash \mathrm{H}, 0,-0.6133758605,0.6550572172,0.6752901222 \backslash \mathrm{H}, 0,-4.927215$ $5549,2.2431803876,-1.3033498517 \backslash \mathrm{Br}, 0,-3.3501334487,4.7018480407,-0.549$ $2492889 \backslash \backslash$ Version=EM64L-G09RevB.01 \State=1-A \MP2/GTBas $1=-2802.2697261 \backslash \mathrm{M}$ $\mathrm{P} 4 / \mathrm{GTBas} 1=-2802.345557 \backslash \mathrm{CCSD}(\mathrm{T}) / \mathrm{G} 3 \mathrm{Bas} 1=-2802.3399081 \backslash \mathrm{MP} 2 / \mathrm{GTBas} 2=-2802.2$ $786824 \backslash \mathrm{MP} 4 / \mathrm{GTBas} 2=-2802.3548093 \backslash \mathrm{MP} 2 / \mathrm{GTBas} 3=-2802.4642892 \backslash \mathrm{MP} 4 / \mathrm{GTB}$ s $3=-2$ $802.5534939 \backslash \mathrm{HF} / \mathrm{GTLargeXP}=-2801.6305824 \backslash \mathrm{MP} 2 / \mathrm{GTLargeXP}=-2803.9261651 \backslash \mathrm{HF} /$ $\mathrm{GFHFB} 1=-2801.713218 \backslash \mathrm{HF} / \mathrm{GFHFB} 2=-2801.7167115 \backslash \mathrm{G} 4=-2804.141581 \backslash \mathrm{FreqCoord}=$ $-5.6330526385,5.4367275798,-0.7501532222,-7.521691682,3.6963132721,-1$. $6410513549,-3.3731062355,4.5342387362,0.2857081548,-2.907668424,1.9609$ $001471,0.4748991929,-6.554005217,1.4282456869,-1.2165364587,-4.6580936$ $635,0.056448685,-0.3537507662,-1.9685035823,5.865590459,0.9486546116,-$ $4.3404142931,-1.9554043402,-0.2272008349,-1.1591123929,1.2378787418,1$. $2761133912,-9.3110879965,4.2389965993,-2.462974275,-6.3308347267,8.885$ $2051155,-1.0379307347 \backslash \mathrm{PG}=\mathrm{C} 01 \quad[\mathrm{X}(\mathrm{C} 6 \mathrm{H} 4 \mathrm{Br} 1)] \backslash \mathrm{NImag}=0 \backslash \backslash$

${ }^{1}$ o-bromophenyl cation

$\backslash \backslash 1,1 \backslash \mathrm{C}, 0,-2.7237930422,2.6903726842,-0.2928510296 \backslash \mathrm{C}, 0,-3.7101564397$, $1.800903546,-0.2104444247 \backslash \mathrm{C}, 0,-1.5433822845,1.877620811,-0.2500359178 \backslash$ $\mathrm{C}, 0,-1.6103999499,0.4919301015,-0.1445725736 \backslash \mathrm{C}, 0,-4.0747229032,0.53547$ $18773,-0.1094096773 \backslash \mathrm{C}, 0,-2.8296099234,-0.1766376468,-0.0751679941 \backslash \mathrm{H}, 0$, $-0.6020495165,2.4199017083,-0.3055875775 \backslash \mathrm{H}, 0,-5.0496951634,0.070583595$ $2,-0.059170704 \backslash \mathrm{H}, 0,-2.9084151506,-1.2566775091,0.0074465726 \backslash \mathrm{H}, 0,-0.689$ $3423708,-0.0782395539,-0.1159804755 \backslash \mathrm{Br}, 0,-2.791023266,4.538320337,-0.4$ $310468511 \backslash \backslash$ Version=EM64L-G0 9RevB.01 \State=1-A \MP2/GTBas $1=-2802.2632306$ $\backslash \mathrm{MP} 4 / \mathrm{GTBas} 1=-2802.3393712 \backslash \mathrm{CCSD}(\mathrm{T}) / \mathrm{G} 3 \mathrm{Bas} 1=-2802.3342321 \backslash \mathrm{MP} 2 / \mathrm{GTB}$ s $2=-280$ $2.2723892 \backslash \mathrm{MP} 4 / \mathrm{GTBas} 2=-2802.3488382 \backslash \mathrm{MP} 2 / \mathrm{GTB} a \mathrm{~s} 3=-2802.4573783 \backslash \mathrm{MP} 4 / \mathrm{GTB} a \mathrm{~s} 3$ $=-2802.5468802 \backslash \mathrm{HF} / \mathrm{GTL}$ argeXP $=-2801.6269143 \backslash \mathrm{MP} 2 / \mathrm{GTL}$ argeXP $=-2803.919521 \backslash \mathrm{H}$ $\mathrm{F} / \mathrm{GFHFB} 1=-2801.7095186 \backslash \mathrm{HF} / \mathrm{GFHFB} 2=-2801.7130423 \backslash \mathrm{G} 4=-2804.1354512 \backslash \mathrm{FreqCo}$ ord $=-5.1472228924,5.0840675686,-0.5534082438,-7.0111795812,3.403214493$ 
$8,-0.397682329,-2.9165698361,3.5481891141,-0.4724994079,-3.0432148698$, $0.9296131684,-0.2732025704,-7.7001103545,1.0118952,-0.2067543264,-5.34$ $71878181,-0.3337967772,-0.1420469228,-1.1377087046,4.5729514973,-0.577$ $4768311,-9.5425409133,0.1333836643,-0.1118164256,-5.4961081153,-2.3747$ $763295,0.0140719828,-1.3026682926,-0.1478513297,-0.2191713355,-5.27426$ $96032,8.5761825403,-0.814560499 \backslash \mathrm{PG}=\mathrm{C} 01 \quad[\mathrm{X}(\mathrm{C} 6 \mathrm{H} 4 \mathrm{Br} 1)] \backslash \mathrm{NImag}=0 \backslash \backslash$

${ }^{1}$ p-bromophenyl cation

$\backslash \backslash 1,1 \backslash \mathrm{C}, 0,-2.8490491285,3.003181546,-0.2320604064 \backslash \mathrm{C}, 0,-4.0254039408,2$ $.268440059,-0.0592802913 \backslash C, 0,-1.6320291737,2.3806037471,-0.6530662553 \backslash$ $C, 0,-1.9556642739,1.1060731347,-0.7934185018 \backslash C, 0,-4.0568627138,0.89073$ $40727,-0.2925924721 \backslash \mathrm{C}, 0,-2.8925558179,0.1759471451,-0.716242337 \backslash \mathrm{H}, 0,-2$ $.8015401884,4.0736985591,-0.0566823825 \backslash \mathrm{H}, 0,-0.6986482994,2.9061863368$, $-0.8002139429 \backslash \mathrm{H}, 0,-4.9590683799,0.3002092937,-0.1648125291 \backslash \mathrm{H}, 0,-2.8678$ $586021,-0.8877030868,-0.9089233989 \backslash \mathrm{Br}, 0,-5.5902895519,3.1472692723,0.4$ $957821927 \backslash \backslash$ Version=EM64L-G0 9RevB.01 \State $=1-A \backslash M P 2 / G T B a s 1=-2802.2671975$ $\backslash \mathrm{MP} 4 / \mathrm{GTBas} 1=-2802.342764 \backslash \mathrm{CCSD}(\mathrm{T}) / \mathrm{G} 3 \mathrm{Bas} 1=-2802.3376972 \backslash \mathrm{MP} 2 / \mathrm{GTBas} 2=-2802$ $.2760938 \backslash \mathrm{MP} 4 / \mathrm{GTBas} 2=-2802.3519599 \backslash \mathrm{MP} 2 / \mathrm{GTBas} 3=-2802.4614768 \backslash \mathrm{MP} 4 / \mathrm{GTBas} 3=$ $-2802.5504117 \backslash \mathrm{HF} / \mathrm{GTLargeXP}=-2801.6297241 \backslash \mathrm{MP} 2 / \mathrm{GTLargeXP}=-2803.9232568 \backslash \mathrm{H}$ $\mathrm{F} / \mathrm{GFHFB} 1=-2801.7123328 \backslash \mathrm{HF} / \mathrm{GFHFB} 2=-2801.7158285 \backslash \mathrm{G} 4=-2804.1392072 \backslash \mathrm{FreqCo}$ ord $=-5.3839225919,5.6751906493,-0.4385306144,-7.6069110224,4.286730460$ $4,-0.1120235156,-3.0840881793,4.4986891129,-1.2341163691,-3.6956698856$ $, 2.0901753076,-1.4993436772,-7.6663594879,1.6832434546,-0.5529196408,-$ $5.4661383198,0.3324919181,-1.3535018617,-5.2941437064,7.6981746247,-0$. $1071141795,-1.320253949,5.4918962678,-1.5121851998,-9.3712811122,0.567$ $3133477,-0.3114505432,-5.4194673459,-1.6775157213,-1.7176162998,-10.56$ $41162567,5.9474769911,0.9368925658 \backslash \mathrm{PG}=\mathrm{C} 01 \quad[\mathrm{X}(\mathrm{C} 6 \mathrm{H} 4 \mathrm{Br} 1)] \backslash \mathrm{NImag}=0 \backslash \backslash$

${ }^{1}$ m-chlorophenyl cation

$\backslash \backslash 1,1 \backslash \mathrm{C}, 0,-2.9837300757,2.8963031481,-0.3980943592 \backslash \mathrm{C}, 0,-3.9775857843$, $1.9704549166,-0.8669838942 \backslash C, 0,-1.7875440155,2.4156707606,0.1501639981$ $\backslash \mathrm{C}, 0,-1.5483919864,1.0536061985,0.2469762386 \backslash \mathrm{C}, 0,-3.4695485046,0.76736$ $77704,-0.6442520308 \backslash \mathrm{C}, 0,-2.4706060454,0.0373606116,-0.1897502731 \backslash \mathrm{H}, 0,-$ $1.0436421741,3.1194879393,0.5012642775 \backslash \mathrm{H}, 0,-2.295965148,-1.0257635256$, $-0.1197352974 \backslash \mathrm{H}, 0,-0.6238917828,0.6680982921,0.6705808763 \backslash \mathrm{H}, 0,-4.92459$ $87188,2.2594740756,-1.3019595625 \backslash \mathrm{Cl}, 0,-3.3218162287,4.5629498027,-0.53$ $7568969 \backslash \backslash$ Version=EM64L-G09RevB.01 \State=1-A \MP2/GTBas1=-689.5131519 $\backslash \mathrm{MP}$ $4 / \mathrm{GTBas} 1=-689.6001337 \backslash \mathrm{CCSD}(\mathrm{T}) / \mathrm{G} 3 \mathrm{Bas} 1=-689.5965857 \backslash \mathrm{MP} 2 / \mathrm{GTBas} 2=-689.5219$ $166 \backslash \mathrm{MP} 4 / \mathrm{GTBas} 2=-689.6092785 \backslash \mathrm{MP} 2 / \mathrm{GTBas} 3=-689.7198834 \backslash \mathrm{MP} 4 / \mathrm{GTBas} 3=-689.82$ $12301 \backslash \mathrm{HF} / \mathrm{GTL}$ argeXP $=-688.7435155 \backslash \mathrm{MP} 2 / \mathrm{GTL}$ argeXP $=-690.4165595 \backslash \mathrm{HF} / \mathrm{GFHFB} 1=-$ $688.7713927 \backslash \mathrm{HF} / \mathrm{GFHFB} 2=-688.775863 \backslash \mathrm{G} 4=-690.5922679 \backslash$ FreqCoord $=-5.6384326$ $975,5.4732197478,-0.752289314,-7.5165478023,3.7236201496,-1.6383621217$ $,-3.3779686399,4.5649561647,0.2837688314,-2.9260368007,1.9910271671,0$. $4667174524,-6.5564964785,1.4501149292,-1.2174598988,-4.6687688081,0.07$ $0601324,-0.3585760499,-1.9721978898,5.8949778801,0.9472522047,-4.33874$ $53404,-1.9384121405,-0.2262669206,-1.1789846061,1.2625228019,1.2672142$ $062,-9.306142893,4.2697872073,-2.4603470091,-6.277322936,8.6227254852$, $-1.0158581289 \backslash \mathrm{PG}=\mathrm{C} 01 \quad[\mathrm{X}(\mathrm{C} 6 \mathrm{H} 4 \mathrm{Cl} 1)] \backslash \mathrm{NImag}=0 \backslash \backslash$

${ }^{1}$ o-chlorophenyl cation

$\backslash \backslash 1,1 \backslash \mathrm{C}, 0,-2.7299537438,2.71096221,-0.2943058314 \backslash \mathrm{C}, 0,-3.7188198953,1$. $8151040643,-0.2113774564 \backslash C, 0,-1.55403686,1.8905555429,-0.2508437547 \backslash C$, $0,-1.6128249098,0.5058260196,-0.145583641 \backslash C, 0,-4.0714640785,0.54666634$ $9,-0.1103007905 \backslash \mathrm{C}, 0,-2.8286057874,-0.1678941234,-0.0758439131 \backslash \mathrm{H}, 0,-0.6$ $170446958,2.4416092218,-0.3069866929 \backslash \mathrm{H}, 0,-5.0465746096,0.080764092,-0$. $0599853143 \backslash \mathrm{H}, 0,-2.9069014483,-1.247804582,0.0067490542 \backslash \mathrm{H}, 0,-0.68818621$ $75,-0.0585470498,-0.1174862385 \backslash \mathrm{Cl}, 0,-2.7581777478,4.3963182859,-0.4208$ 
$550192 \backslash \backslash$ Version=EM64L-G0 9RevB.01 \State=1-A \MP $2 /$ GTBas $1=-689.5037357 \backslash M P 4$ $/ \mathrm{GTBas} 1=-689.5911706 \backslash \mathrm{CCSD}(\mathrm{T}) / \mathrm{G} 3 \mathrm{Bas} 1=-689.5880292 \backslash \mathrm{MP} 2 / \mathrm{GTBas} 2=-689.51243$ $02 \backslash \mathrm{MP} 4 / \mathrm{GTBas} 2=-689.6002515 \backslash \mathrm{MP} 2 / \mathrm{GTBas} 3=-689.7095837 \backslash \mathrm{MP} 4 / \mathrm{GTBas} 3=-689.811$ $4853 \backslash \mathrm{HF} / \mathrm{GTLargeXP}=-688.7361924 \backslash \mathrm{MP} 2 / \mathrm{GTL}$ argeXP $=-690.4065312 \backslash \mathrm{HF} / \mathrm{GFHFB} 1=-6$ $88.7640215 \backslash \mathrm{HF} / \mathrm{GFHFB} 2=-688.768557 \backslash \mathrm{G} 4=-690.5830335 \backslash \mathrm{FreqCoord}=-5.15886493$ $11,5.1229761336,-0.5561574207,-7.0275511396,3.4300495842,-0.3994455032$ $,-2.9367040658,3.5726322151,-0.4740259986,-3.0477973798,0.9558726479,-$ $0.2751132109,-7.6939520683,1.0330496856,-0.2084382864,-5.345290276,-0$. $3172739126,-0.1433242246,-1.1660454867,4.6139727528,-0.5801207759,-9.5$ $366439213,0.1526220153,-0.113355816,-5.4932476325,-2.3580089274,0.0127$ $538641,-1.3004834796,-0.11063789,-0.2220168152,-5.2122005692,8.3078375$ $533,-0.7953007279 \backslash \mathrm{PG}=\mathrm{C} 01 \quad[\mathrm{X}(\mathrm{C} 6 \mathrm{H} 4 \mathrm{Cl} 1)] \backslash \mathrm{NImag}=0 \backslash \backslash$

${ }^{1}$ p-chlorophenyl cation

$\backslash \backslash 1,1 \backslash \mathrm{C}, 0,-2.8586224968,3.0113284086,-0.2281797026 \backslash \mathrm{C}, 0,-4.0352168418$, $2.273947849,-0.0557992479 \backslash C, 0,-1.6439228849,2.3885315994,-0.6486308536$ $\backslash \mathrm{C}, 0,-1.9696192161,1.113912305,-0.7884633059 \backslash \mathrm{C}, 0,-4.0688394378,0.89467$ $86351,-0.288829974 \backslash \mathrm{C}, 0,-2.905534641,0.1819910301,-0.7118582154 \backslash \mathrm{H}, 0,-2$. $8187848864,4.0818337509,-0.0508367334 \backslash \mathrm{H}, 0,-0.7089217273,2.911230555,-0$ $.7967030314 \backslash \mathrm{H}, 0,-4.9749783674,0.3106851311,-0.1588946522 \backslash \mathrm{H}, 0,-2.877490$ $2988,-0.8815542698,-0.9053813678 \backslash \mathrm{Cl}, 0,-5.4670392315,3.0780544675,0.452$ $0678521 \backslash \backslash$ Version=EM64L-G09RevB.01 \State=1-A \MP2/GTBas1=-689.5108675 \MP $4 / \mathrm{GTBas} 1=-689.5976628 \backslash \mathrm{CCSD}(\mathrm{T}) / \mathrm{G} 3 \mathrm{Bas} 1=-689.5945752 \backslash \mathrm{MP} 2 / \mathrm{GTBas} 2=-689.5195$ $185 \backslash \mathrm{MP} 4 / \mathrm{GTBas} 2=-689.6066935 \backslash \mathrm{MP} 2 / \mathrm{GTBas} 3=-689.7173936 \backslash \mathrm{MP} 4 / \mathrm{GTBas} 3=-689.81$ $8554 \backslash \mathrm{HF} / \mathrm{GTLargeXP}=-688.7432228 \backslash \mathrm{MP} 2 / \mathrm{GTLargeXP}=-690.4139873 \backslash \mathrm{HF} / \mathrm{GFHFB} 1=-6$ $88.7711273 \backslash \mathrm{HF} / \mathrm{GFHFB} 2=-688.775574 \backslash \mathrm{G} 4=-690.590061 \backslash$ FreqCoord $=-5.402013636$ $2,5.6905859884,-0.431197147,-7.6254547178,4.297138675,-0.105445297,-3$. $106564036,4.5136705827,-1.2257346746,-3.7220409045,2.1049891926,-1.489$ $9797141,-7.6889922161,1.6906975974,-0.5458095498,-5.4906647411,0.34391$ $32056,-1.3452170725,-5.3267314628,7.7135479092,-0.0960675036,-1.339667$ $9143,5.5014284587,-1.5055505386,-9.4013466315,0.5871098113,-0.30026737$ $67,-5.4376686148,-1.6658961413,-1.710922831,-10.3312069052,5.816679965$ $7,0.854284434 \backslash \mathrm{PG}=\mathrm{C} 01 \quad[\mathrm{X}(\mathrm{C} 6 \mathrm{H} 4 \mathrm{Cl} 1)] \backslash \mathrm{NImag}=0 \backslash \backslash$

${ }^{1}$ m-ethynylphenyl cation 11

$1,1 \backslash C, 0,-2.9582626013,2.9502879719,-0.3859814777 \backslash C, 0,-3.9694427312,2.0$ $213123192,-0.8732320638 \backslash \mathrm{C}, 0,-1.7748120458,2.4271091225,0.1645339197 \backslash \mathrm{C}$, $0,-1.5498519188,1.0625301929,0.2536309261 \backslash C, 0,-3.4832608609,0.81432683$ $08,-0.6592375888 \backslash \mathrm{C}, 0,-2.4879249094,0.0745031318,-0.1999245608 \backslash \mathrm{H}, 0,-1.0$ $221151459,3.1156563912,0.5272133615 \backslash \mathrm{H}, 0,-2.3360259032,-0.9929088012,-0$ $.141502768 \backslash \mathrm{H}, 0,-0.6338911151,0.6621369538,0.6797910894 \backslash \mathrm{H}, 0,-4.90508235$ $74,2.3388512035,-1.3096676062 \backslash \mathrm{C}, 0,-3.2343075702,4.3245689102,-0.499073$ $5513 \backslash \mathrm{C}, 0,-3.4779406327,5.5009736005,-0.5993544697 \backslash \mathrm{H}, 0,-3.678632208,6.5$ $451421728,-0.6812252105 \backslash \backslash$ Version=EM6 4L-G0 9RevB.01 \State=1-A \MP2 /GTBas 1 $=-306.4117088 \backslash \mathrm{MP} 4 / \mathrm{GTBas} 1=-306.5058688 \backslash \mathrm{CCSD}(\mathrm{T}) / \mathrm{G} 3 \mathrm{Bas} 1=-306.5006575 \backslash \mathrm{MP} 2 /$ GTBas2 $=-306.4221672 \backslash \mathrm{MP} 4 / \mathrm{GTBas} 2=-306.5165187 \backslash \mathrm{MP} 2 / \mathrm{GTBas} 3=-306.6171559 \backslash \mathrm{MP}$ $4 /$ GTBas $3=-306.7199428 \backslash \mathrm{HF} / \mathrm{GTLargeXP}=-305.5177565 \backslash \mathrm{MP} 2 / \mathrm{GTL}$ LargeXP $=-307.070$ $4437 \backslash \mathrm{HF} / \mathrm{GFHFB} 1=-305.5420313 \backslash \mathrm{HF} / \mathrm{GFHFB} 2=-305.5462064 \backslash \mathrm{G} 4=-307.230634 \backslash \mathrm{Freq}$ Coord $=-5.5903061456,5.5752362801,-0.7293992851,-7.5011596622,3.8197267$ $124,-1.650169451,-3.3539087038,4.5865715362,0.3109240478,-2.9287956731$ $, 2.0078910724,0.4792929892,-6.5824090766,1.5388546929,-1.2457784993,-4$ $.7014967179,0.1407905151,-0.3778026672,-1.931517702,5.8877373035,0.996$ $2888669,-4.4144491964,-1.8763257092,-0.2674014785,-1.1978806057,1.2512$ $575052,1.2846189865,-9.2692623148,4.4197882403,-2.4749131008,-6.111955$ $5372,8.1722508831,-0.9431123322,-6.5723553021,10.3953335692,-1.1326158$ $042,-6.9516074167,12.3685262074,-1.2873290826 \backslash \mathrm{PG}=\mathrm{C} 01 \quad[\mathrm{X}(\mathrm{C} 8 \mathrm{H} 5)] \backslash \mathrm{NImag}=0$ 
${ }^{1}$ o-ethynylphenyl cation

$\backslash 1$

$1,1 \backslash C, 0,-2.7416361302,2.800664121,-0.2615220457 \backslash C, 0,-3.7063729273,1.88$ $12572808,-0.1931276284 \backslash C, 0,-1.5635803327,1.9396434943,-0.2357010444 \backslash C$, $0,-1.6104732208,0.5580743571,-0.1576114126 \backslash C, 0,-4.0514009958,0.6062427$ $624,-0.1160824621 \backslash \mathrm{C}, 0,-2.8244059447,-0.123850166,-0.0970450146 \backslash \mathrm{H}, 0,-0$. $6329243313,2.4992497809,-0.2842271793 \backslash \mathrm{H}, 0,-5.0351366834,0.1578633494,-$ $0.0733538131 \backslash \mathrm{H}, 0,-2.8991843388,-1.2047099133,-0.0352617124 \backslash \mathrm{H}, 0,-0.6805$ $300829,0.001919341,-0.1436878064 \backslash \mathrm{C}, 0,-2.7120318623,4.1961245865,-0.340$ $1461552 \backslash \mathrm{C}, 0,-2.66035861,5.3953352516,-0.4081485215 \backslash \mathrm{H}, 0,-2.6215845398,6$ $.4595457542,-0.4687452042 \backslash \backslash$ Version=EM64L-G09RevB.01 $\backslash$ State=1-A \MP2/GTBa $\mathrm{s} 1=-306.4069782 \backslash \mathrm{MP} 4 / \mathrm{GTBas} 1=-306.5010835 \backslash \mathrm{CCSD}(\mathrm{T}) / \mathrm{G} 3 \mathrm{Bas} 1=-306.4962393 \backslash \mathrm{MP}$ $2 / \mathrm{GTBas} 2=-306.4174107 \backslash \mathrm{MP} 4 / \mathrm{GTBas} 2=-306.511714 \backslash \mathrm{MP} 2 / \mathrm{GTBas} 3=-306.613306 \backslash \mathrm{MP}$ $4 /$ GTBas $3=-306.7160481 \backslash \mathrm{HF} / \mathrm{GTL}$ LargeXP $=-305.5154254 \backslash \mathrm{MP} 2 / \mathrm{GTL}$ LargeXP $=-307.066$ $1517 \backslash \mathrm{HF} / \mathrm{GFHFB} 1=-305.5397519 \backslash \mathrm{HF} / \mathrm{GFHFB} 2=-305.5439437 \backslash \mathrm{G} 4=-307.2262415 \backslash \mathrm{Fre}$ qCoord $=-5.1809414421,5.2924881789,-0.494205044,-7.004029779,3.55506104$ $62,-0.3649583263,-2.9547386156,3.6653949997,-0.4454104231,-3.043353331$ $7,1.0546076967,-0.2978424053,-7.6560383366,1.1456327909,-0.2193640622$, $-5.3373537236,-0.2340428952,-0.1833885002,-1.196053649,4.7228976236,-0$ $.5371115285,-9.5150293733,0.2983184968,-0.1386186176,-5.4786644091,-2$. $2765718057,-0.0666349795,-1.2860154818,0.0036270289,-0.2715306027,-5.1$ $249974835,7.929526288,-0.6427830785,-5.0273491881,10.1957060206,-0.771$ $2889272,-4.9540768143,12.2067724182,-0.885800062 \backslash \mathrm{PG}=\mathrm{C} 01 \quad[\mathrm{X}(\mathrm{C} 8 \mathrm{H} 5)] \backslash \mathrm{NIma}$ $g=0 \backslash \backslash$

${ }^{1}$ p-ethynylphenyl cation $\backslash 1$

$1,1 \backslash \mathrm{C}, 0,-2.9659475102,3.0498136165,-0.2109999581 \backslash \mathrm{C}, 0,-4.1527213784,2.3$ $100603432,-0.0524273345 \backslash C, 0,-1.734038784,2.4427071377,-0.599268975 \backslash C, 0$ $,-2.0407606176,1.163187434,-0.7351411705 \backslash C, 0,-4.1442417509,0.921238626$ $2,-0.2802510086 \backslash \mathrm{C}, 0,-2.9649781644,0.2190021559,-0.6715857586 \backslash \mathrm{H}, 0,-2.93$ $7472643,4.122239202,-0.0432426678 \backslash \mathrm{H}, 0,-0.8034122433,2.976491026,-0.731$ $1954744 \backslash \mathrm{H}, 0,-5.0418077189,0.3207541814,-0.1669238006 \backslash \mathrm{H}, 0,-2.918891269$, $-0.845165297,-0.8554891971 \backslash C, 0,-5.3514320678,2.9610632301,0.3351552765$ $\backslash \mathrm{C}, 0,-6.3679737098,3.5131595106,0.6638212042 \backslash \mathrm{H}, 0,-7.2687021427,4.00240$ $88333,0.9550288644 \backslash \backslash$ Version=EM64L-G0 9RevB.01 \State=1-A \MP $2 /$ GTBas $1=-306$ $.4088425 \backslash \mathrm{MP} 4 / \mathrm{GTBas} 1=-306.5026481 \backslash \mathrm{CCSD}(\mathrm{T}) / \mathrm{G} 3 \mathrm{Bas} 1=-306.4980903 \backslash \mathrm{MP} 2 / \mathrm{GTBas}$ $2=-306.4194836 \backslash \mathrm{MP} 4 / \mathrm{GTBas} 2=-306.5134858 \backslash \mathrm{MP} 2 / \mathrm{GTBas} 3=-306.6144282 \backslash \mathrm{MP} 4 / \mathrm{GTB}$ as $3=-306.7168497 \backslash \mathrm{HF} / \mathrm{GTL}$ LargeXP $=-305.5180231 \backslash \mathrm{MP} 2 / \mathrm{GTLargeXP}=-307.0681335 \backslash$ $\mathrm{HF} / \mathrm{GFHFB} 1=-305.5422388 \backslash \mathrm{HF} / \mathrm{GFHFB} 2=-305.546418 \backslash \mathrm{G} 4=-307.2287844 \backslash \mathrm{FreqCoord}$ $=-5.6048285187,5.7633124915,-0.3987321349,-7.8475061114,4.3653813992,-$ $0.0990733041,-3.2768584055,4.6160475131,-1.1324542427,-3.85647867,2.19$ $81056915,-1.3892154812,-7.8314819377,1.7408887065,-0.5295976547,-5.602$ $9967207,0.4138540972,-1.2691131585,-5.5510188182,7.7899031461,-0.08171$ $67995,-1.5182291117,5.6247528762,-1.3817591961,-9.5276358035,0.6061375$ $589,-0.3154402681,-5.5159051101,-1.5971309484,-1.6166402921,-10.112741$ $0268,5.595598567,0.6333516845,-12.033726333,6.6389093362,1.2544402772$, $-13.7358563912,7.5634565668,1.8047430027 \backslash \mathrm{PG}=\mathrm{C} 01 \quad[\mathrm{X}(\mathrm{C} 8 \mathrm{H} 5)] \backslash \mathrm{NImag}=0 \backslash \backslash$

${ }^{1} \mathrm{~m}$-(dimethylamino) phenyl cation

$\backslash \backslash 1,1 \backslash C, 0,-2.8994782992,2.6687675851,-0.4092869285 \backslash C, 0,-3.7901938369$, $1.673530085,-1.0447740059 \backslash C, 0,-1.7242175322,2.1629172925,0.2480792023 \backslash$ $\mathrm{C}, 0,-1.4854274078,0.8214757084,0.3941592438 \backslash \mathrm{C}, 0,-3.4114462556,0.462477$ $0772,-0.6943855036 \backslash \mathrm{C}, 0,-2.4926031381,-0.1584888788,0.0228101048 \backslash \mathrm{H}, 0,-1$ $.0395223285,2.865695004,0.7044802528 \backslash \mathrm{H}, 0,-2.5717379172,-1.1092108824,0$ 
$.534333662 \backslash \mathrm{H}, 0,-0.6504322936,0.4786379574,0.9974773276 \backslash \mathrm{N}, 0,-3.08470303$ $59,3.9583445864,-0.6788167675 \backslash \mathrm{C}, 0,-1.9909586934,4.9384169487,-0.694512$ $7936 \backslash \mathrm{C}, 0,-4.3967574051,4.4715682194,-1.0742856747 \backslash \mathrm{H}, 0,-1.9762257625,5$. $5043347289,0.2430954858 \backslash \mathrm{H}, 0,-2.157485781,5.6344446537,-1.5196970835 \backslash \mathrm{H}$, $0,-1.032981034,4.4437673474,-0.8444119776 \backslash \mathrm{H}, 0,-5.1884474474,3.84488315$ $79,-0.664844124 \backslash \mathrm{H}, 0,-4.4828274316,4.5177644501,-2.167134503 \backslash \mathrm{H}, 0,-4.507$ $2647205,5.4834505674,-0.6786287024 \backslash \mathrm{H}, 0,-4.3378196796,1.9468543917,-1.9$ $380872148 \backslash \backslash$ Version=EM64L-G09RevB.01\State=1-A \MP2/GTBas1=-364.0420206 $\mathrm{MP} 4 / \mathrm{GTBas} 1=-364.162643 \backslash \mathrm{CCSD}(\mathrm{T}) / \mathrm{G} 3 \mathrm{Bas} 1=-364.1615374 \backslash \mathrm{MP} 2 / \mathrm{GTB}$ as $2=-364.056$ $4041 \backslash \mathrm{MP} 4 / \mathrm{GTBas} 2=-364.1777879 \backslash \mathrm{MP} 2 / \mathrm{GTBas} 3=-364.3176889 \backslash \mathrm{MP} 4 / \mathrm{GTBas} 3=-364.4$ $51961 \backslash \mathrm{HF} / \mathrm{GTLargeXP}=-362.9727039 \backslash \mathrm{MP} 2 / \mathrm{GTL}$ argeXP $=-364.849918 \backslash \mathrm{HF} / \mathrm{GFHFB} 1=-3$ $63.0011483 \backslash \mathrm{HF} / \mathrm{GFHFB} 2=-363.0065094 \backslash \mathrm{G} 4=-365.0214722 \backslash \mathrm{FreqCoord}=-5.4792199$ $137,5.0432398481,-0.7734402047,-7.1624283422,3.1625135359,-1.974336741$ $8,-3.2582989294,4.0873213309,0.4688017515,-2.8070509909,1.5523641137,0$ $.7448530236,-6.4466991401,0.8739550186,-1.3121984324,-4.710337289,-0.2$ $995005761,0.0431048511,-1.9644125099,5.4153787379,1.3312747438,-4.8598$ $803491,-2.0961047914,1.0097442848,-1.229138903,0.9044946563,1.88495897$ $3,-5.8292439391,7.4801872079,-1.282777785,-3.7623666724,9.3322555631,-$ $1.3124389757,-8.3086673683,8.4500393193,-2.0301057136,-3.7345254679,10$ $.4016851813,0.4593838922,-4.0770572617,10.6475573065,-2.8718112928,-1$. $9520512547,8.3975032848,-1.595707381,-9.8047447305,7.2657761815,-1.256$ $3733153,-8.4713161468,8.5373375435,-4.0952907038,-8.5174959301,10.3622$ $198357,-1.2824223935,-8.1972912083,3.6790216209,-3.6624540575 \backslash P G=C 01$ [ $\mathrm{X}(\mathrm{C} 8 \mathrm{H} 10 \mathrm{~N} 1)] \backslash \mathrm{NImag}=0 \backslash \backslash$

${ }^{1}$ o- (dimethylamino) phenyl cation

$\backslash \backslash 1,1 \backslash \mathrm{C}, 0,-2.8300148145,2.7470645054,-0.2988074419 \backslash \mathrm{C}, 0,-3.8191920653$, $1.7708058876,-0.4639157749 \backslash \mathrm{C}, 0,-1.614260926,2.1911433055,0.2286722643 \backslash$ $\mathrm{C}, 0,-1.5332464949,0.828115979,0.3914443585 \backslash \mathrm{C}, 0,-3.7623703594,0.4397317$ $675,-0.6158367432 \backslash \mathrm{C}, 0,-2.5846434739,-0.0333614433,0.0252744772 \backslash \mathrm{H}, 0,-0$. $7328564377,2.8190554576,0.2948420402 \backslash \mathrm{H}, 0,-4.5929672998,-0.2205157759,-$ $0.8357218728 \backslash \mathrm{H}, 0,-2.5931205212,-1.0641267436,0.3697507507 \backslash \mathrm{H}, 0,-0.61585$ $16502,0.3893458385,0.768609594 \backslash \mathrm{N}, 0,-3.0212013855,4.0126406719,-0.68149$ $5924 \backslash \mathrm{C}, 0,-2.0602513933,5.0786613821,-0.4234985557 \backslash \mathrm{C}, 0,-4.1848556791,4$. $2672743587,-1.5104519012 \backslash \mathrm{H}, 0,-1.5777582042,4.9257022662,0.5425182797 \backslash \mathrm{H}$ $, 0,-2.6024479059,6.025999626,-0.4009514683 \backslash \mathrm{H}, 0,-1.3014915417,5.1336247$ $205,-1.2126385172 \backslash \mathrm{H}, 0,-4.9603794774,3.5385408222,-1.1889318105 \backslash \mathrm{H}, 0,-3$. $9932822869,4.0841311766,-2.5726454612 \backslash \mathrm{H}, 0,-4.5350080832,5.2903961975,-$ $1.3700062937 \backslash \backslash$ Version=EM64L-G09RevB.01 \State=1-A \MP2/GTBas $1=-364.02539$ $53 \backslash \mathrm{MP} 4 / \mathrm{GTBas} 1=-364.1468368 \backslash \mathrm{CCSD}(\mathrm{T}) / \mathrm{GBBas} 1=-364.148816 \backslash \mathrm{MP} 2 / \mathrm{GTBas} 2=-364$. $0396879 \backslash \mathrm{MP} 4 / \mathrm{GTBas} 2=-364.1619686 \backslash \mathrm{MP} 2 / \mathrm{GTBas} 3=-364.3006478 \backslash \mathrm{MP} 4 / \mathrm{GTB}$ s $3=-36$ $4.436014 \backslash \mathrm{HF} / \mathrm{GTLargeXP}=-362.9614244 \backslash \mathrm{MP} 2 / \mathrm{GTLargeXP}=-364.8323427 \backslash \mathrm{HF} / \mathrm{GFHFB}$ $1=-362.9900201 \backslash \mathrm{HF} / \mathrm{GFHFB} 2=-362.9953928 \backslash \mathrm{G} 4=-365.0089503 \backslash \mathrm{FreqCoord}=-5.347$ $9529515,5.1911995846,-0.5646642316,-7.2172270523,3.3463381621,-0.87667$ $37633,-3.0505110572,4.1406607653,0.4321279537,-2.8974159695,1.56491240$ $65,0.7397226339,-7.1098495897,0.8309726124,-1.1637627872,-4.8842683168$ $,-0.0630439912,0.0477618401,-1.3848979619,5.3272427684,0.5571707084,-8$ $.679450334,-0.4167144244,-1.5792854628,-4.9002876147,-2.0109081161,0.6$ $987276562,-1.1637909573,0.7357570058,1.4524616357,-5.7092432109,7.5827$ $919396,-1.287840657,-3.8933108983,9.5972791339,-0.8002962879,-7.908231$ $1391,8.0639798718,-2.8543404303,-2.9815309098,9.3082282952,1.025210970$ $7,-4.9179138173,11.38748897,-0.7576884676,-2.4594625781,9.7011447907,-$ $2.2915546958,-9.3737587275,6.6868730639,-2.2467555126,-7.5462098935,7$. $7178894146,-4.8615953587,-8.5699232876,9.9973999477,-2.5889366955 \backslash P G=C$ $01[\mathrm{X}(\mathrm{C} 8 \mathrm{H} 10 \mathrm{~N} 1)] \backslash \mathrm{NImag}=0 \backslash \backslash$ 
${ }^{1} \mathrm{p}-($ dimethylamino) phenyl cation

$\backslash \backslash 1,1 \backslash \mathrm{C}, 0,-2.8732964189,3.0064728231,-0.1647277133 \backslash \mathrm{C}, 0,-4.0996377295$, $2.269720566,-0.0604118841 \backslash \mathrm{C}, 0,-1.6681653498,2.3637731536,-0.4419628641$ $\backslash \mathrm{C}, 0,-1.9167038964,1.0932054693,-0.8527365034 \backslash \mathrm{C}, 0,-4.0627887564,0.8467$ $544752,-0.2387322414 \backslash \mathrm{C}, 0,-2.8680932978,0.1844391279,-0.5160556703 \backslash \mathrm{H}, 0$, $-2.8619450577,4.0862345412,-0.0955279314 \backslash \mathrm{H}, 0,-0.720260955,2.8847415878$ $,-0.4955449221 \backslash \mathrm{H}, 0,-4.9713932516,0.2591557701,-0.230462431 \backslash \mathrm{H}, 0,-2.8013$ $129271,-0.8908073449,-0.6261436184 \backslash \mathrm{N}, 0,-5.2578868875,2.8976292796,0.21$ $96847111 \backslash \mathrm{C}, 0,-5.2902949672,4.3399186959,0.4759133995 \backslash \mathrm{C}, 0,-6.4980365835$ , $2.140407538,0.4087135765 \backslash \mathrm{H}, 0,-6.6832604993,1.4870342925,-0.4497378998$ $\backslash \mathrm{H}, 0,-7.3287349813,2.8383797657,0.4880750184 \backslash \mathrm{H}, 0,-6.4624863478,1.53430$ $94199,1.3200067996 \backslash \mathrm{H}, 0,-4.8047290703,4.8851636787,-0.3394191731 \backslash \mathrm{H}, 0,-4$ $.7954983282,4.5906566834,1.4201247772 \backslash \mathrm{H}, 0,-6.3270946948,4.6651404769,0$ $.5298645702 \backslash \backslash$ Version=EM64L-G09RevB.01 \State=1-A \MP2/GTBas $1=-364.017545$ $\backslash \mathrm{MP} 4 / \mathrm{GTBas} 1=-364.139834 \backslash \mathrm{CCSD}(\mathrm{T}) / \mathrm{G} 3 \mathrm{Bas} 1=-364.1418454 \backslash \mathrm{MP} 2 / \mathrm{GTBas} 2=-364.03$ $16978 \backslash \mathrm{MP} 4 / \mathrm{GTBas} 2=-364.1548416 \backslash \mathrm{MP} 2 / \mathrm{GTBas} 3=-364.2929768 \backslash \mathrm{MP} 4 / \mathrm{GTBas} 3=-364$. $4293077 \backslash \mathrm{HF} / \mathrm{GTL}$ argeXP $=-362.9621176 \backslash \mathrm{MP} 2 / \mathrm{GTL}$ argeXP $=-364.8253852 \backslash \mathrm{HF} / \mathrm{GFHFB} 1$ $=-362.9907014 \backslash \mathrm{HF} / \mathrm{GFHFB} 2=-362.9960821 \backslash \mathrm{G} 4=-365.0028974 \backslash$ FreqCoord $=-5.4297$ $433303,5.6814102617,-0.3112902647,-7.7471925528,4.2891502679,-0.114161$ $9162,-3.1523756554,4.4668839006,-0.8351887741,-3.622045442,2.065858944$ $,-1.6114384549,-7.6775580854,1.60013406,-0.4511385553,-5.4199108565,0$. $3485394399,-0.9752038862,-5.4082923665,7.7218641976,-0.1805216283,-1.3$ $610959491,5.4513715652,-0.9364441893,-9.3945717444,0.4897334312,-0.435$ $5108785,-5.2937142447,-1.6833819191,-1.1832399587,-9.935966255,5.47572$ $57731,0.4151439395,-9.9972086501,8.2012577742,0.899345988,-12.27950954$ $43,4.0447840596,0.7723567263,-12.6295320185,2.8100875629,-0.8498814622$ $,-13.8493020152,5.3637604184,0.922328117,-12.2123293348,2.8994246067,2$ $.4944513448,-9.0796220855,9.2316214672,-0.6414092814,-9.0621785111,8.6$ $750839017,2.6836469035,-11.9564761901,8.8158378728,1.0012989252 \backslash P G=C 01$

$[\mathrm{X}(\mathrm{C} 8 \mathrm{H} 10 \mathrm{~N} 1)] \backslash \mathrm{NImag}=0 \backslash \backslash$

${ }^{1}$ syn-m-thiomethoxyphenyl cation

$\backslash$

$\backslash 1,1 \backslash \mathrm{C}, 0,-0.2703178765,1.2136560608,-0.1210086609 \backslash \mathrm{C}, 0,-1.588861379,1.2$ $557567248,-0.1043311574 \backslash \mathrm{C}, 0,-2.6882012185,0.5328315004,0.0393143575 \backslash \mathrm{C}$, $0,-2.26519144,-0.8445560275,0.0404742744 \backslash \mathrm{C}, 0,-0.925199557,-1.170986931$ $8,0.101029552 \backslash \mathrm{C}, 0,0.0957797321,-0.1886339927,0.0417081524 \backslash \mathrm{H}, 0,0.424642$ $9814,1.9553717935,-0.4889568963 \backslash \mathrm{H}, 0,-3.6769681248,0.8471980045,0.34642$ $35964 \backslash \mathrm{H}, 0,-3.0345795723,-1.5993295066,0.1728722733 \backslash \mathrm{H}, 0,-0.637313178,-2$ $.210090229,0.2108836937 \backslash S, 0,1.7283664688,-0.7073783442,-0.1817823457 \backslash \mathrm{C}$ $, 0,2.7235949245,0.7435116882,0.2659764542 \backslash \mathrm{H}, 0,2.422483414,1.1369080333$ $, 1.2372681763 \backslash \mathrm{H}, 0,3.7497020853,0.3736027096,0.3185238678 \backslash \mathrm{H}, 0,2.6715067$ $4,1.5083715169,-0.5131473378 \backslash \backslash$ Version=EM64L-G09RevB .01 \State =1-A \MP2/G $\mathrm{TBas} 1=-667.3013051 \backslash \mathrm{MP} 4 / \mathrm{GTBas} 1=-667.4092466 \backslash \mathrm{CCSD}(\mathrm{T}) / \mathrm{G} 3 \mathrm{Bas} 1=-667.4077094$ $\backslash \mathrm{MP} 2 / \mathrm{GTBas} 2=-667.3127235 \backslash \mathrm{MP} 4 / \mathrm{GTBas} 2=-667.421279 \backslash \mathrm{MP} 2 / \mathrm{GTBas} 3=-667.547661$ $4 \backslash \mathrm{MP} 4 / \mathrm{GTBas} 3=-667.6702294 \backslash \mathrm{HF} / \mathrm{GTLargeXP}=-666.4128323 \backslash \mathrm{MP} 2 / \mathrm{GTLargeXP}=-668$ $.2928946 \backslash \mathrm{HF} / \mathrm{GFHFB} 1=-666.4411729 \backslash \mathrm{HF} / \mathrm{GFHFB} 2=-666.4468793 \backslash \mathrm{G} 4=-668.4761935$ $\backslash$ FreqCoord $=-0.5108267555,2.2934775744,-0.2286732289,-3.0025128695,2.37$ $30362993,-0.1971573146,-5.079964093,1.0069056107,0.0742933688,-4.28059$ $14602,-1.5959795959,0.076485294,-1.748373781,-2.2128446063,0.190918184$ $7,0.1809974627,-0.3564665855,0.0788169856,0.8024589391,3.6951171777,-0$ $.9239946248,-6.9484627553,1.6009722089,0.6546457231,-5.7345243201,-3.0$ $222947637,0.3266812525,-1.2043473672,-4.1764652618,0.398512427,3.26613$ $92832,-1.3367513429,-0.3435188492,5.1468485042,1.4050334673,0.50262265$ $62,4.577830214,2.1484448212,2.3380980062,7.0859100212,0.7060068036,0.6$ $01922877,5.0484161007,2.8504090736,-0.9697079343 \backslash \mathrm{PG}=\mathrm{C} 01 \quad[\mathrm{X}(\mathrm{C} 7 \mathrm{H} 7 \mathrm{~S} 1)] \backslash N I$ $\operatorname{mag}=0 \backslash \backslash$ 
${ }^{1}$ syn-o-thiomethoxyphenyl cation

$\backslash$

$\backslash 1,1 \backslash \mathrm{C}, 0,-0.5046643696,0.8702498652,-0.1190069907 \backslash \mathrm{C}, 0,-1.6913450434,1$. $4577284423,-0.0203677981 \backslash \mathrm{C}, 0,-2.635205575,0.3928844641,0.0722090241 \backslash \mathrm{C}$, $0,-2.1928697702,-0.932060011,0.0820186809 \backslash \mathrm{C}, 0,-0.8481636323,-1.2503737$ $376,0.0052842893 \backslash \mathrm{C}, 0,0.2059303427,-0.2516850762,-0.0979212354 \backslash \mathrm{H}, 0,-1.9$ $60203219,2.4980556142,-0.1434359115 \backslash \mathrm{H}, 0,-3.6851709998,0.6654186011,0.0$ $461059954 \backslash \mathrm{H}, 0,-2.91521536,-1.7355025068,0.1667183277 \backslash \mathrm{H}, 0,-0.4866197916$ $,-2.270221675,0.1203428656 \backslash \mathrm{S}, 0,1.8936285689,-0.6545618333,-0.063980663$ $1 \backslash \mathrm{C}, 0,2.6657997891,0.9870049051,0.134359282 \backslash \mathrm{H}, 0,2.4304522603,1.4134592$ $967,1.1095708741 \backslash \mathrm{H}, 0,3.7385493712,0.7943510389,0.0735309583 \backslash \mathrm{H}, 0,2.3742$ $004288,1.6496466122,-0.6833936985 \backslash \backslash$ Version=EM64L-G0 9RevB.01\State=1-A MP2 / GTBas $1=-667.2949862 \backslash \mathrm{MP} 4 / \mathrm{GTBas} 1=-667.4019072 \backslash \mathrm{CCSD}(\mathrm{T}) / \mathrm{G} 3 \mathrm{Bas} 1=-667.39$ $93963 \backslash \mathrm{MP} 2 / \mathrm{GTBas} 2=-667.3056605 \backslash \mathrm{MP} 4 / \mathrm{GTBas} 2=-667.4132308 \backslash \mathrm{MP} 2 / \mathrm{GTBas} 3=-667$. $5415125 \backslash \mathrm{MP} 4 / \mathrm{GTBas} 3=-667.6633458 \backslash \mathrm{HF} / \mathrm{GTLargeXP}=-666.4160458 \backslash \mathrm{MP} 2 / \mathrm{GTLargeX}$ $\mathrm{P}=-668.2860699 \backslash \mathrm{HF} / \mathrm{GFHFB} 1=-666.4445465 \backslash \mathrm{HF} / \mathrm{GFHFB} 2=-666.4502545 \backslash \mathrm{G} 4=-668.4$ $688385 \backslash$ FreqCoord $=-0.9536774475,1.6445339124,-0.2248906202,-3.196178928$ $3,2.7547075321,-0.0384895604,-4.9798168406,0.742444039,0.1364552798,-4$ $.1439233107,-1.7613381602,0.1549928446,-1.6027969809,-2.3628639278,0.0$ $099858597,0.3891519501,-0.4756158658,-0.1850443175,-3.7042472488,4.720$ $6409756,-0.2710545903,-6.9639639425,1.2574589198,0.0871277044,-5.50895$ $86488,-3.2796244407,0.3150519807,-0.919578137,-4.2900972266,0.22741505$ $8,3.5784393926,-1.236942602,-0.1209059311,5.0376315265,1.8651689624,0$. $2539022464,4.592889151,2.6710509708,2.096785077,7.0648344459,1.5011059$ $17,0.1389533735,4.4865885949,3.1173803131,-1.2914269312 \backslash \mathrm{PG}=\mathrm{C} 01][\mathrm{X}(\mathrm{C} 7 \mathrm{H} 7$ S1) ] \NImag=0 \

${ }^{1}$ p-thiomethoxyphenyl cation

$\backslash$

$\backslash 1,1 \backslash \mathrm{C}, 0,-0.3711715618,1.1395112024,-0.0410352098 \backslash \mathrm{C}, 0,-1.7405088924,1$. $4890885348,0.0339874933 \backslash \mathrm{C}, 0,-2.4346532005,0.3539088207,-0.0999249607 \backslash \mathrm{C}$ $, 0,-2.3040246981,-0.963949966,0.1064065468 \backslash \mathrm{C}, 0,-0.9225901585,-1.241894$ $2369,0.0500203902 \backslash \mathrm{C}, 0,0.0428504933,-0.2083965404,-0.0194615179 \backslash \mathrm{H}, 0,0.3$ $324586054,1.9552904337,-0.1587485046 \backslash \mathrm{H}, 0,-2.1026898432,2.5081078771,0$. $0713923168 \backslash \mathrm{H}, 0,-3.0684213897,-1.7276954563,0.1612441145 \backslash \mathrm{H}, 0,-0.6287288$ $73,-2.2875336534,0.0111784656 \backslash S, 0,1.7012499227,-0.7373802348,-0.080261$ $2369 \backslash \mathrm{C}, 0,2.6722514067,0.7849323116,0.1070845428 \backslash \mathrm{H}, 0,2.4677621316,1.267$ $1408078,1.0637661267 \backslash \mathrm{H}, 0,3.7121362376,0.4541424852,0.0845674335 \backslash \mathrm{H}, 0,2$.

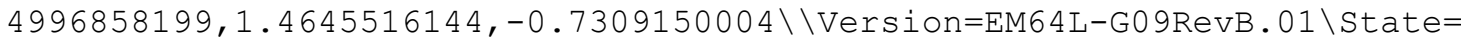
$1-\mathrm{A} \backslash \mathrm{MP} 2 / \mathrm{GTBas} 1=-667.2894464 \backslash \mathrm{MP} 4 / \mathrm{GTBas} 1=-667.3973245 \backslash \mathrm{CCSD}(\mathrm{T}) / \mathrm{G} 3 \mathrm{Bas} 1=-66$ $7.396535 \backslash \mathrm{MP} 2 / \mathrm{GTBas} 2=-667.3003124 \backslash \mathrm{MP} 4 / \mathrm{GTB} a \mathrm{~s} 2=-667.4088182 \backslash \mathrm{MP} 2 / \mathrm{GTBas} 3=-6$ $67.5353596 \backslash \mathrm{MP} 4 / \mathrm{GTBas} 3=-667.6580711 \backslash \mathrm{HF} / \mathrm{GTLargeXP}=-666.4092114 \backslash \mathrm{MP} 2 / \mathrm{GTLar}$ geXP $=-668.2802956 \backslash \mathrm{HF} / \mathrm{GFHFB} 1=-666.4376902 \backslash \mathrm{HF} / \mathrm{GFHFB} 2=-666.4433646 \backslash \mathrm{G} 4=-66$ $8.4653374 \backslash$ FreqCoord=-0.7014126002,2.153364098, $-0.0775453084,-3.2890851$ $386,2.8139695183,0.0642270544,-4.6008277775,0.6687907472,-0.1888308096$ $,-4.3539756828,-1.8216014416,0.2010792322,-1.7434427325,-2.3468399938$, $0.0945248386,0.0809756971,-0.3938123884,-0.036776939,0.6282557148,3.69$ $496343,-0.2999911977,-3.9735079461,4.7396369995,0.1349119268,-5.798476$ $0869,-3.2648712535,0.304707217,-1.1881253818,-4.3228121246,0.021124238$ $5,3.2148964375,-1.3934466996,-0.1516717569,5.0498233169,1.4833071018,0$ $.202360459,4.6633945898,2.3945490986,2.0102266489,7.0149208571,0.85820$ $49224,0.159809289,4.7237216179,2.7676014587,-1.3812291772 \backslash P G=C 01 \quad[X(C 7$ H7S1 ) ] \NImag=0 \\

\footnotetext{
${ }^{1}$ syn-m-methoxyphenyl cation $\backslash$

$\backslash 1,1 \backslash \mathrm{C}, 0,1.2388739653,-1.2117427224,-0.0186943332 \backslash \mathrm{C}, 0,-0.0802180973,-1$
} 
$.1532263875,-0.0491054784 \backslash C, 0,-0.4409603319,0.2550351087,0.0141059292 \backslash$ $\mathrm{C}, 0,0.595700528,1.2227442485,-0.0078035276 \backslash \mathrm{C}, 0,1.9197408959,0.85218221$ $26,-0.0238216376 \backslash \mathrm{C}, 0,2.3628214662,-0.5317958561,0.0405718955 \backslash \mathrm{H}, 0,-0.77$ $3610901,-1.9032537786,-0.4076041595 \backslash \mathrm{H}, 0,0.3153639999,2.2684292668,0.01$ $02839579 \backslash \mathrm{H}, 0,2.706069896,1.592573585,0.0977744835 \backslash \mathrm{H}, 0,3.356947686,-0.8$ $184835805,0.3514997502 \backslash 0,0,-1.6794094484,0.6568228058,-0.1123334112 \backslash \mathrm{C}$, $0,-2.7811768272,-0.2445333921,0.1218362959 \backslash \mathrm{H}, 0,-2.624652815,-0.8029074$ $681,1.0481653976 \backslash \mathrm{H}, 0,-3.6607699515,0.3916819795,0.2015736355 \backslash \mathrm{H}, 0,-2.89$ $34090649,-0.9191500217,-0.7328717977 \backslash \backslash$ Version=EM64L-G0 9RevB.01 \State=1 $-\mathrm{A} \backslash \mathrm{MP} 2 / \mathrm{GTBas} 1=-344.6906472 \backslash \mathrm{MP} 4 / \mathrm{GTBas} 1=-344.7899244 \backslash \mathrm{CCSD}(\mathrm{T}) / \mathrm{G} 3 \mathrm{Bas} 1=-344$ $.7856435 \backslash \mathrm{MP} 2 / \mathrm{GTBas} 2=-344.7038646 \backslash \mathrm{MP} 4 / \mathrm{GTBas} 2=-344.8041173 \backslash \mathrm{MP} 2 / \mathrm{GTBas} 3=-3$ $44.9384061 \backslash \mathrm{MP} 4 / \mathrm{GTBas} 3=-345.0502447 \backslash \mathrm{HF} / \mathrm{GTLargeXP}=-343.7522691 \backslash \mathrm{MP} 2 / \mathrm{GTLar}$ geXP $=-345.4237368 \backslash \mathrm{HF} / \mathrm{GFHFB} 1=-343.7798429 \backslash \mathrm{HF} / \mathrm{GFHFB} 2=-343.7850883 \backslash \mathrm{G} 4=-34$ $5.5889939 \backslash$ FreqCoord $=2.3411325075,-2.2898618888,-0.03532717,-0.15159023$ $49,-2.1792820416,-0.0927959058,-0.8332942627,0.4819465097,0.026656343$, $1.1257108551,2.3106517603,-0.01474653,3.6277845394,1.6103909971,-0.045$ $016371,4.465085472,-1.0049485267,0.0766697711,-1.4619127364,-3.5966284$ $029,-0.770260232,0.595951592,4.286710066,0.0194338639,5.1137309998,3.0$ $095279222,0.1847669966,6.3437117689,-1.5467098114,0.6642382636,-3.1736$ $239225,1.2412152208,-0.2122793827,-5.2556625306,-0.4621011414,0.230237$ $2323,-4.9598750143,-1.5172752247,1.9807455434,-6.9178526438,0.74017167$ $24,0.3809189666,-5.467750723,-1.7369418161,-1.3849269882 \backslash P G=C 01 \quad[X(C 7 H$ 701) $] \backslash N I m a g=0 \backslash \backslash$

${ }^{1}$ syn-o-methoxyphenyl cation

$\backslash$

$\backslash 1,1 \backslash \mathrm{C}, 0,-1.3496507949,1.4112771453,0.0158660867 \backslash \mathrm{C}, 0,-0.1333641303,0.8$ $842959891,-0.0588592095 \backslash \mathrm{C}, 0,0.5700651976,-0.261467636,-0.1445408891 \backslash \mathrm{C}$, $0,-0.4845836408,-1.2462334032,-0.0368105578 \backslash \mathrm{C}, 0,-1.8382120363,-0.95902$ $01907,0.0576979656 \backslash \mathrm{C}, 0,-2.3029498504,0.3527041968,0.1062281906 \backslash \mathrm{H}, 0,-1$. $6303088888,2.4380330795,-0.1897675663 \backslash \mathrm{H}, 0,-0.0847238165,-2.2407260603$, $0.1628763873 \backslash \mathrm{H}, 0,-2.5397257154,-1.7828125018,0.1247611747 \backslash \mathrm{H}, 0,-3.35415$ $98742,0.6153535667,0.0640920245 \backslash 0,0,1.829624787,-0.5904390136,-0.13653$ $22415 \backslash \mathrm{C}, 0,2.7808143738,0.4641899067,0.1642861894 \backslash \mathrm{H}, 0,2.7162811628,0.72$ $66444326,1.2225469017 \backslash \mathrm{H}, 0,3.7596273275,0.051896703,-0.0698169636 \backslash \mathrm{H}, 0,2$ $.5695178991,1.332750786,-0.4711854926 \backslash \backslash$ Version=EM64L-G0 9RevB.01 \State= $1-\mathrm{A} \backslash \mathrm{MP} 2 / \mathrm{GTBas} 1=-344.6747495 \backslash \mathrm{MP} 4 / \mathrm{GTBas} 1=-344.773841 \backslash \mathrm{CCSD}(\mathrm{T}) / \mathrm{G} 3 \mathrm{Bas} 1=-344$ $.7707148 \backslash \mathrm{MP} 2 / \mathrm{GTBas} 2=-344.687961 \backslash \mathrm{MP} 4 / \mathrm{GTBas} 2=-344.788031 \backslash \mathrm{MP} 2 / \mathrm{GTBas} 3=-344$ $.9235212 \backslash \mathrm{MP} 4 / \mathrm{GTBas} 3=-345.0352156 \backslash \mathrm{HF} / \mathrm{GTLargeXP}=-343.7407901 \backslash \mathrm{MP} 2 / \mathrm{GTLarge}$ $\mathrm{XP}=-345.4083784 \backslash \mathrm{HF} / \mathrm{GFHFB} 1=-343.7684919 \backslash \mathrm{HF} / \mathrm{GFHFB} 2=-343.7737507 \backslash \mathrm{G} 4=-345$. $5756294 \backslash$ FreqCoord $=-2.5504703774,2.6669273022,0.0299825587,-0.252021682$ $2,1.6710772397,-0.1112277864,1.0772671014,-0.4941022246,-0.2731426953$, $-0.9157303696,-2.3550398296,-0.0695618731,-3.4737173229,-1.8122855164$, $0.1090333533,-4.351944515,0.6665143378,0.2007421878,-3.0808373119,4.60$ $72148232,-0.3586087292,-0.1601048101,-4.2343585929,0.3077917655,-4.799$ $3860548,-3.3690273746,0.2357644522,-6.3384435681,1.1628497159,0.121116$ $3735,3.4574897733,-1.1157680339,-0.2580085447,5.2549775929,0.877191797$ $2,0.3104559054,5.1330274975,1.3731589737,2.3102788287,7.1046660106,0.0$ $980705559,-0.1319349407,4.8556851229,2.5185339889,-0.8904115387 \backslash \mathrm{PG}=\mathrm{C} 01$

$[\mathrm{X}(\mathrm{C} 7 \mathrm{H} 7 \mathrm{O} 1)] \backslash \mathrm{NImag}=0 \backslash \backslash$

${ }^{1} \mathrm{p}$-methoxyphenyl cation 1

$\backslash 1,1 \backslash C, 0,-1.3650727508,1.477017742,0.0474462369 \backslash C, 0,0.0023365523,1.096$ $7953804,-0.0029210499 \backslash \mathrm{C}, 0,0.3725947355,-0.2646687954,-0.0026882284 \backslash \mathrm{C}, 0$ $,-0.6186082131,-1.276741968,0.0098509026 \backslash C, 0,-1.9980153754,-0.97693464$ $16,0.047964982 \backslash \mathrm{C}, 0,-2.0577886789,0.3494978304,-0.0752335321 \backslash \mathrm{H}, 0,-1.711$ 
$5183802,2.4995404082,0.1118198587 \backslash \mathrm{H}, 0,0.7284947457,1.8987062566,-0.057$ $4542391 \backslash \mathrm{H}, 0,-0.327083474,-2.3196649528,-0.0693582536 \backslash \mathrm{H}, 0,-2.7832589169$ $,-1.7201409309,0.0428914938 \backslash 0,0,1.6214371442,-0.7042262328,-0.02718663$ $8 \backslash \mathrm{C}, 0,2.7114635165,0.2319479282,0.0143098105 \backslash \mathrm{H}, 0,2.6813158901,0.816462$ $8074,0.9385922333 \backslash \mathrm{H}, 0,3.6145058766,-0.3749125245,-0.010929024 \backslash \mathrm{H}, 0,2.68$ 36413283,0.8891296927,-0.8612685528\\Version=EM64L-G09RevB.01 \State=1$\mathrm{A} \backslash \mathrm{MP} 2 / \mathrm{GTBas} 1=-344.6804882 \backslash \mathrm{MP} 4 / \mathrm{GTBas} 1=-344.7789462 \backslash \mathrm{CCSD}(\mathrm{T}) / \mathrm{G} 3 \mathrm{Bas} 1=-344$. $7755012 \backslash \mathrm{MP} 2 / \mathrm{GTBas} 2=-344.6938642 \backslash \mathrm{MP} 4 / \mathrm{GTBas} 2=-344.793301 \backslash \mathrm{MP} 2 / \mathrm{GTB} a \mathrm{~s} 3=-344$ $.9283419 \backslash \mathrm{MP} 4 / \mathrm{GTBas} 3=-345.0394713 \backslash \mathrm{HF} / \mathrm{GTLargeXP}=-343.7494541 \backslash \mathrm{MP} 2 / \mathrm{GTLarge}$ $\mathrm{XP}=-345.4140528 \backslash \mathrm{HF} / \mathrm{GFHFB} 1=-343.7770858 \backslash \mathrm{HF} / \mathrm{GFHFB} 2=-343.7823433 \backslash \mathrm{G} 4=-345$. $5803921 \backslash$ FreqCoord=-2.5796136504,2.7911590259,0.0896603938,0.0044154439 , $2.0726428927,-0.0055199844,0.7041020086,-0.5001515392,-0.0050800155,-$ $1.1690001063,-2.4126926618,0.0186155081,-3.7757018688,-1.8461389224,0$. $09064068,-3.8886570424,0.6604551834,-0.1421707718,-3.2343010099,4.7234$ $468296,0.2113089092,1.3766555586,3.5880348318,-0.108572777,-0.61809818$ $84,-4.3835314808,-0.1310681043,-5.2595971098,-3.2505952694,0.081053176$ $7,3.0640721443,-1.3307947156,-0.0513753004,5.1239234654,0.4383180615,0$ $.027041623,5.0669527081,1.5428911037,1.7736822714,6.8304262125,-0.7084$ $81995,-0.0206528622,5.0713471494,1.6802116158,-1.6275616917 \backslash \mathrm{PG}=\mathrm{C} 01 \quad[\mathrm{X}($ $\mathrm{C} 7 \mathrm{H} 7 \mathrm{O} 1)] \backslash \mathrm{NImag}=0 \backslash \backslash$

${ }^{1}$ anti-o-thiomethoxyphenyl cation

$\backslash$

$\backslash 1,1 \backslash \mathrm{C}, 0,-3.4992199216,3.6064392283,-0.0008174707 \backslash \mathrm{C}, 0,-4.707624665,2.9$ $242740382,-0.0004417656 \backslash \mathrm{C}, 0,-2.1961459093,2.9596243628,-0.0019297461 \backslash \mathrm{C}$ $, 0,-4.7631747504,1.5316445136,-0.0010966216 \backslash \mathrm{C}, 0,-3.5483733264,0.784696$ $0274,-0.0021315737 \backslash \mathrm{C}, 0,-2.5788397035,1.6882666641,-0.0015031015 \backslash \mathrm{H}, 0,-5$ $.6908457268,0.9698934717,0.000660632 \backslash S, 0,-0.6088696546,3.6799300313,-0$ $.0057279607 \backslash \mathrm{H}, 0,-3.4591316596,4.6917418727,-0.002202989 \backslash \mathrm{H}, 0,-5.6268254$ $876,3.498071948,0.0000013614 \backslash \mathrm{C}, 0,-0.9850207681,5.4586448015,-0.0031566$ $873 \backslash \mathrm{H}, 0,0.0019160501,5.9275887237,-0.0043373618 \backslash \mathrm{H}, 0,-1.5152268666,5.75$ $13035735,0.9040770115 \backslash \mathrm{H}, 0,-1.5182784137,5.7532155829,-0.9079929082 \backslash \mathrm{H}, 0$ $,-3.486059197,-0.2949748398,-0.0009408187 \backslash \backslash$ Version=EM64L-G09RevB.01\St ate $=1-\mathrm{A} \backslash \mathrm{MP} 2 / \mathrm{GTBas} 1=-667.2951367 \backslash \mathrm{MP} 4 / \mathrm{GTBas} 1=-667.4019091 \backslash \mathrm{CCSD}(\mathrm{T}) / \mathrm{G} 3 \mathrm{Bas} 1$ $=-667.3992662 \backslash \mathrm{MP} 2 / \mathrm{GTBas} 2=-667.3055411 \backslash \mathrm{MP} 4 / \mathrm{GTBas} 2=-667.4129671 \backslash \mathrm{MP} 2 / \mathrm{GTBa}$ $\mathrm{s} 3=-667.5421081 \backslash \mathrm{MP} 4 / \mathrm{GTBas} 3=-667.6637977 \backslash \mathrm{HF} / \mathrm{GTLargeXP}=-666.4166724 \backslash \mathrm{MP} 2 /$ GTLargeXP $=-668.2866861 \backslash \mathrm{HF} / \mathrm{GFHFB} 1=-666.4452291 \backslash \mathrm{HF} / \mathrm{GFHFB} 2=-666.4509298 \backslash \mathrm{G}$ $4=-668.4691106 \backslash$ FreqCoord $=-6.6125673306,6.8151824564,-0.0015447957,-8.8$ $961213533,5.5260770697,-0.000834816,-4.1501143164,5.5928795019,-0.0036$ $466916,-9.0010958013,2.8943886636,-0.0020723145,-6.7054538041,1.482860$ $5893,-0.0040280905,-4.8733007802,3.1903616344,-0.0028404502,-10.754139$ $8882,1.8328330396,0.0012484136,-1.1505968978,6.9540599473,-0.010824277$ $,-6.5368114942,8.8661072256,-0.0041630459,-10.6331591691,6.6103979748$, $0.0000025727,-1.8614194869,10.3153437315,-0.0059652745,0.0036208099,11$ $.2015193162,-0.0081964259,-2.8633638071,10.868388661,1.7084579548,-2.8$ $691303954,10.8720018351,-1.7158579271,-6.5876971654,-0.5574216633,-0.0$ $017778897 \backslash \mathrm{PG}=\mathrm{C} 01 \quad[\mathrm{X}(\mathrm{C} 7 \mathrm{H} 7 \mathrm{~S} 1)] \backslash \mathrm{NImag}=0 \backslash \backslash$

${ }^{1}$ anti-o-methoxyphenyl cation

$\backslash$

$\backslash 1,1 \backslash \mathrm{C}, 0,-1.3301721333,1.4526716129,-0.0716182421 \backslash \mathrm{C}, 0,-0.1744739872,0$. $8084934329,-0.0269319118 \backslash \mathrm{C}, 0,0.4633363969,-0.3756641691,0.0575972144 \backslash \mathrm{C}$ $, 0,-0.6642826828,-1.2730152708,0.1239356493 \backslash \mathrm{C}, 0,-1.994891872,-0.875179$ $7593,0.0974850261 \backslash \mathrm{C}, 0,-2.3563027158,0.4647835568,0.0013894302 \backslash \mathrm{H}, 0,-1.5$ $09720158,2.5178097842,-0.145883729 \backslash \mathrm{H}, 0,-0.3845826355,-2.3218055738,0.1$ $966341949 \backslash \mathrm{H}, 0,-2.7645455331,-1.6358809663,0.153431871 \backslash \mathrm{H}, 0,-3.382754839$ $4,0.8131495236,-0.020543219 \backslash 0,0,1.7438278607,-0.6410817483,0.073013499$ 
$5 \backslash \mathrm{C}, 0,2.183547193,-2.0175480432,0.1721677194 \backslash \mathrm{H}, 0,1.83669469,-2.4581069$ $879,1.1102850305 \backslash \mathrm{H}, 0,3.2699337666,-1.9565034815,0.1657493035 \backslash \mathrm{H}, 0,1.833$ $49665,-2.5888919102,-0.6912618369 \backslash \backslash$ Version=EM64L-G0 9RevB. $01 \backslash$ State=1-A MP2 / GTBas $1=-344.6721076 \backslash \mathrm{MP} 4 / \mathrm{GTBas} 1=-344.7709249 \backslash \mathrm{CCSD}(\mathrm{T}) / \mathrm{G} 3 \mathrm{Bas} 1=-344.76$ $76937 \backslash \mathrm{MP} 2 / \mathrm{GTBas} 2=-344.6851475 \backslash \mathrm{MP} 4 / \mathrm{GTBas} 2=-344.7849207 \backslash \mathrm{MP} 2 / \mathrm{GTBas} 3=-344$. $921696 \backslash \mathrm{MP} 4 / \mathrm{GTBas} 3=-345.0331923 \backslash \mathrm{HF} / \mathrm{GTLargeXP}=-343.7439568 \backslash \mathrm{MP} 2 / \mathrm{GTLargeXP}$ $=-345.406663 \backslash \mathrm{HF} / \mathrm{GFHFB} 1=-343.7717546 \backslash \mathrm{HF} / \mathrm{GFHFB} 2=-343.7770204 \backslash \mathrm{G} 4=-345.574$ $2316 \backslash$ FreqCoord=-2 .5136610415,2.7451515094, $-0.1353388636,-0.3297080531$, $1.5278311684,-0.0508939375,0.8755788975,-0.7099023976,0.1088429613,-1$. $2553123453,-2.4056502248,0.2342044353,-3.7697993028,-1.6538500622,0.18$ $42200013,-4.4527668191,0.8783136333,0.0026256426,-2.8529576359,4.75797$ $09469,-0.2756802951,-0.7267558566,-4.3875766682,0.3715847767,-5.224233$ $9394,-3.0913670123,0.2899442162,-6.3924802212,1.5366299047,-0.03882105$ $78,3.2953570796,-1.2114689331,0.1379755181,4.126306193,-3.8126132617,0$ $.3253498386,3.4708499538,-4.6451490124,2.098134637,6.1792792915,-3.697$ $255758,0.3132207902,3.4648065341,-4.8922966979,-1.3062955579 \backslash P G=C 01] \mathrm{X}$ (C7H7O1) ] \NImag=1 \\

${ }^{1} \mathrm{p}$-hydroxyphenyl cation

$\backslash \backslash 1,1 \backslash \mathrm{C}, 0,-2.6811752068,2.4002677697,0 . \backslash \mathrm{C}, 0,-3.942399206,1.9997214288$, $0 . \backslash C, 0,-1.4010138634,2.0545011052,0 . \backslash C, 0,-1.424832751,0.6349649388,0 . \backslash$ $\mathrm{C}, 0,-3.8668838879,0.5763811331,0 . \backslash \mathrm{C}, 0,-2.6310107057,-0.0932483077,0 . \backslash \mathrm{H}$ $, 0,-4.8656920729,2.5621246569,0 . \backslash \mathrm{H}, 0,-0.4993999382,2.6508199059,0 . \backslash \mathrm{H}, 0$ $,-0.468384535,0.1210320324,0 . \backslash \mathrm{H}, 0,-4.8151916248,0.046708576,0 . \backslash 0,0,-2$. $5113094911,-1.4226968476,0 . \backslash \mathrm{H}, 0,-3.3652267172,-1.8731163915,0 . \backslash \backslash$ Versio n=EM64L-G09RevB. $01 \backslash$ State=1-A' \MP2/GTBas $1=-305.5205722 \backslash \mathrm{MP} 4 / \mathrm{GTBas} 1=-305$. $6012047 \backslash \mathrm{CCSD}(\mathrm{T}) / \mathrm{G} 3 \mathrm{Bas} 1=-305.5979012 \backslash \mathrm{MP} 2 / \mathrm{GTBas} 2=-305.5328747 \backslash \mathrm{MP} 4 / \mathrm{GTBas} 2$ $=-305.6142322 \backslash \mathrm{MP} 2 / \mathrm{GTB} a \mathrm{~s} 3=-305.7362627 \backslash \mathrm{MP} 4 / \mathrm{GTB} a \mathrm{~s} 3=-305.8269237 \backslash \mathrm{HF} / \mathrm{GTLar}$ geXP $=-304.7124769 \backslash \mathrm{MP} 2 / \mathrm{GTLargeXP}=-306.1630927 \backslash \mathrm{HF} / \mathrm{GFHFB} 1=-304.7376542 \backslash \mathrm{HF}$ $/ \mathrm{GFHFB} 2=-304.7423736 \backslash \mathrm{G} 4=-306.313366 \backslash$ FreqCoord $=-5.0666868552,4.53584873$ $02,0 .,-7.4500548058,3.7789258424,0 .,-2.6475325102,3.8824444286,0 .,-2.6$ $925436846,1.1999098383,0 .,-7.3073515357,1.0892024897,0 .,-4.9718896864$, $-0.1762137639,0 .,-9.1948254646,4.8417139199,0 .,-0.943729114,5.00932364$ $98,0 .,-0.885118496,0.2287173945,0 .,-9.0993934483,0.0882664167,0 .,-4.74$ $56871731,-2.688507412,0 .,-6.3593568706,-3.539676995,0 . \backslash P G=C S \quad[S G(C 6 H 50$ 1) ] \NImag=1 \\}

${ }^{1}$ p-thiolphenyl cation

$\backslash \backslash 1,1 \backslash \mathrm{C}, 0,-2.6838883582,2.4606269558,0 . \backslash \mathrm{C}, 0,-3.9407277426,2.0510547321$ $, 0 . \backslash \mathrm{C}, 0,-1.404990165,2.1186880834,0 . \backslash \mathrm{C}, 0,-1.4239211416,0.6932976895,0$. $\backslash \mathrm{C}, 0,-3.8510057523,0.6246110789,0 . \backslash \mathrm{C}, 0,-2.6192955458,-0.0481203797,0 . \backslash$ $\mathrm{H}, \mathrm{O},-4.8728773476,2.5982454514, \mathrm{O} . \backslash \mathrm{H}, \mathrm{O},-0.5027868431,2.7138253121,0 . \backslash \mathrm{H}$, $0,-0.4481494577,0.2153105773,0 . \backslash \mathrm{H}, 0,-4.8037491541,0.1044232537,0 . \backslash \mathrm{S}, 0$, $-2.4772330926,-1.8041702168,0 . \backslash \mathrm{H}, 0,-3.7957253995,-2.0714125376,0 . \backslash \backslash \mathrm{Ver}$ sion=EM64L-G09RevB.01 \State=1-A ' \MP2/GTBas $1=-628.1163657 \backslash \mathrm{MP} 4 / \mathrm{GTB}$ s $1=-6$ $28.2078129 \backslash \mathrm{CCSD}(\mathrm{T}) / \mathrm{G} 3 \mathrm{Bas} 1=-628.2053818 \backslash \mathrm{MP} 2 / \mathrm{GTB} a \mathrm{~s} 2=-628.1252095 \backslash \mathrm{MP} 4 / \mathrm{GTB}$ as $2=-628.2170677 \backslash \mathrm{MP} 2 / \mathrm{GTBas} 3=-628.3277777 \backslash \mathrm{MP} 4 / \mathrm{GTBas} 3=-628.4316546 \backslash \mathrm{HF} / \mathrm{GT}$ LargeXP $=-627.3621182 \backslash \mathrm{MP} 2 / \mathrm{GTLargeXP}=-629.0130092 \backslash \mathrm{HF} / \mathrm{GFHFB} 1=-627.3876688$ $\backslash \mathrm{HF} / \mathrm{GFHFB} 2=-627.3926277 \backslash \mathrm{G} 4=-629.1831724 \backslash$ FreqCoord $=-5.0718139682,4.6499$ $110616,0 .,-7.4468961979,3.8759317272,0 .,-2.6550466313,4.0037402385,0 .$, $-2.6908209925,1.3101427617,0 .,-7.2773462079,1.1803438787,0 .,-4.9497512$ $426,-0.0909343391,0 .,-9.208403666,4.9099723291,0 .,-0.9501294366,5.1283$ $866123,0 .,-0.8468797416,0.4068780246,0 .,-9.0777703123,0.1973313513,0$. , $-4.6812921124,-3.4093876069,0 .,-7.1728814807,-3.9144024042,0 . \backslash P G=C S \quad[S$ $\mathrm{G}(\mathrm{C} 6 \mathrm{H} 5 \mathrm{~S} 1)] \backslash \mathrm{NImag}=1 \backslash \backslash$ 
${ }^{1}$ m-nitrophenyl cation

$\backslash \backslash 1,1 \backslash \mathrm{C}, 0,-2.7610183094,2.8721114291,0 . \backslash \mathrm{C}, 0,-3.9537348772,2.16402502$

$76,0 . \backslash C, 0,-1.4726979751,2.2665465906,0 . \backslash C, 0,-1.7589377474,0.979971661$, $0 . \backslash C, 0,-3.9293398255,0.7688373368,0 . \backslash C, 0,-2.7000500088,0.042610576,0 . \backslash$ $\mathrm{N}, 0,-2.7413169376,4.3902235742,0 . \backslash \mathrm{H}, 0,-4.8907770687,2.7057033928,0 . \backslash \mathrm{H}$, $0,-4.8408873514,0.1772551258,0 . \backslash \mathrm{H}, 0,-2.6214398229,-1.0365747911,0 . \backslash \mathrm{H}, \mathrm{O}$ $,-0.5611400738,2.8550350268,0 . \backslash 0,0,-1.6529685137,4.9196598233,0 . \backslash 0,0,-$ $3.8382314886,4.9029152272,0 . \backslash \backslash$ Version=EM64L-G0 9RevB.01 \State=1-A '\MP2/ GTBas $1=-434.473369 \backslash \mathrm{MP} 4 / \mathrm{GTBas} 1=-434.5701017 \backslash \mathrm{CCSD}(\mathrm{T}) / \mathrm{G} 3 \mathrm{Bas} 1=-434.5565294$ $\backslash \mathrm{MP} 2 / \mathrm{GTBas} 2=-434.4930856 \backslash \mathrm{MP} 4 / \mathrm{GTBas} 2=-434.5912709 \backslash \mathrm{MP} 2 / \mathrm{GTB} a \mathrm{~s} 3=-434.75607$ $32 \backslash \mathrm{MP} 4 / \mathrm{GTBas} 3=-434.8676999 \backslash \mathrm{HF} / \mathrm{GTLargeXP}=-433.3370079 \backslash \mathrm{MP} 2 / \mathrm{GTLargeXP}=-43$ $5.3321634 \backslash \mathrm{HF} / \mathrm{GFHFB} 1=-433.3725417 \backslash \mathrm{HF} / \mathrm{GFHFB} 2=-433.379274 \backslash \mathrm{G} 4=-435.5423377$ $\backslash$ FreqCoord $=-5.2175684527,5.4275040242,0 .,-7.4714761199,4.0894146469,0$. $,-2.7829958494,4.2831523236,0 .,-3.3239106274,1.8518780573,0 .,-7.425376$ $1532,1.4528920073,0 .,-5.1023550617,0.0805223189,0 .,-5.1803382554,8.296$ $3202173,0 .,-9.2422292369,5.1130384091,0 .,-9.1479513344,0.3349636434,0$. $,-4.9538033392,-1.9588424715,0 .,-1.0604010616,5.3952343004,0 .,-3.12365$ $77971,9.2968097329,0 .,-7.253206348,9.2651670323,0 . \backslash P G=C S \quad[S G(C 6 H 4 N 102)$ ] \NImag=0 \\

${ }^{1}$ o-nitrophenyl cation

$\backslash \backslash 1,1 \backslash C, 0,-3.1648799594,3.2243110685,0 . \backslash C, 0,-4.0703364861,2.22641418$ $97,0 . \backslash C, 0,-1.7721747212,2.8850893482,0 . \backslash C, 0,-1.2424862074,1.2178612568$ $, 0 . \backslash C, 0,-3.6831299781,0.8311304955,0 . \backslash C, 0,-2.4096701786,0.3729143525,0$ $. \backslash \mathrm{H}, 0,-3.4325242091,4.2742500068,0 . \backslash \mathrm{H}, 0,-5.1274449832,2.463681471,0 . \backslash \mathrm{N}$ $, 0,-0.7089257566,3.5097114719,0 . \backslash \mathrm{H}, 0,-4.4808941823,0.0948175065,0 . \backslash \mathrm{H}, \mathrm{O}$ $,-2.1715362512,-0.6864029241,0 . \backslash 0,0,-0.0809217074,1.0269407465,0 . \backslash 0,0$,

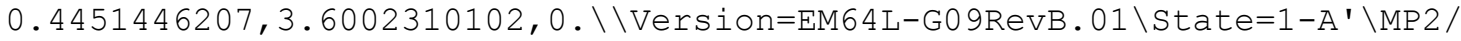
GTBas $1=-434.5528987 \backslash \mathrm{MP} 4 / \mathrm{GTBas} 1=-434.6561643 \backslash \mathrm{CCSD}(\mathrm{T}) / \mathrm{G} 3 \mathrm{Bas} 1=-434.640625$ $4 \backslash \mathrm{MP} 2 / \mathrm{GTBas} 2=-434.5700765 \backslash \mathrm{MP} 4 / \mathrm{GTBas} 2=-434.674822 \backslash \mathrm{MP} 2 / \mathrm{GTB} a \mathrm{~s} 3=-434.83525$ $95 \backslash \mathrm{MP} 4 / \mathrm{GTBas} 3=-434.9533967 \backslash \mathrm{HF} / \mathrm{GTLargeXP}=-433.4056884 \backslash \mathrm{MP} 2 / \mathrm{GTLargeXP}=-43$ $5.4112168 \backslash \mathrm{HF} / \mathrm{GFHFB} 1=-433.4404991 \backslash \mathrm{HF} / \mathrm{GFHFB} 2=-433.4470053 \backslash \mathrm{G} 4=-435.623629$ $\backslash$ FreqCoord $=-5.9807563667,6.0930648866,0 .,-7.6918212274,4.2073130769,0$. $,-3.3489248826,5.452028737,0 .,-2.3479586559,2.3014242432,0 .,-6.9601069$ $704,1.5706090171,0 .,-4.553616708,0.7047059972,0 .,-6.4865306997,8.07716$ $19363,0 .,-9.6894667798,4.6556832589,0 .,-1.3396755286,6.6323934873,0 .,-$ $8.4676628351,0.17917912,0 .,-4.1036088025,-1.2971135434,0 .,-0.152919865$ $1,1.9406367657,0 ., 0.8412014226,6.8034506243,0 . \backslash P G=C S \quad[S G(C 6 H 4 N 1 O 2)] \backslash N I$ $\operatorname{mag}=0 \backslash \backslash$

${ }^{1}$ p-nitrophenyl cation

$\backslash \backslash 1,1 \backslash \mathrm{C}, 0,-2.7003694602,2.8889393311,0 . \backslash \mathrm{C}, 0,-3.8638823653,2.12544737$

$93,0 . \backslash \mathrm{C}, 0,-1.4231787822,2.254165628,0 . \backslash \mathrm{C}, 0,-1.7256186434,0.9643184346$, $0 . \backslash C, 0,-3.8706514235,0.7338169447,0 . \backslash C, 0,-2.6427540922,0.0082658428,0$. $\backslash \mathrm{N}, 0,-5.1712177749,2.8353611484,0 . \backslash 0,0,-5.1198357809,4.0505525674,0 . \backslash 0$ $, 0,-6.1624528359,2.1305322168,0 . \backslash \mathrm{H}, 0,-2.7408791092,3.974894145,0 . \backslash \mathrm{H}, 0$, $-0.4711566335,2.7685743844,0 . \backslash \mathrm{H}, 0,-4.8035313994,0.1764354654,0 . \backslash \mathrm{H}, 0,-2$ $.5557816993,-1.0703434878,0 . \backslash \backslash$ Version=EM64L-G09RevB.01 \State=1-A $\backslash$ MP2/ GTBas $1=-434.4731159 \backslash \mathrm{MP} 4 / \mathrm{GTBas} 1=-434.5685429 \backslash \mathrm{CCSD}(\mathrm{T}) / \mathrm{G} 3 \mathrm{Bas} 1=-434.555032$ $2 \backslash \mathrm{MP} 2 / \mathrm{GTBas} 2=-434.4931823 \backslash \mathrm{MP} 4 / \mathrm{GTBas} 2=-434.5900671 \backslash \mathrm{MP} 2 / \mathrm{GTB} a \mathrm{~s} 3=-434.7561$ $778 \backslash \mathrm{MP} 4 / \mathrm{GTBas} 3=-434.8666054 \backslash \mathrm{HF} / \mathrm{GTL}$ LargeXP $=-433.3395782 \backslash \mathrm{MP} 2 / \mathrm{GTL}$ LargeXP $=-4$ $35.3325086 \backslash \mathrm{HF} / \mathrm{GFHFB} 1=-433.3751376 \backslash \mathrm{HF} / \mathrm{GFHFB} 2=-433.3818702 \backslash \mathrm{G} 4=-435.54180$ $85 \backslash$ FreqCoord $=-5.1029587375,5.4593041503,0 .,-7.3016794801,4.0165134566$, $0 .,-2.6894181365,4.259755695,0 .,-3.2609466458,1.8222977462,0,-7.31447$ $11463,1.3867130572,0 .,-4.9940814708,0.0156201792,0 .,-9.7721853681,5.35$ $80560582,0 .,-9.6750874713,7.6544350393,0 .,-11.6453481666,4.0261224071$, $0 .,-5.1795108798,7.5114613412,0 .,-0.890357003,5.231847365,0 \ldots,-9.077358$ 
$8157,0.3334147097,0 .,-4.8297274671,-2.02265606,0 . \backslash \mathrm{PG}=\mathrm{CS} \quad[\mathrm{SG}(\mathrm{C} 6 \mathrm{H} 4 \mathrm{~N} 1 \mathrm{O} 2)]$ $\backslash$ NImag $=0 \backslash \backslash$

${ }^{3}$ m-aminophenyl cation

$\backslash \backslash 1,3 \backslash \mathrm{C}, 0,-3.012502461,2.9774953019,-0.4100054141 \backslash \mathrm{C}, 0,-3.9990574206$, $2.0381000612,-0.8766120765 \backslash \mathrm{C}, 0,-1.778051988,2.5006413444,0.1567844014 \backslash$ $\mathrm{C}, 0,-1.5311133054,1.1538209682,0.2563621245 \backslash \mathrm{C}, 0,-3.6731794654,0.735685$ $0652,-0.7400855505 \backslash \mathrm{C}, 0,-2.4953373767,0.2202259151,-0.2004562794 \backslash \mathrm{H}, 0,-1$ $.0486464364,3.2253105612,0.5028522465 \backslash \mathrm{H}, 0,-2.3163766234,-0.8474498195$, $-0.1295748674 \backslash \mathrm{H}, 0,-0.600380599,0.7982162402,0.683755199 \backslash \mathrm{H}, 0,-4.9345415$ $367,2.3830595201,-1.305512685 \backslash \mathrm{N}, 0,-3.2345800205,4.2873621097,-0.499858$ $6823 \backslash \mathrm{H}, 0,-4.0921926654,4.6541321671,-0.8939409571 \backslash \mathrm{H}, 0,-2.5539601015,4$. $9647505652,-0.178417459 \backslash \backslash$ Version=EM64L-G09RevB. $01 \backslash$ state=3-A $\backslash \mathrm{MP} 2 / \mathrm{GTB} a \mathrm{~s} 1$ $=-285.6884128 \backslash \mathrm{MP} 4 / \mathrm{GTBas} 1=-285.7817111 \backslash \mathrm{CCSD}(\mathrm{T}) / \mathrm{G} 3 \mathrm{Bas} 1=-285.7921899 \backslash \mathrm{MP} 2 /$ GTBas $2=-285.6989484 \backslash \mathrm{MP} 4 / \mathrm{GTBas} 2=-285.7926621 \backslash \mathrm{MP} 2 / \mathrm{GTBas} 3=-285.8863124 \backslash \mathrm{MP}$ $4 / \mathrm{GTBas} 3=-285.9902904 \backslash \mathrm{HF} / \mathrm{GTLargeXP}=-284.9383895 \backslash \mathrm{MP} 2 / \mathrm{GTLargeXP}=-286.299$ $0331 \backslash \mathrm{HF} / \mathrm{GFHFB} 1=-284.9612684 \backslash \mathrm{HF} / \mathrm{GFHFB} 2=-284.9654653 \backslash \mathrm{G} 4=-286.4581553 \backslash \mathrm{Fre}$ qCoord $=-5.6928046259,5.6266506825,-0.7747979457,-7.5571233146,3.851450$ $9472,-1.6565567493,-3.3600313073,4.7255272975,0.2962795805,-2.89338482$ $56,2.1804056363,0.4844542062,-6.9413032266,1.3902432932,-1.3985590054$, $-4.7155042511,0.4161666669,-0.3788074697,-1.981654575,6.0949536542,0.9$ $502530311,-4.3773174389,-1.6014480703,-0.244861013,-1.1345549076,1.508$ $4100889,1.292110068,-9.3249320958,4.5033298513,-2.4670614377,-6.112470$ $3937,8.1019402198,-0.9445960147,-7.7331234206,8.795035182,-1.689303587$ $8,-4.8262851461,9.3820188864,-0.3371601349 \backslash \mathrm{PG}=\mathrm{C} 01 \quad[\mathrm{X}(\mathrm{C} 6 \mathrm{H} 6 \mathrm{~N} 1)] \backslash \mathrm{NImag}=0 \backslash$ $\backslash$

${ }^{3}$ o-aminophenyl cation

$\backslash \backslash 1,3 \backslash \mathrm{C}, 0,-2.7297030942,2.6886066899,-0.1823857369 \backslash \mathrm{C}, 0,-3.9459441385$ $, 1.9590023773,-0.1391233144 \backslash \mathrm{C}, 0,-1.5126247413,1.9214679656,-0.19560416$ $26 \backslash \mathrm{C}, 0,-1.5879346467,0.5545075316,-0.1666186993 \backslash \mathrm{C}, 0,-4.0547107831,0.60$ $90871923,-0.1100449167 \backslash \mathrm{C}, 0,-2.8392904334,-0.1155629924,-0.1241181729 \backslash \mathrm{H}$ $, 0,-0.5590700568,2.4390460092,-0.2277133589 \backslash \mathrm{H}, 0,-5.0104834216,0.097849$ $2582,-0.0772779806 \backslash \mathrm{H}, 0,-2.8657850535,-1.2001336173,-0.102254293 \backslash \mathrm{H}, 0,-0$ $.6778227465,-0.033930773,-0.1759598731 \backslash \mathrm{N}, 0,-2.724411636,4.0173282883,-$ $0.2101100112 \backslash \mathrm{H}, 0,-3.591087753,4.5417138694,-0.2008273767 \backslash \mathrm{H}, 0,-1.860101$ $4954,4.5439482008,-0.2418221038 \backslash \backslash$ Version=EM64L-G0 9RevB .01 $\backslash$ State $=3-A \backslash M P$ $2 / \mathrm{GTBas} 1=-285.6827663 \backslash \mathrm{MP} 4 / \mathrm{GTBas} 1=-285.7778071 \backslash \mathrm{CCSD}(\mathrm{T}) / \mathrm{G} 3 \mathrm{Bas} 1=-285.7948$ $685 \backslash \mathrm{MP} 2 / \mathrm{GTBas} 2=-285.6930577 \backslash \mathrm{MP} 4 / \mathrm{GTB} a \mathrm{~s} 2=-285.7886926 \backslash \mathrm{MP} 2 / \mathrm{GTB}$ s $3=-285.87$ $96961 \backslash \mathrm{MP} 4 / \mathrm{GTBas} 3=-285.9857583 \backslash \mathrm{HF} / \mathrm{GTLargeXP}=-284.9525801 \backslash \mathrm{MP} 2 / \mathrm{GTLargeXP}=$ $-286.2917115 \backslash \mathrm{HF} / \mathrm{GFHFB} 1=-284.9755059 \backslash \mathrm{HF} / \mathrm{GFHFB} 2=-284.979702 \backslash \mathrm{G} 4=-286.4594$ $656 \backslash$ FreqCoord=-5.1583912721, 5.080730323, -0.3446590933, -7.4567537574,3. $7019779868,-0.2629049628,-2.8584465028,3.631048228,-0.3696382978,-3.00$ $07615992,1.0478673734,-0.3148637102,-7.6622929282,1.1510079845,-0.2079$ $547549,-5.3654813309,-0.2183824067,-0.234549355,-1.0564892965,4.609128$ $9828,-0.4303158851,-9.4684414602,0.1849083003,-0.1460342194,-5.4155489$ $068,-2.2679238595,-0.1932326096,-1.2808993575,-0.0641198684,-0.3325159$ $705,-5.1483918653,7.5916502508,-0.3970503789,-6.7861723722,8.582595387$ $2,-0.379508742,-3.5150824057,8.5868176615,-0.456977549 \backslash \mathrm{PG}=\mathrm{C} 01 \quad[\mathrm{X}(\mathrm{C} 6 \mathrm{H} 6 \mathrm{~N}$ 1) ] \NImag=0 \\

${ }^{3} \mathrm{p}$-aminophenyl cation

$\backslash \backslash 1,3 \backslash \mathrm{C}, 0,-2.8831529335,3.0594277661,-0.2626257181 \backslash \mathrm{C}, 0,-4.0996385674$ $, 2.3057963082,-0.1280881455 \backslash \mathrm{C}, 0,-1.7174817115,2.4076889786,-0.59166435$ $78 \backslash \mathrm{C}, 0,-1.8046806035,1.0288215671,-0.7747655093 \backslash \mathrm{C}, 0,-4.1138364693,0.88$ $31953767,-0.3327867381 \backslash \mathrm{C}, 0,-2.9454704986,0.2362220572,-0.6616732067 \backslash \mathrm{H}$, $0,-2.9023956185,4.1328713203,-0.1023353161 \backslash \mathrm{H}, 0,-0.7808431022,2.9426088$ 
$196,-0.7014752974 \backslash \mathrm{H}, 0,-5.0473627779,0.3398974535,-0.2246187929 \backslash \mathrm{H}, 0,-2$. $9174049966,-0.8354983973,-0.8232810525 \backslash N, 0,-5.229794993,2.9346138077,0$ $.1913702103 \backslash \mathrm{H}, 0,-5.2557211328,3.9351643481,0.343485494 \backslash \mathrm{H}, 0,-6.10412659$ $52,2.4349205942,0.2951184303 \backslash \backslash$ Version=EM64L-G09RevB.01 State=3-A \MP $2 / \mathrm{G}$ TBas $1=-285.685837 \backslash \mathrm{MP} 4 / \mathrm{GTBas} 1=-285.780324 \backslash \mathrm{CCSD}(\mathrm{T}) / \mathrm{G} 3 \mathrm{Bas} 1=-285.7969015 \backslash \mathrm{M}$ $\mathrm{P} 2 / \mathrm{GTBas} 2=-285.6959956 \backslash \mathrm{MP} 4 / \mathrm{GTBas} 2=-285.7910552 \backslash \mathrm{MP} 2 / \mathrm{GTB}$ s $3=-285.8829698$ $\backslash \mathrm{MP} 4 / \mathrm{GTBas} 3=-285.9884455 \backslash \mathrm{HF} / \mathrm{GTLargeXP}=-284.9544215 \backslash \mathrm{MP} 2 / \mathrm{GTLargeXP}=-286$. $2950469 \backslash \mathrm{HF} / \mathrm{GFHFB} 1=-284.9773335 \backslash \mathrm{HF} / \mathrm{GFHFB} 2=-284.9815357 \backslash \mathrm{G} 4=-286.4615998 \backslash$ FreqCoord $=-5.4483694435,5.7814806012,-0.4962906827,-7.7471941361,4.357$ $3235408,-0.2420515159,-3.2455700729,4.5498727827,-1.1180835989,-3.4103$ $52098,1.9441910014,-1.4640946299,-7.7740242824,1.6689973838,-0.6288757$ $957,-5.5661325749,0.4463949947,-1.2503811501,-5.4847328483,7.809994937$ $8,-0.1933857212,-1.4755796159,5.5607247853,-1.3255962011,-9.5381333436$ $, 0.6423131003,-0.4244680029,-5.5130964622,-1.5788631554,-1.5557757196$, $-9.882880268,5.5456164024,0.3616372875,-9.9318735719,7.4363829058,0.64$ $90935143,-11.5351275453,4.6013330784,0.55769301 \backslash \mathrm{PG}=\mathrm{C} 01 \quad[\mathrm{X}(\mathrm{C} 6 \mathrm{H} 6 \mathrm{~N} 1)] \backslash \mathrm{NIm}$ $\operatorname{ag}=0 \backslash \backslash$

${ }^{3}$ m-cyanophenyl cation

$\backslash \backslash 1,3 \backslash \mathrm{C}, 0,-3.0025665104,3.0909873368,-0.5330527741 \backslash \mathrm{C}, 0,-4.0070352201$

$, 2.1863629301,-0.9764919231 \backslash \mathrm{C}, 0,-1.7734867085,2.6047231777,0.075395707$ $9 \backslash \mathrm{C}, 0,-1.5539647422,1.2552050407,0.2362582688 \backslash \mathrm{C}, 0,-3.7144941412,0.8770$ $191857,-0.7823363401 \backslash \mathrm{C}, 0,-2.5414968014,0.3479116486,-0.1989637387 \backslash \mathrm{H}, 0$, $-1.0391644336,3.3353894691,0.3967088511 \backslash \mathrm{H}, 0,-2.4076189636,-0.724922118$ $2,-0.0905343776 \backslash \mathrm{H}, 0,-0.6390952501,0.8909155009,0.6892553171 \backslash \mathrm{H}, 0,-4.924$ $3426715,2.5443585234,-1.4303958795 \backslash \mathrm{C}, 0,-3.1761407207,4.4764304663,-0.6$ $734063838 \backslash \mathrm{N}, 0,-3.3134938367,5.6309288389,-0.7868767281 \backslash \backslash$ Version=EM64LG09RevB.01 State=3-A \MP2/GTBas $1=-322.4166353 \backslash \mathrm{MP} 4 / \mathrm{GTBas} 1=-322.5210979 \backslash \mathrm{C}$ $\mathrm{CSD}(\mathrm{T}) / \mathrm{G} 3 \mathrm{Bas} 1=-322.5338252 \backslash \mathrm{MP} 2 / \mathrm{GTBas} 2=-322.4271759 \backslash \mathrm{MP} 4 / \mathrm{GTB}$ s $2=-322.532$ $186 \backslash \mathrm{MP} 2 / \mathrm{GTBas} 3=-322.6118981 \backslash \mathrm{MP} 4 / \mathrm{GTBas} 3=-322.7269283 \backslash \mathrm{HF} / \mathrm{GTLargeXP}=-321$. $5759722 \backslash \mathrm{MP} 2 / \mathrm{GTL}$ argeXP $=-323.0740327 \backslash \mathrm{HF} / \mathrm{GFHFB} 1=-321.600905 \backslash \mathrm{HF} / \mathrm{GFHFB} 2=-32$ $1.6052365 \backslash G 4=-323.2760411 \backslash$ FreqCoord $=-5.6740284005,5.8411195468,-1.0073$ $237575,-7.5721991708,4.131627165,-1.8453023056,-3.3514041794,4.9222134$ $579,0.1424772395,-2.9365677829,2.3719937675,0.4464634246,-7.0193766491$ $, 1.6573260742,-1.4784014266,-4.8027329222,0.6574577343,-0.3759869765,-$ $1.9637361865,6.302972643,0.7496710831,-4.5497404735,-1.3699042711,-0.1$ $710851793,-1.2077149955,1.6835863043,1.302503785,-9.3056590335,4.80814$ $0793,-2.7030564738,-6.0020361217,8.4592276342,-1.2725536414,-6.2615958$ $943,10.6409133794,-1.4869815164 \backslash \mathrm{PG}=\mathrm{C} 01 \quad[\mathrm{X}(\mathrm{C} 7 \mathrm{H} 4 \mathrm{N1})] \backslash \mathrm{NImag}=0 \backslash \backslash$

${ }^{3}$ o-cyanophenyl cation

$\backslash \backslash 1,3 \backslash \mathrm{C}, 0,-2.7679275795,2.8685033676,-0.3366486961 \backslash \mathrm{C}, 0,-3.9877217799$

$, 2.1239729746,-0.2468758638 \backslash \mathrm{C}, 0,-1.5650900384,2.1396088317,-0.54595214$ $32 \backslash \mathrm{C}, 0,-1.6429621186,0.7708293803,-0.6492919523 \backslash \mathrm{C}, 0,-4.1083870865,0.77$ $39160072,-0.3433644358 \backslash \mathrm{C}, 0,-2.9013941568,0.0813841873,-0.5501815391 \backslash \mathrm{C}$, $0,-2.7824983813,4.2645473141,-0.2195965187 \backslash \mathrm{H}, 0,-0.6220624396,2.6690324$ $406,-0.6191675108 \backslash \mathrm{H}, 0,-5.0582761657,0.2563970295,-0.2683154342 \backslash \mathrm{H}, 0,-2$. $9127303872,-1.0016422602,-0.6381813762 \backslash \mathrm{H}, 0,-0.7435950795,0.1861379867$, $-0.809065922 \backslash \mathrm{N}, 0,-2.789644788,5.4288127413,-0.1225986171 \backslash \backslash$ Version=EM64 L-G0 9RevB.01 \State=3-A \MP2/GTBas $1=-322.4173183 \backslash \mathrm{MP} 4 / \mathrm{GTB}$ as $1=-322.5214769$ $\backslash \mathrm{CCSD}(\mathrm{T}) / \mathrm{GBBas} 1=-322.5330577 \backslash \mathrm{MP} 2 / \mathrm{GTBas} 2=-322.4277975 \backslash \mathrm{MP} 4 / \mathrm{GTB}$ s $2=-322.5$ $324998 \backslash \mathrm{MP} 2 / \mathrm{GTBas} 3=-322$. 61243 \MP4/GTBas3=-322.7271258\HF/GTLargeXP=-321 $.5750138 \backslash \mathrm{MP} 2 / \mathrm{GTLargeXP}=-323.0744901 \backslash \mathrm{HF} / \mathrm{GFHFB} 1=-321.5999793 \backslash \mathrm{HF} / \mathrm{GFHFB} 2=-$ $321.6043099 \backslash \mathrm{G} 4=-323.2748763 \backslash$ FreqCoord $=-5.2306250809,5.420685776,-0.636$ $1738386,-7.5357020582,4.0137272355,-0.4665277708,-2.957591546,4.043274$ $7235,-1.0317000323,-3.1047484508,1.456656424,-1.2269839702,-7.76372644$ $15,1.4624893035,-0.6488647474,-5.4828403599,0.1537938256,-1.0396924323$ 
$,-5.2581599058,8.0588265044,-0.4149772797,-1.1755276485,5.0437403525,-$ $1.1700570259,-9.5587566578,0.484520167,-0.5070426878,-5.5042627307,-1$. $892829555,-1.2059880241,-1.4051910541,0.3517498178,-1.528913016,-5.271$ $6646574,10.2589693078,-0.2316778106 \backslash \mathrm{PG}=\mathrm{C} 01 \quad[\mathrm{X}(\mathrm{C} 7 \mathrm{H} 4 \mathrm{~N} 1)] \backslash \mathrm{NImag}=0 \backslash \backslash$

${ }^{3}$ p-cyanophenyl cation

$\backslash \backslash 1,3 \backslash C, 0,-2.7557101386,2.9892263301,0 . \backslash C, 0,-3.9727065697,2.22094862$

$6,0 . \backslash C, 0,-1.5505746354,2.3289222573,0 . \backslash C, 0,-1.6226007602,0.9294469372$, $0 . \backslash C, 0,-3.9688335792,0.781740972,0 . \backslash C, 0,-2.7653680937,0.1183977315,0 . \backslash$ $\mathrm{C}, 0,-5.2004980576,2.8956802039,0 . \backslash \mathrm{H}, 0,-2.8136574177,4.0723853339,0 . \backslash \mathrm{H}$, $0,-0.6019473866,2.8544311678,0 . \backslash \mathrm{H}, 0,-4.9142581433,0.2499855185,0 . \backslash \mathrm{H}, 0$, $-2.700430572,-0.9641162722,0 . \backslash \mathrm{N}, 0,-6.2251046462,3.4587511941,0 . \backslash \backslash$ Versi on=EM6 4L-G0 9RevB.01 \State=3-A" \MP2/GTBas1=-322.4275431 \MP 4/GTBas $1=-322$ $.529721 \backslash \mathrm{CCSD}(\mathrm{T}) / \mathrm{G} 3 \mathrm{Bas} 1=-322.5385597 \backslash \mathrm{MP} 2 / \mathrm{GTBas} 2=-322.4381013 \backslash \mathrm{MP} 4 / \mathrm{GTBas} 2$ $=-322.5407484 \backslash \mathrm{MP} 2 / \mathrm{GTBas} 3=-322.6224139 \backslash \mathrm{MP} 4 / \mathrm{GTBas} 3=-322.7351303 \backslash \mathrm{HF} / \mathrm{GTLar}$ geXP $=-321.5797707 \backslash \mathrm{MP} 2 / \mathrm{GTLargeXP}=-323.0847708 \backslash \mathrm{HF} / \mathrm{GFHFB} 1=-321.6046925 \backslash \mathrm{HF}$ $/ \mathrm{GFHFB} 2=-321.6090195 \backslash \mathrm{G} 4=-323.279859 \backslash$ FreqCoord $=-5.2075374635,5.64881911$ $31,0 .,-7.507327423,4.1969846584,0 .,-2.9301614095,4.4010252511,0 .,-3.06$ $62710598,1.7564001663,0 \ldots,-7.5000085317,1.4772763439,0 .,-5.2257883536,0$ $.2237392873,0 .,-9.8275170835,5.4720425537,0 .,-5.3170419512,7.695692988$ $7,0 .,-1.1375157071,5.3940931724,0 .,-9.2866020371,0.4724041671,0 .,-5.10$ $3074222,-1.8219157148,0 .,-11.7637429298,6.5360925186,0 . \backslash P G=C S \quad[S G(C 7 H 4$ N1) ] \NImag=0 \\

${ }^{3}$ m-bromophenyl cation

$\backslash \backslash 1,3 \backslash \mathrm{C}, 0,-2.9972875559,2.8409104042,-0.4049144741 \backslash \mathrm{C}, 0,-3.985546950$

$7,1.9295589075,-0.8710978777 \backslash C, 0,-1.7558859414,2.3882365122,0.16452592$ $99 \backslash \mathrm{C}, 0,-1.4962362585,1.0398625063,0.2709505058 \backslash \mathrm{C}, 0,-3.6488744751,0.626$ $2503405,-0.7286025391 \backslash \mathrm{C}, 0,-2.4609245313,0.1126331239,-0.1844939799 \backslash \mathrm{H}, 0$ $,-1.0385577546,3.1261416345,0.5036777005 \backslash \mathrm{H}, 0,-2.2885685826,-0.95710240$ $25,-0.1156158908 \backslash \mathrm{H}, 0,-0.563758859,0.6888396907,0.6985738311 \backslash \mathrm{H}, 0,-4.919$ $0502834,2.2760554508,-1.2992415466 \backslash \mathrm{Br}, 0,-3.2925900977,4.6536238038,-0$. $5231288692 \backslash \backslash$ Version=EM64L-G0 9RevB.01 \State $=3-\mathrm{A} \backslash \mathrm{MP} 2 / \mathrm{GTBas} 1=-2802.235318$ $1 \backslash \mathrm{MP} 4 / \mathrm{GTBas} 1=-2802.3172443 \backslash \mathrm{CCSD}(\mathrm{T}) / \mathrm{G} 3 \mathrm{Bas} 1=-2802.3217237 \backslash \mathrm{MP} 2 / \mathrm{GTBas} 2=-28$ $02.2445092 \backslash \mathrm{MP} 4 / \mathrm{GTBas} 2=-2802.3268132 \backslash \mathrm{MP} 2 / \mathrm{GTBas} 3=-2802.423674 \backslash \mathrm{MP} 4 / \mathrm{GTBas} 3$ $=-2802.5200418 \backslash \mathrm{HF} / \mathrm{GTLargeXP}=-2801.6495616 \backslash \mathrm{MP} 2 / \mathrm{GTL}$ LrgeXP $=-2803.8851823 \backslash$ $\mathrm{HF} / \mathrm{GFHFB} 1=-2801.7319343 \backslash \mathrm{HF} / \mathrm{GFHFB} 2=-2801.7354142 \backslash \mathrm{G} 4=-2804.1168265 \backslash \mathrm{FreqC}$ oord $=-5.6640526222,5.3685426321,-0.7651774633,-7.5315922265,3.64633789$ $25,-1.6461364237,-3.3181435497,4.5131129485,0.3109089493,-2.8274767587$ $, 1.9650553527,0.5120222516,-6.8953734511,1.1834416342,-1.3768592587,-4$ $.6504733978,0.2128457577,-0.3486430951,-1.9625897294,5.9075515417,0.95$ $18129132,-4.3247678574,-1.8086614219,-0.2184823702,-1.0653498484,1.301$ $7183649,1.3201132243,-9.2956578696,4.3011214652,-2.4552107035,-6.22209$ $35526,8.7940745146,-0.988570295 \backslash \mathrm{PG}=\mathrm{C} 01 \quad[\mathrm{X}(\mathrm{C} 6 \mathrm{H} 4 \mathrm{Br} 1)] \backslash \mathrm{NImag}=0 \backslash \backslash$

${ }^{3}$ o-bromophenyl cation

$\backslash \backslash 1,3 \backslash C, 0,-2.7250849915,2.6169781049,-0.2873061122 \backslash C, 0,-3.941455641$

$6,1.9078462772,-0.2148939288 \backslash C, 0,-1.5063575339,1.882325774,-0.25096397$ $28 \backslash C, 0,-1.5843021471,0.5150942609,-0.146725477 \backslash C, 0,-4.0637029824,0.559$ $0516569,-0.1113533955 \backslash \mathrm{C}, 0,-2.8449843808,-0.1544419024,-0.0766057172 \backslash \mathrm{H}$, $0,-0.5575238075,2.4037027457,-0.3050541747 \backslash \mathrm{H}, 0,-5.0200740985,0.0510784$ $953,-0.0581554439 \backslash \mathrm{H}, 0,-2.8636599806,-1.2372990797,0.0052801946 \backslash \mathrm{H}, 0,-0$. $6767925353,-0.0774778646,-0.116233684 \backslash \mathrm{Br}, 0,-2.7486519111,4.4466914822$, $-0.424808947 \backslash \backslash$ Version=EM64L-G09RevB.01 \State=3-A \MP2/GTBas1=-2802.2337 $056 \backslash \mathrm{MP} 4 / \mathrm{GTBas} 1=-2802.316783 \backslash \mathrm{CCSD}(\mathrm{T}) / \mathrm{GBBas} 1=-2802.3222765 \backslash \mathrm{MP} 2 / \mathrm{GTBas} 2=-2$ $802.2430236 \backslash \mathrm{MP} 4 / \mathrm{GTBas} 2=-2802.326489 \backslash \mathrm{MP} 2 / \mathrm{GTBas} 3=-2802.4203749 \backslash \mathrm{MP} 4 / \mathrm{GTBas}$ $3=-2802.5183033 \backslash \mathrm{HF} / \mathrm{GTLargeXP}=-2801.6519812 \backslash \mathrm{MP} 2 / \mathrm{GTLargeXP}=-2803.8818202$ 
$\backslash \mathrm{HF} / \mathrm{GFHFB} 1=-2801.7344435 \backslash \mathrm{HF} / \mathrm{GFHFB} 2=-2801.7379229 \backslash \mathrm{G} 4=-2804.1160396 \backslash \mathrm{Freq}$ Coord $=-5.1496643228,4.945371914,-0.5429298684,-7.4482717275,3.60530696$ $76,-0.406090673,-2.8466031973,3.5570802057,-0.4742531777,-2.9938971698$ $, 0.9733870857,-0.2772709683,-7.6792857222,1.0564545256,-0.2104274215,-$ $5.3762413321,-0.2918528989,-0.1447638257,-1.0535673088,4.5423398942,-0$ $.5764688459,-9.486565213,0.0965243674,-0.1098978622,-5.411533101,-2.33$ $81564051,0.0099781217,-1.2789525404,-0.1464119454,-0.2196498302,-5.194$ $1993466,8.4030290989,-0.8027725686 \backslash \mathrm{PG}=\mathrm{C} 01 \quad[\mathrm{X}(\mathrm{C} 6 \mathrm{H} 4 \mathrm{Br} 1)] \backslash \mathrm{NImag}=0 \backslash \backslash$

${ }^{3}$ p-bromophenyl cation

$\backslash \backslash 1,3 \backslash \mathrm{C}, 0,-2.8241397621,3.0317485539,-0.2334398731 \backslash \mathrm{C}, 0,-4.018212340$

$6,2.2643958641,-0.0618321107 \backslash C, 0,-1.6764001918,2.3873513023,-0.6405116$ $858 \backslash \mathrm{C}, 0,-1.786168495,1.0108866699,-0.8535349177 \backslash \mathrm{C}, 0,-4.0687997176,0.85$ $48702725,-0.2958162126 \backslash \mathrm{C}, 0,-2.9212058502,0.2102129251,-0.7028963198 \backslash \mathrm{H}$, $0,-2.8399121236,4.0988363531,-0.0424427539 \backslash \mathrm{H}, 0,-0.7426429394,2.9191501$ $602,-0.7866680496 \backslash \mathrm{H}, 0,-5.0005552723,0.3199065184,-0.1507273578 \backslash \mathrm{H}, 0,-2$. $9016818962,-0.8569516379,-0.8948683456 \backslash \mathrm{Br}, 0,-5.5492514816,3.1242330983$ ,0.4812273018\\Version=EM64L-G09RevB.01 \State=3-A \MP2/GTBas $1=-2802.237$ $9875 \backslash \mathrm{MP} 4 / \mathrm{GTBas} 1=-2802.3208962 \backslash \mathrm{CCSD}(\mathrm{T}) / \mathrm{G} 3 \mathrm{Bas} 1=-2802.3263036 \backslash \mathrm{MP} 2 / \mathrm{GTB} a \mathrm{~s} 2=$ $-2802.2473677 \backslash \mathrm{MP} 4 / \mathrm{GTBas} 2=-2802.3306022 \backslash \mathrm{MP} 2 / \mathrm{GTB} a \mathrm{~s} 3=-2802.4250412 \backslash \mathrm{MP} 4 / \mathrm{GT}$ Bas $3=-2802.5227481 \backslash \mathrm{HF} / \mathrm{GTLargeXP}=-2801.6561141 \backslash \mathrm{MP} 2 / \mathrm{GTLargeXP}=-2803.8864$ $977 \backslash \mathrm{HF} / \mathrm{GFHFB} 1=-2801.7385639 \backslash \mathrm{HF} / \mathrm{GFHFB} 2=-2801.7420415 \backslash \mathrm{G} 4=-2804.1199987 \backslash \mathrm{F}$ reqCoord $=-5.3368507114,5.7291744706,-0.4411374287,-7.5933208674,4.2790$ $880395,-0.1168457555,-3.1679372515,4.5114401443,-1.210391671,-3.375369$ $2827,1.9102989575,-1.6129472392,-7.6889171559,1.6154706942,-0.55901162$ $75,-5.5202790347,0.397244858,-1.3282815442,-5.3666561551,7.7456781708$, $-0.0802051813,-1.40339177,5.5163943435,-1.4865871711,-9.4496799769,0.6$ $045357079,-0.284833427,-5.4833841087,-1.6194039048,-1.6910560981,-10.4$ $865655427,5.9039449311,0.9093878081 \backslash \mathrm{PG}=\mathrm{C} 01 \quad[\mathrm{X}(\mathrm{C} 6 \mathrm{H} 4 \mathrm{Br} 1)] \backslash \mathrm{NImag}=0 \backslash \backslash$

${ }^{3} \mathrm{~m}$-chlorophenyl cation

$\backslash \backslash 1,3 \backslash \mathrm{C}, 0,-3.0003081355,2.855712621,-0.4061591303 \backslash \mathrm{C}, 0,-3.9897122879$ $, 1.943222469,-0.8728829499 \backslash \mathrm{C}, 0,-1.7548545492,2.398680361,0.1651106301 \backslash$ $\mathrm{C}, 0,-1.4962098157,1.0521811239,0.2710894619 \backslash \mathrm{C}, 0,-3.6546537644,0.641010$ $3502,-0.7311227903 \backslash \mathrm{C}, 0,-2.4634031124,0.1260851778,-0.1855014905 \backslash \mathrm{H}, 0,-1$ $.0415498064,3.1418360525,0.5024548527 \backslash \mathrm{H}, 0,-2.2931286216,-0.9442963239$, $-0.1175924998 \backslash \mathrm{H}, 0,-0.5644643513,0.6990217321,0.6983523851 \backslash \mathrm{H}, 0,-4.92107$ $971,2.2972975204,-1.2999620924 \backslash \mathrm{Cl}, 0,-3.2679562997,4.5142589059,-0.5131$ $453943 \backslash \backslash$ Version=EM64L-G09RevB.01 \State=3-A \MP2/GTBas $1=-689.4792239 \backslash M P 4$ $/ \mathrm{GTBas} 1=-689.5721015 \backslash \mathrm{CCSD}(\mathrm{T}) / \mathrm{G} 3 \mathrm{Bas} 1=-689.5775887 \backslash \mathrm{MP} 2 / \mathrm{GTB}$ s $2=-689.48811$ $53 \backslash \mathrm{MP} 4 / \mathrm{GTBas} 2=-689.5814554 \backslash \mathrm{MP} 2 / \mathrm{GTBas} 3=-689.6793396 \backslash \mathrm{MP} 4 / \mathrm{GTB}$ s $3=-689.787$ $669 \backslash \mathrm{HF} / \mathrm{GTLargeXP}=-688.7640607 \backslash \mathrm{MP} 2 / \mathrm{GTLargeXP}=-690.3758139 \backslash \mathrm{HF} / \mathrm{GFHFB} 1=-68$ $8.7915775 \backslash \mathrm{HF} / \mathrm{GFHFB} 2=-688.7960981 \backslash \mathrm{G} 4=-690.5664886 \backslash$ FreqCoord $=-5.66976069$ $03,5.396514768,-0.7675295225,-7.5394635731,3.6721582817,-1.6495097214$, $-3.3161945011,4.5328489627,0.3120138727,-2.8274267891,1.9883341663,0.5$ $122848405,-6.9062947253,1.2113340103,-1.3816218432,-4.6551572374,0.238$ $2664555,-0.3505470144,-1.9682438878,5.9372096937,0.9495020658,-4.33338$ $50824,-1.7844614405,-0.2222176198,-1.0666830358,1.3209596346,1.3196947$ $52,-9.2994929301,4.3412631593,-2.4565723377,-6.1755424206,8.5307130251$ ,$-0.9697042616 \backslash \mathrm{PG}=\mathrm{C} 01 \quad[\mathrm{X}(\mathrm{C} 6 \mathrm{H} 4 \mathrm{Cl1})] \backslash \mathrm{NImag}=0 \backslash \backslash$

${ }^{3}$ o-chlorophenyl cation

$\backslash \backslash 1,3 \backslash \mathrm{C}, 0,-2.7248844069,2.633587877,-0.2885599595 \backslash \mathrm{C}, 0,-3.9475684052$ $, 1.9189419228,-0.2156327696 \backslash \mathrm{C}, 0,-1.5060734665,1.8962411273,-0.25201654$ $67 \backslash \mathrm{C}, 0,-1.5832907528,0.5307884428,-0.1479252504 \backslash \mathrm{C}, 0,-4.0665922866,0.57$ $14495427,-0.11224211 \backslash \mathrm{C}, 0,-2.8467509761,-0.1416677625,-0.0775423519 \backslash \mathrm{H}, 0$ $,-0.5599500553,2.4226661988,-0.3064441157 \backslash \mathrm{H}, 0,-5.0224799634,0.06226040$ 
$81,-0.0589599708 \backslash \mathrm{H}, 0,-2.8636798054,-1.2246357683,0.0043234225 \backslash \mathrm{H}, 0,-0.6$ $76196081,-0.0625025306,-0.1173739934 \backslash \mathrm{Cl}, 0,-2.7351237945,4.3064305724,-$ $0.4144459478 \backslash \backslash$ Version=EM64L-G09RevB.01 \State=3-A \MP2/GTBas $1=-689.47673$ $94 \backslash \mathrm{MP} 4 / \mathrm{GTBas} 1=-689.5706708 \backslash \mathrm{CCSD}(\mathrm{T}) / \mathrm{G} 3 \mathrm{Bas} 1=-689.5775003 \backslash \mathrm{MP} 2 / \mathrm{GTB} a \mathrm{~s} 2=-689$ $.4856153 \backslash \mathrm{MP} 4 / \mathrm{GTBas} 2=-689.5800325 \backslash \mathrm{MP} 2 / \mathrm{GTBas} 3=-689.6748101 \backslash \mathrm{MP} 4 / \mathrm{GTB} a \mathrm{~s} 3=-6$ $89.7847135 \backslash \mathrm{HF} / \mathrm{GTL}$ argeXP $=-688.7658966 \backslash \mathrm{MP} 2 / \mathrm{GTLargeXP}=-690.3710206 \backslash \mathrm{HF} / \mathrm{GFH}$ $\mathrm{FB} 1=-688.7934724 \backslash \mathrm{HF} / \mathrm{GFHFB} 2=-688.7980149 \backslash \mathrm{G} 4=-690.5645713 \backslash$ FreqCoord $=-5.1$ $492852728,4.9767598345,-0.5452992964,-7.4598231766,3.6262746991,-0.407$ $4868798,-2.8460663877,3.5833764126,-0.4762422542,-2.9919859116,1.00304$ $47914,-0.2795382113,-7.6847457157,1.0798831344,-0.2121068485,-5.379579$ $7134,-0.2677132729,-0.1465338088,-1.0581522526,4.5781756271,-0.5790954$ $536,-9.4911116387,0.1176551202,-0.1114181976,-5.4115705645,-2.31422621$ $46,0.0081700844,-1.2778254053,-0.1181126654,-0.2218047027,-5.168634911$ $1,8.1379743921,-0.7831893383 \backslash \mathrm{PG}=\mathrm{C} 01 \quad[\mathrm{X}(\mathrm{C} 6 \mathrm{H} 4 \mathrm{Cl} 1)] \backslash \mathrm{NImag}=0 \backslash \backslash$

${ }^{3} \mathrm{p}$-chlorophenyl cation

$\backslash \backslash 1,3 \backslash \mathrm{C}, 0,-2.8326978265,3.0402371075,-0.2297596631 \backslash \mathrm{C}, 0,-4.029004768$ $9,2.2704575968,-0.0580027878 \backslash \mathrm{C}, 0,-1.6869721917,2.3968236771,-0.6361443$ $176 \backslash \mathrm{C}, 0,-1.7983510726,1.0177295877,-0.8492151936 \backslash \mathrm{C}, 0,-4.0805426255,0.8$ $5778113,-0.2922950674 \backslash \mathrm{C}, 0,-2.9348361002,0.214332782,-0.6986814546 \backslash \mathrm{H}, 0$, $-2.8562744205,4.1069835907,-0.036821879 \backslash \mathrm{H}, 0,-0.751929565,2.9260364637$, $-0.7830842161 \backslash \mathrm{H}, 0,-5.0160145756,0.3296311071,-0.1450583426 \backslash \mathrm{H}, 0,-2.9124$ $077881,-0.8525953781,-0.8913559809 \backslash \mathrm{Cl}, 0,-5.429939095,3.0572217973,0.43$ $89096716 \backslash \backslash$ Version=EM64L-G09RevB.01 \State=3-A \MP2/GTBas1=-689.4817277\M $\mathrm{P} 4 / \mathrm{GTBas} 1=-689.5754852 \backslash \mathrm{CCSD}(\mathrm{T}) / \mathrm{G} 3 \mathrm{Bas} 1=-689.5823807 \backslash \mathrm{MP} 2 / \mathrm{GTBas} 2=-689.490$ $7106 \backslash \mathrm{MP} 4 / \mathrm{GTBas} 2=-689.5848914 \backslash \mathrm{MP} 2 / \mathrm{GTBas} 3=-689.6801788 \backslash \mathrm{MP} 4 / \mathrm{GTBas} 3=-689.7$ $898333 \backslash \mathrm{HF} / \mathrm{GTLargeXP}=-688.7707645 \backslash \mathrm{MP} 2 / \mathrm{GTLargeXP}=-690.3764995 \backslash \mathrm{HF} / \mathrm{GFHFB} 1=$ $-688.7983425 \backslash \mathrm{HF} / \mathrm{GFHFB} 2=-688.8028781 \backslash \mathrm{G} 4=-690.569447 \backslash \mathrm{FreqCoord}=-5.353023$ $1093,5.7452155123,-0.4341828396,-7.6137156013,4.2905430543,-0.10960938$ $4,-3.1879154361,4.5293403385,-1.2021385413,-3.3983910181,1.9232301982$, $-1.6047841438,-7.7111080357,1.6209714176,-0.5523576273,-5.5460364743,0$ $.4050302594,-1.3203166033,-5.397576415,7.7610742186,-0.0695832669,-1.4$ $20940949,5.5294075712,-1.4798147075,-9.4788938264,0.6229125173,-0.2741$ $205408,-5.5036531069,-1.6111717668,-1.6844186908,-10.2610978078,5.7773$ $119244,0.8294190764 \backslash \mathrm{PG}=\mathrm{C} 01 \quad[\mathrm{X}(\mathrm{C} 6 \mathrm{H} 4 \mathrm{Cl} 1)] \backslash \mathrm{NImag}=0 \backslash \backslash$

${ }^{3}$ m-ethynylphenyl cation

$\backslash \backslash 1,3 \backslash \mathrm{C}, 0,-2.9970339803,2.9141113105,-0.4037018608 \backslash \mathrm{C}, 0,-3.9870064951,1$ $.9799056066,-0.8720683059 \backslash \mathrm{C}, 0,-1.7604185492,2.4324056638,0.1640507876 \backslash$ $\mathrm{C}, 0,-1.5125569692,1.084089904,0.2647161659 \backslash \mathrm{C}, 0,-3.6660490042,0.6740435$ $341,-0.7370990354 \backslash \mathrm{C}, 0,-2.4840944191,0.1625639739,-0.1949753928 \backslash \mathrm{H}, 0,-1$. $0357826836,3.1613053814,0.5073548231 \backslash \mathrm{H}, 0,-2.3107184943,-0.907326538,-0$ $.1258907057 \backslash \mathrm{H}, 0,-0.5842451151,0.7214309253,0.6909132054 \backslash \mathrm{H}, 0,-4.9171771$ $631,2.3364820692,-1.2991894031 \backslash \mathrm{C}, 0,-3.2237669559,4.2770975493,-0.49443$ $16021 \backslash \mathrm{C}, 0,-3.4273794131,5.4783510116,-0.5761440963 \backslash \mathrm{H}, 0,-3.6053207578,6$ $.5300296084,-0.64756458 \backslash \backslash$ Version=EM64L-G09RevB. $01 \backslash$ State=3-A $\backslash \mathrm{MP} 2 / \mathrm{GTB} a \mathrm{~s} 1$ $=-306.3700344 \backslash \mathrm{MP} 4 / \mathrm{GTBas} 1=-306.473869 \backslash \mathrm{CCSD}(\mathrm{T}) / \mathrm{G} 3 \mathrm{Bas} 1=-306.4841926 \backslash \mathrm{MP} 2 / \mathrm{G}$ TBas2=-306.380869 \MP4/GTBas2=-306.4849149\MP2/GTBas3=-306.5683147\MP $/$ GTBas3 $=-306.6821104 \backslash \mathrm{HF} / \mathrm{GTLargeXP}=-305.5441312 \backslash \mathrm{MP} 2 / \mathrm{GTLargeXP}=-307.02102$ $82 \backslash \mathrm{HF} / \mathrm{GFHFB} 1=-305.5681542 \backslash \mathrm{HF} / \mathrm{GFHFB} 2=-305.5723233 \backslash \mathrm{G} 4=-307.2067264 \backslash \mathrm{FreqC}$ oord $=-5.6635734338,5.5068722975,-0.7628859562,-7.5343503658,3.74147936$ $54,-1.6479702673,-3.3267089372,4.5965805486,0.3100110604,-2.8583184322$ $, 2.0486330221,0.5002410565,-6.9278286076,1.273757681,-1.3929153096,-4$. $6942581402,0.3072013897,-0.368450095,-1.9573456052,5.9740013933,0.9587$ $616679,-4.3666251244,-1.7145986699,-0.2378989565,-1.104063262,1.363306$ $8726,1.3056367399,-9.2921181852,4.4153112252,-2.4551121666,-6.09203666$ $29,8.0825430119,-0.9343403193,-6.4768084443,10.3525830717,-1.088754555$ 
$1,-6.8130688534,12.3399675996,-1.2237197096 \backslash \mathrm{PG}=\mathrm{C} 01 \quad[\mathrm{X}(\mathrm{C} 8 \mathrm{H} 5)] \backslash \mathrm{NImag}=0 \backslash \backslash$

${ }^{3}$ o-ethynylphenyl cation

$\backslash \backslash 1,3 \backslash \mathrm{C}, 0,-2.7054447853,2.7289280084,-0.2589200825 \backslash \mathrm{C}, 0,-3.9289286347,2$ $.0070671557,-0.1971395713 \backslash \mathrm{C}, 0,-1.49470799,1.9546226694,-0.2368485245 \backslash \mathrm{C}$ $, 0,-1.5788339154,0.5884290547,-0.1591204012 \backslash \mathrm{C}, 0,-4.0537144156,0.658158$ $8872,-0.1196558519 \backslash \mathrm{C}, 0,-2.841298598,-0.0661868758,-0.1004764417 \backslash \mathrm{H}, 0,-0$ $.5419976347,2.4694356034,-0.2822456275 \backslash \mathrm{H}, 0,-5.0123198027,0.153805794,-$ $0.0746921654 \backslash \mathrm{H}, 0,-2.8726207803,-1.150229879,-0.0394066111 \backslash \mathrm{H}, 0,-0.67623$ $05376,-0.0113972758,-0.1414355191 \backslash \mathrm{C}, 0,-2.6911573949,4.109901941,-0.336$ $1848394 \backslash \mathrm{C}, 0,-2.676086387,5.3287221069,-0.4044243136 \backslash \mathrm{H}, 0,-2.6662791239$, $6.39610281,-0.464110051 \backslash \backslash$ Version=EM64L-G09RevB. $01 \backslash$ state=3-A \MP2/GTBas 1 $=-306.3644076 \backslash \mathrm{MP} 4 / \mathrm{GTBas} 1=-306.4700208 \backslash \mathrm{CCSD}(\mathrm{T}) / \mathrm{G} 3 \mathrm{Bas} 1=-306.4842825 \backslash \mathrm{MP} 2 /$ GTBas2 $=-306.3755684 \backslash \mathrm{MP} 4 / \mathrm{GTBas} 2=-306.4813515 \backslash \mathrm{MP} 2 / \mathrm{GTBas} 3=-306.5609666 \backslash \mathrm{MP}$ $4 / \mathrm{GTBas} 3=-306.677126 \backslash \mathrm{HF} / \mathrm{GTLargeXP}=-305.5496794 \backslash \mathrm{MP} 2 / \mathrm{GTLargeXP}=-307.0140$ $65 \backslash \mathrm{HF} / \mathrm{GFHFB} 1=-305.5737478 \backslash \mathrm{HF} / \mathrm{GFHFB} 2=-305.5779243 \backslash \mathrm{G} 4=-307.2058993 \backslash \mathrm{FreqC}$ oord $=-5.1125497119,5.1569265723,-0.4892880461,-7.4245991152,3.79280725$ $45,-0.3725397997,-2.8245887497,3.6937015382,-0.4475788464,-2.983563709$ $4,1.111969762,-0.3006939804,-7.6604100663,1.2437400487,-0.2261167902,-$ $5.369276212,-0.1250750689,-0.1898729577,-1.0242270942,4.6665569932,-0$. $5333669381,-9.4719117176,0.2906508283,-0.1411477368,-5.4284665584,-2.1$ $736194612,-0.0744677027,-1.2778905188,-0.0215377298,-0.2672743965,-5.0$ $855504569,7.7665891016,-0.6352972765,-5.0570703793,10.0698254203,-0.76$ $42511941,-5.0385373379,12.0868826286,-0.8770408918 \backslash \mathrm{PG}=\mathrm{C} 01 \quad[\mathrm{X}(\mathrm{C} 8 \mathrm{H} 5)] \backslash \mathrm{NI}$ $\operatorname{mag}=0 \backslash \backslash$

${ }^{3}$ p-ethynylphenyl cation

$\backslash \backslash 1,3 \backslash \mathrm{C}, 0,-2.9446984141,3.0784583139,-0.211275812 \backslash \mathrm{C}, 0,-4.1497903145,2$. $3085204707,-0.0534138118 \backslash \mathrm{C}, 0,-1.7804927329,2.447474277,-0.5874365683 \backslash \mathrm{C}$ $, 0,-1.8708808438,1.0708633047,-0.7901490079 \backslash \mathrm{C}, 0,-4.1573060577,0.888185$ $6738,-0.2827002478 \backslash \mathrm{C}, 0,-2.9941929887,0.2552246922,-0.6589259115 \backslash \mathrm{H}, 0,-2$ $.9768129233,4.1469799552,-0.0298940044 \backslash \mathrm{H}, 0,-0.8500100775,2.9884028476$, $-0.7180336965 \backslash \mathrm{H}, 0,-5.0842091926,0.3404980026,-0.1540240428 \backslash \mathrm{H}, 0,-2.9543$ $996187,-0.8126523804,-0.8419879692 \backslash \mathrm{C}, 0,-5.3207690833,2.9444862846,0.32$ $51619835 \backslash \mathrm{C}, 0,-6.3526339226,3.5049915044,0.6587406574 \backslash \mathrm{H}, 0,-7.2561838304$

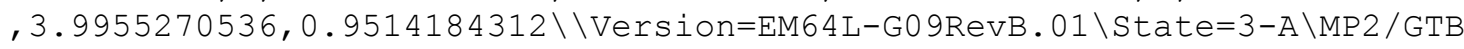
$\mathrm{as} 1=-306.3703293 \backslash \mathrm{MP} 4 / \mathrm{GTBas} 1=-306.4752615 \backslash \mathrm{CCSD}(\mathrm{T}) / \mathrm{G} 3 \mathrm{Bas} 1=-306.48892 \backslash \mathrm{MP} 2$ $/ \mathrm{GTBas} 2=-306.3814728 \backslash \mathrm{MP} 4 / \mathrm{GTBas} 2=-306.4865398 \backslash \mathrm{MP} 2 / \mathrm{GTBas} 3=-306.5671409 \backslash \mathrm{M}$ $\mathrm{P} 4 / \mathrm{GTBas} 3=-306.6825312 \backslash \mathrm{HF} / \mathrm{GTLargeXP}=-305.5542138 \backslash \mathrm{MP} 2 / \mathrm{GTLargeXP}=-307.02$ $03779 \backslash \mathrm{HF} / \mathrm{GFHFB} 1=-305.5782541 \backslash \mathrm{HF} / \mathrm{GFHFB} 2=-305.5824273 \backslash \mathrm{G} 4=-307.2106024 \backslash \mathrm{Fr}$ eqCoord $=-5.5646735465,5.8174431248,-0.3992534231,-7.8419672033,4.36247$ $14618,-0.1009374761,-3.3646436467,4.6250561008,-1.1100942345,-3.535452$ $422,2.0236383717,-1.4931652291,-7.8561698995,1.6784276787,-0.534226046$ $,-5.6582047376,0.4823047707,-1.2451895145,-5.6253611739,7.8366563939,-$ $0.0564914813,-1.6062862567,5.6472629567,-1.3568870405,-9.6077629762,0$. $6434479736,-0.2910632588,-5.5830061665,-1.5356904403,-1.5911266691,-10$ $.0547963839,5.56427268,0.6144670977,-12.0047383362,6.6234740414,1.2448$ $394351,-13.7122002094,7.5504518878,1.7979202728 \backslash \mathrm{PG}=\mathrm{C} 01 \quad[\mathrm{X}(\mathrm{C} 8 \mathrm{H} 5)] \backslash \mathrm{NImag}$ $=0 \backslash \backslash$

${ }^{3} \mathrm{~m}-($ dimethylamino) phenyl cation

$\backslash \backslash 1,3 \backslash \mathrm{C}, 0,-2.8818900279,2.6365199841,-0.4811291899 \backslash \mathrm{C}, 0,-3.915419656$

$1,1.677506334,-0.7662706988 \backslash \mathrm{C}, 0,-1.6567606372,2.167366454,0.0987478769$ $\backslash \mathrm{C}, 0,-1.4657056107,0.8315980666,0.3782654589 \backslash \mathrm{C}, 0,-3.6475300731,0.38837$ $27061,-0.4506984857 \backslash \mathrm{C}, 0,-2.4828823386,-0.1093472698,0.1118739795 \backslash \mathrm{H}, 0,-$ $0.8510887545,2.8619051034,0.290978321 \backslash \mathrm{H}, 0,-2.3505187532,-1.1612281299$, $0.3397254507 \backslash \mathrm{H}, 0,-0.5255275205,0.5003898035,0.8051010268 \backslash \mathrm{N}, 0,-3.059378$ 
$7871,3.9490882506,-0.7605791801 \backslash \mathrm{C}, 0,-2.1244436197,4.9878523101,-0.3135$ $575492 \backslash \mathrm{C}, 0,-4.2095854622,4.4299348631,-1.5336241739 \backslash \mathrm{H}, 0,-1.6830858723$, $4.7288428621,0.6467427163 \backslash \mathrm{H}, 0,-2.6773307123,5.9213500425,-0.2046052602$ $\backslash \mathrm{H}, 0,-1.3358581414,5.1327039657,-1.0610697756 \backslash \mathrm{H}, 0,-5.0693847327,4.5887$ $495072,-0.8723713172 \backslash \mathrm{H}, 0,-4.4722548714,3.7214677476,-2.3177759559 \backslash \mathrm{H}, 0$, $-3.9421239508,5.3809063367,-1.9940162594 \backslash \mathrm{H}, 0,-4.8697604784,1.975651062$ $1,-1.180166984 \backslash \backslash$ Version=EM64L-G09RevB.01 \State=3-A \MP2/GTBas1=-364.012 $5946 \backslash \mathrm{MP} 4 / \mathrm{GTBas} 1=-364.1412844 \backslash \mathrm{CCSD}(\mathrm{T}) / \mathrm{G} 3 \mathrm{Bas} 1=-364.1573955 \backslash \mathrm{MP} 2 / \mathrm{GTBas} 2=-3$ $64.0266298 \backslash \mathrm{MP} 4 / \mathrm{GTB} a \mathrm{~s} 2=-364.1562024 \backslash \mathrm{MP} 2 / \mathrm{GTBas} 3=-364.2828032 \backslash \mathrm{MP} 4 / \mathrm{GTB}$ as $3=$ $-364.4268231 \backslash \mathrm{HF} / \mathrm{GTLargeXP}=-363.0266059 \backslash \mathrm{MP} 2 / \mathrm{GTLargeXP}=-364.8144031 \backslash \mathrm{HF} / \mathrm{G}$ $\mathrm{FHFB} 1=-363.05509 \backslash \mathrm{HF} / \mathrm{GFHFB} 2=-363.0604736 \backslash \mathrm{G} 4=-365.0144713 \backslash \mathrm{FreqCoord}=-5.4$ $459828978,4.9823007139,-0.9092024035,-7.3990708454,3.1700275574,-1.448$ $0417645,-3.130823872,4.0957290277,0.1866064435,-2.7697821957,1.5714925$ $986,0.7148181228,-6.8928328996,0.7339180521,-0.8516967065,-4.691967640$ $2,-0.2066363933,0.2114111826,-1.6083246607,5.4082168637,0.5498693372,-$ $4.4418367137,-2.1944031432,0.6419880622,-0.993103089,0.9455996883,1.52$ $142045,-5.7813880444,7.4626952682,-1.4372863528,-4.014616626,9.4256748$ $575,-0.5925378949,-7.9549636566,8.3713636778,-2.8981296795,-3.18057135$ $67,8.9362179349,1.2221666122,-5.0594218134,11.1897299173,-0.386647907$, $-2.5244060396,9.6994048164,-2.0051312837,-9.5797488071,8.6714798611,-1$ $.6485428757,-8.4513369034,7.0325548554,-4.379961794,-7.4495346488,10.1$ $684393231,-3.7681446348,-9.2025136369,3.7334394415,-2.2301923908 \backslash P G=C 0$ $1[\mathrm{X}(\mathrm{C} 8 \mathrm{H} 10 \mathrm{~N} 1)] \backslash \mathrm{NImag}=0 \backslash \backslash$

${ }^{3}$ o- (dimethylamino) phenyl cation

$\backslash \backslash 1,3 \backslash \mathrm{C}, 0,-2.8436053931,2.6618553733,-0.4812453253 \backslash \mathrm{C}, 0,-3.833152054$

$6,1.6871069003,-0.739032293 \backslash \mathrm{C}, 0,-1.6398226991,2.1803541473,0.134411282$ $1 \backslash \mathrm{C}, 0,-1.5180291438,0.8433985821,0.4301133166 \backslash \mathrm{C}, 0,-3.7486798466,0.3604$ $508649,-0.4615658383 \backslash \mathrm{C}, 0,-2.5517138053,-0.0751723897,0.1427871729 \backslash \mathrm{H}, 0$, $-0.8335760133,2.8654091677,0.3641909211 \backslash \mathrm{H}, 0,-4.5495664158,-0.334932063$ $4,-0.6870906802 \backslash \mathrm{H}, 0,-2.4260951889,-1.1244394001,0.3880128643 \backslash \mathrm{H}, 0,-0.60$ $7535125,0.4835770797,0.894972408 \backslash \mathrm{N}, 0,-3.036029551,3.9555218252,-0.8001$ $335753 \backslash \mathrm{C}, 0,-2.0175460871,4.9764562333,-0.5379276017 \backslash \mathrm{C}, 0,-4.2752458183$, $4.4141345314,-1.4272310798 \backslash \mathrm{H}, 0,-1.8049160611,5.0334176875,0.5332615118$ $\backslash \mathrm{H}, 0,-2.3948617725,5.9392629579,-0.8776119158 \backslash \mathrm{H}, 0,-1.0971880752,4.7417$ $122553,-1.0799159055 \backslash \mathrm{H}, 0,-4.9541740517,3.5752364521,-1.5736306021 \backslash \mathrm{H}, 0$, $-4.0388901458,4.875068781,-2.3915906331 \backslash \mathrm{H}, 0,-4.7445727518,5.1658110143$ ,$-0.7845640267 \backslash \backslash$ Version=EM64L-G09RevB.01 State=3-A \MP2/GTBas $1=-364.013$ $0815 \backslash \mathrm{MP} 4 / \mathrm{GTBas} 1=-364.1431608 \backslash \mathrm{CCSD}(\mathrm{T}) / \mathrm{G} 3 \mathrm{Bas} 1=-364.1649959 \backslash \mathrm{MP} 2 / \mathrm{GTBas} 2=-3$ $64.0264015 \backslash \mathrm{MP} 4 / \mathrm{GTBas} 2=-364.1575111 \backslash \mathrm{MP} 2 / \mathrm{GTB} a \mathrm{~s} 3=-364.2819796 \backslash \mathrm{MP} 4 / \mathrm{GTB} a \mathrm{~s} 3=$ $-364.4278059 \backslash \mathrm{HF} / \mathrm{GTLargeXP}=-363.0482162 \backslash \mathrm{MP} 2 / \mathrm{GTLargeXP}=-364.8126179 \backslash \mathrm{HF} / \mathrm{G}$ $\mathrm{FHFB} 1=-363.0767232 \backslash \mathrm{HF} / \mathrm{GFHFB} 2=-363.082117 \backslash \mathrm{G} 4=-365.0204662 \backslash \mathrm{FreqCoord}=-5$. $3736354229,5.0301776609,-0.9094218675,-7.2436076088,3.1881699985,-1.39$ $65686372,-3.0988158079,4.120272211,0.2540005124,-2.8686593436,1.593792$ $3411,0.8127963745,-7.0839782699,0.681153419,-0.8722330267,-4.822040261$ $5,-0.1420552293,0.269828652,-1.5752303761,5.4148385856,0.6882211009,-8$ $.5974345493,-0.632929873,-1.298413214,-4.5846554794,-2.1248825192,0.73$ $32380496,-1.1480750023,0.9138282447,1.6912527476,-5.7372643827,7.47485$ $29623,-1.512033327,-3.812609565,9.4041393932,-1.0165358464,-8.07904374$ $74,8.3415053781,-2.6970758692,-3.4107970482,9.5117809418,1.0077182145$, $-4.5256328762,11.2235804217,-1.6584461718,-2.0733849784,8.9605375635,-$ $2.040745308,-9.3620321723,6.7562177548,-2.9737308723,-7.6323962564,9.2$ $12544875,-4.5194513186,-8.9659431185,9.7619680712,-1.4826111442 \backslash \mathrm{PG}=\mathrm{C} 01$ $[\mathrm{X}(\mathrm{C} 8 \mathrm{H} 10 \mathrm{~N} 1)] \backslash \mathrm{NImag}=0 \backslash \backslash$

${ }^{3} \mathrm{p}-($ dimethylamino) phenyl cation

$\backslash \backslash 1,3 \backslash \mathrm{C}, 0,-2.8494507091,2.9962350692,-0.1146935006 \backslash \mathrm{C}, 0,-4.076937252$ 
$7,2.2562810798,-0.0458632757 \backslash \mathrm{C}, 0,-1.6645173323,2.352031974,-0.40809327$ $14 \backslash \mathrm{C}, 0,-1.7326570704,0.9865462053,-0.6394614961 \backslash \mathrm{C}, 0,-4.060822657,0.842$ $7834068,-0.2921961415 \backslash \mathrm{C}, 0,-2.876437428,0.2037081783,-0.5987320543 \backslash \mathrm{H}, 0$, $-2.837261887,4.0561075065,0.1009686787 \backslash \mathrm{H}, 0,-0.7279249254,2.8967383603$, $-0.4440230328 \backslash \mathrm{H}, 0,-4.9813108422,0.2748792522,-0.2780199268 \backslash \mathrm{H}, 0,-2.8543$ $684185,-0.8602542006,-0.8053863506 \backslash \mathrm{N}, 0,-5.238336782,2.8852067411,0.248$ $4109117 \backslash \mathrm{C}, 0,-5.3502186963,4.3476730629,0.2962163748 \backslash \mathrm{C}, 0,-6.4759247455$, $2.1532093742,0.542008306 \backslash \mathrm{H}, 0,-7.0130440236,1.9281197512,-0.3865352246 \backslash$ $\mathrm{H}, 0,-7.1078670061,2.7851910948,1.1660764406 \backslash \mathrm{H}, 0,-6.2638355525,1.231359$ $0799,1.080772785 \backslash \mathrm{H}, 0,-4.7140958649,4.8111627677,-0.4558071587 \backslash \mathrm{H}, 0,-5.0$ $803731715,4.716515323,1.2924724691 \backslash \mathrm{H}, 0,-6.3862356352,4.6188359734,0.09$ $28054671 \backslash \backslash$ Version=EM6 4L-G09RevB.01 \State=3-A \MP2/GTBas1=-364.0094572\M $\mathrm{P} 4 / \mathrm{GTBas} 1=-364.1391176 \backslash \mathrm{CCSD}(\mathrm{T}) / \mathrm{G} 3 \mathrm{Bas} 1=-364.1608323 \backslash \mathrm{MP} 2 / \mathrm{GTB}$ as $2=-364.023$ $0466 \backslash \mathrm{MP} 4 / \mathrm{GTBas} 2=-364.1537549 \backslash \mathrm{MP} 2 / \mathrm{GTBas} 3=-364.2787227 \backslash \mathrm{MP} 4 / \mathrm{GTB}$ s $3=-364.4$ $240717 \backslash \mathrm{HF} / \mathrm{GTLargeXP}=-363.0433013 \backslash \mathrm{MP} 2 / \mathrm{GTLargeXP}=-364.809745 \backslash \mathrm{HF} / \mathrm{GFHFB} 1=-$ $363.0717802 \backslash \mathrm{HF} / \mathrm{GFHFB} 2=-363.0771675 \backslash \mathrm{G} 4=-365.0165908 \backslash \mathrm{FreqCoord}=-5.384681$ $4693,5.6620637105,-0.2167393054,-7.7042948685,4.2637533197,-0.08666903$ $07,-3.1454819015,4.4446962866,-0.7711845197,-3.2742473452,1.8643021455$ $,-1.2084071001,-7.6738426959,1.5926298281,-0.5521706845,-5.4356789772$, $0.384952668,-1.1314396097,-5.3616479337,7.6649323528,0.1908031508,-1.3$ $755787543,5.4740421795,-0.8390819286,-9.4133132744,0.5194465062,-0.525$ $3815212,-5.3939745933,-1.6256448438,-1.5219596338,-9.8990219098,5.4522$ $505775,0.4694285916,-10.110448087,8.2159114042,0.5597678245,-12.237724$ $2261,4.0689760241,1.02424726,-13.2527325625,3.6436182811,-0.7304457151$ $,-13.4319220306,5.263248397,2.2035651228,-11.8369337356,2.3269314323,2$ $.0423645756,-8.9083501488,9.0917800118,-0.8613506994,-9.600513947,8.91$ $29222619,2.4424190009,-12.0682363705,8.7283350424,0.1753769165 \backslash P G=C 01$ $[\mathrm{X}(\mathrm{C} 8 \mathrm{H} 10 \mathrm{~N} 1)] \backslash \mathrm{NImag}=0 \backslash \backslash$

${ }^{3}$ syn-m-thiomethoxyphenyl cation

$\backslash \backslash 1,3 \backslash \mathrm{C}, 0,0.3075302504,1.2114394396,0.0000221904 \backslash \mathrm{C}, 0,1.6467976482,1.4$ $270000391,0.0001038764 \backslash \mathrm{C}, 0,2.6510366872,0.4644922094,0.0001139566 \backslash \mathrm{C}, 0$, $2.2477750728,-0.8846594831,0.0000048338 \backslash \mathrm{C}, 0,0.9020671516,-1.194450014$, $-0.0000864373 \backslash \mathrm{C}, 0,-0.087046425,-0.1581285315,-0.0000762197 \backslash \mathrm{H}, 0,-0.4138$ $930038,2.0184436445,-0.000016554 \backslash \mathrm{H}, 0,3.7017488917,0.7354250415,0.00019$ $30725 \backslash \mathrm{H}, 0,2.9904122817,-1.6744383463,-0.0000037942 \backslash \mathrm{H}, 0,0.5793404108,-2$ $.2302336047,-0.0001746545 \backslash S, 0,-1.7049756354,-0.7206126683,-0.000229934$ $9 \backslash \mathrm{C}, 0,-2.7661826184,0.7461483559,0.000108013 \backslash \mathrm{H}, 0,-2.5931697238,1.33709$ $44828,-0.901645403 \backslash \mathrm{H}, 0,-3.7862647723,0.3586019594,-0.0008349811 \backslash \mathrm{H}, 0,-2$ $.5943882159,1.3358674757,0.9028910207 \backslash \backslash V e r s i o n=E M 64 L-G 09 R e v B .01 \backslash$ State= $3-\mathrm{A} \backslash \mathrm{MP} 2 / \mathrm{GTBas} 1=-667.2873088 \backslash \mathrm{MP} 4 / \mathrm{GTBas} 1=-667.3993 \backslash \mathrm{CCSD}(\mathrm{T}) / \mathrm{G} 3 \mathrm{Bas} 1=-667.4$ $073003 \backslash \mathrm{MP} 2 / \mathrm{GTBas} 2=-667.2988361 \backslash \mathrm{MP} 4 / \mathrm{GTBas} 2=-667.4114118 \backslash \mathrm{MP} 2 / \mathrm{GTB}$ s $3=-667$ $.5261463 \backslash \mathrm{MP} 4 / \mathrm{GTBas} 3=-667.6543784 \backslash \mathrm{HF} / \mathrm{GTLargeXP}=-666.4557515 \backslash \mathrm{MP} 2 / \mathrm{GTLarge}$ $\mathrm{XP}=-668.2712175 \backslash \mathrm{HF} / \mathrm{GFHFB} 1=-666.4837572 \backslash \mathrm{HF} / \mathrm{GFHFB} 2=-666.489634 \backslash \mathrm{G} 4=-668.4$ $696783 \backslash$ FreqCoord=0 .5811479509,2.2892887674,0.0000419337,3.1119965514,2 $.6966392655,0.0001962979,5.009733307,0.8777630665,0.0002153468,4.24767$ $92959,-1.6717641439,0.0000091346,1.7046598701,-2.257183406,-0.00016334$ $29,-0.1644939041,-0.2988196183,-0.0001440344,-0.7821444255,3.814305702$ $8,-0.0000312825,6.995291618,1.3897519197,0.0003648542,5.6510602369,-3$. $1642299009,-0.00000717,1.094794714,-4.2145307253,-0.0003300493,-3.2219$ $370141,-1.361760591,-0.0004345139,-5.2273275823,1.4100160472,0.0002041$ $15,-4.900380594,2.5267423863,-1.7038628806,-7.1550034862,0.677659494,-$ $0.0015778857,-4.9026832104,2.524423679,1.706216757 \backslash \mathrm{PG}=\mathrm{C} 01 \quad[\mathrm{X}(\mathrm{C} 7 \mathrm{H} 7 \mathrm{~S} 1)] \backslash$ NImag $=0 \backslash \backslash$

${ }^{3}$ syn-o-thiomethoxyphenyl cation

$\backslash \backslash 1,3 \backslash \mathrm{C}, 0,0.8345047317,-1.2864220919,0.0000123339 \backslash \mathrm{C}, 0,-0.144356751,-0$ 
$.238942352,0.0000671438 \backslash \mathrm{C}, 0,0.3301072595,1.0785482291,0.0001348146 \backslash \mathrm{C}, 0$ $, 1.6372819608,1.4508807519,0.0001502544 \backslash \mathrm{C}, 0,2.5802651046,0.397883163,0$ $.0000992712 \backslash \mathrm{C}, 0,2.1690533194,-0.9524126298,0.0000285945 \backslash \mathrm{H}, 0,0.51113260$ $7,-2.3223986486,-0.0000437328 \backslash \mathrm{H}, 0,1.9551470203,2.4874514809,0.00019737$ $4 \backslash \mathrm{H}, 0,3.6391504199,0.6364869313,0.0001089865 \backslash \mathrm{H}, 0,2.9185065331,-1.73511$ $45894,-0.0000141067 \backslash \mathrm{S}, 0,-1.7944293416,-0.673330563,0.0000295561 \backslash \mathrm{C}, 0,-2$ $.6918101192,0.9025379238,0.0001495506 \backslash \mathrm{H}, 0,-2.440833381,1.4670880997,-0$ $.8995160431 \backslash \mathrm{H}, 0,-3.7495033318,0.6355699826,-0.0000829776 \backslash \mathrm{H}, 0,-2.441161$ $0317,1.4667143127,0.9001469807 \backslash \backslash$ Version=EM64L-G0 9RevB.01 \State=3-A \MP2 $/ \mathrm{GTBas} 1=-667.276298 \backslash \mathrm{MP} 4 / \mathrm{GTBas} 1=-667.392156 \backslash \mathrm{CCSD}(\mathrm{T}) / \mathrm{G} 3 \mathrm{Bas} 1=-667.4108577$ $\backslash \mathrm{MP} 2 / \mathrm{GTBas} 2=-667.2879257 \backslash \mathrm{MP} 4 / \mathrm{GTBas} 2=-667.4045132 \backslash \mathrm{MP} 2 / \mathrm{GTB} a \mathrm{~s} 3=-667.51413$ $88 \backslash \mathrm{MP} 4 / \mathrm{GTBas} 3=-667.6465286 \backslash \mathrm{HF} / \mathrm{GTLargeXP}=-666.4718762 \backslash \mathrm{MP} 2 / \mathrm{GTLargeXP}=-66$ $8.2587951 \backslash \mathrm{HF} / \mathrm{GFHFB} 1=-666.4998751 \backslash \mathrm{HF} / \mathrm{GFHFB} 2=-666.5057704 \backslash \mathrm{G} 4=-668.472425$ $1 \backslash$ FreqCoord=1.5769853995,-2.430985445,0.0000233077,-0.2727947249,-0.45 $15356069,0.0001268835,0.6238123149,2.0381607741,0.0002547626,3.0940145$ $082,2.7417672725,0.0002839396,4.875994398,0.7518902109,0.0001875953,4$. $0989167413,-1.7997990359,0.0000540358,0.9659006449,-4.3886974173,-0.00$ $0082643,3.6946924179,4.7006020678,0.0003729828,6.87699765,1.2027859873$ $, 0.0002059547,5.5151780646,-3.278891383,-0.0000266579,-3.3909800204,-1$ $.272410361,0.0000558529,-5.086783927,1.7055495004,0.0002826096,-4.6125$ $066262,2.7723947212,-1.6998389737,-7.0855344314,1.2010532054,-0.000156$ $805,-4.6131257962,2.7716883661,1.7010312729 \backslash \mathrm{PG}=\mathrm{C} 01 \quad[\mathrm{X}(\mathrm{C} 7 \mathrm{H} 7 \mathrm{~S} 1)] \backslash \mathrm{NImag}=0$ $\backslash 1$

${ }^{3} \mathrm{p}$-thiomethoxyphenyl cation

$\backslash \backslash 1,3 \backslash \mathrm{C}, 0,0.3633432208,1.1638364492,0.0000018944 \backslash \mathrm{C}, 0,1.713602606,1.45$

$92189447,0.0000022231 \backslash \mathrm{C}, 0,2.5896614445,0.3797593135,0.0000010999 \backslash \mathrm{C}, 0,2$ $.259523274,-0.9661698808,-0.0000003076 \backslash \mathrm{C}, 0,0.9092258847,-1.2609194506$, $-0.0000006257 \backslash \mathrm{C}, 0,-0.0503564944,-0.1979638336,0.0000004001 \backslash \mathrm{H}, 0,-0.3664$ $737745,1.9634020684,0.0000029534 \backslash \mathrm{H}, 0,2.0658119597,2.4847451511,0.00000$ $33758 \backslash \mathrm{H}, 0,3.0093491247,-1.7490179998,-0.0000010813 \backslash \mathrm{H}, 0,0.5668559045,-2$ $.2912092425,-0.0000016646 \backslash \mathrm{S}, 0,-1.6788584794,-0.7214129372,-0.000000141$ $8 \backslash \mathrm{C}, 0,-2.7178343672,0.7626800438,-0.000001911 \backslash \mathrm{H}, 0,-2.5398564287,1.3503$ $098464,-0.9026205665 \backslash \mathrm{H}, 0,-3.7423729911,0.386806099,0.0000024353 \backslash \mathrm{H}, 0,-2$ $.5398508838,1.3503154284,0.9026119168 \backslash \backslash$ Version=EM64L-G09RevB.01 \State= 3-A \MP2 / GTBas $1=-667.2771984 \backslash \mathrm{MP} 4 / \mathrm{GTBas} 1=-667.3922043 \backslash \mathrm{CCSD}(\mathrm{T}) / \mathrm{G} 3 \mathrm{Bas} 1=-66$ $7.4096659 \backslash \mathrm{MP} 2 / \mathrm{GTBas} 2=-667.2885071 \backslash \mathrm{MP} 4 / \mathrm{GTBas} 2=-667.40423 \backslash \mathrm{MP} 2 / \mathrm{GTBas} 3=-66$ $7.5158629 \backslash \mathrm{MP} 4 / \mathrm{GTBas} 3=-667.6473103 \backslash \mathrm{HF} / \mathrm{GTLargeXP}=-666.4705029 \backslash \mathrm{MP} 2 / \mathrm{GTLarg}$ $\mathrm{eXP}=-668.2605845 \backslash \mathrm{HF} / \mathrm{GFHFB} 1=-666.4985191 \backslash \mathrm{HF} / \mathrm{GFHFB} 2=-666.5044198 \backslash \mathrm{G} 4=-668$ $.4716747 \backslash$ FreqCoord=0.6866191795,2.1993321524,0.0000035798,3.238239626, $2.7575241734,0.0000042011,4.8937509071,0.7176410989,0.0000020785,4.269$ $8801788,-1.8257964726,-0.0000005813,1.718187915,-2.3827924372,-0.00000$ $11825,-0.0951599835,-0.3740974297,0.000000756,-0.6925350687,3.71029219$ $8,0.0000055811,3.9038188459,4.6954878456,0.0000063793,5.686845684,-3.3$ $05165021,-0.0000020433,1.0712024163,-4.3297579815,-0.0000031457,-3.172$ $582742,-1.36327288,-0.0000002681,-5.1359626286,1.4412564098,-0.0000036$ $113,-4.799633067,2.5517158042,-1.7057056726,-7.0720600402,0.7309575936$ $, 0.000004602,-4.7996225887,2.5517263528,1.705689327 \backslash \mathrm{PG}=\mathrm{C} 01 \quad[\mathrm{X}(\mathrm{C} 7 \mathrm{H} 7 \mathrm{~S} 1)]$ $\backslash \mathrm{NImag}=0 \backslash \backslash$

${ }^{3}$ syn-m-methoxyphenyl cation

$\backslash \backslash 1,3 \backslash \mathrm{C}, 0,-1.2704218276,1.4026830375,0.0000255209 \backslash \mathrm{C}, 0,0.0599309301,1$. $1720673711,0.0000249552 \backslash \mathrm{C}, 0,0.4238038553,-0.2128437795,-0.0000027432 \backslash \mathrm{C}$ $, 0,-0.5929251056,-1.2414738177,-0.0000208332 \backslash \mathrm{C}, 0,-1.9268748398,-0.9103$ $458421,-0.0000218542 \backslash \mathrm{C}, 0,-2.3027417038,0.4508867037,0.0000070044 \backslash \mathrm{H}, 0,0$ $.8010368747,1.9615609077,0.0000520437 \backslash \mathrm{H}, 0,-0.2529041908,-2.2705527983$, $-0.0000400679 \backslash \mathrm{H}, 0,-2.6871476092,-1.6830024939,-0.0000427722 \backslash \mathrm{H}, 0,-3.346$ 
$4724642,0.7476504653,-0.0000000214 \backslash 0,0,1.6445206657,-0.6642497762,-0.0$ $000099716 \backslash \mathrm{C}, 0,2.797942928,0.2203226727,-0.0000414702 \backslash \mathrm{H}, 0,2.784235361,0$ $.8354678446,0.9022524242 \backslash \mathrm{H}, 0,3.6588918218,-0.4438924346,-0.0002059901 \backslash$ $\mathrm{H}, 0,2.7840503043,0.8356799398,-0.9021862244 \backslash \backslash$ Version=EM64L-G09RevB .01 State $=3-\mathrm{A} \backslash \mathrm{MP} 2 / \mathrm{GTBas} 1=-344.6686239 \backslash \mathrm{MP} 4 / \mathrm{GTBas} 1=-344.7732942 \backslash \mathrm{CCSD}(\mathrm{T}) / \mathrm{G} 3 \mathrm{Ba}$ $\mathrm{s} 1=-344.778605 \backslash \mathrm{MP} 2 / \mathrm{GTB} a \mathrm{~s} 2=-344.6814173 \backslash \mathrm{MP} 4 / \mathrm{GTB} a \mathrm{~s} 2=-344.7870675 \backslash \mathrm{MP} 2 / \mathrm{GTB}$ as $3=-344.909892 \backslash \mathrm{MP} 4 / \mathrm{GTBas} 3=-345.0283887 \backslash \mathrm{HF} / \mathrm{GTLargeXP}=-343.7899929 \backslash \mathrm{MP} 2 /$ GTLargeXP $=-345.3943668 \backslash \mathrm{HF} / \mathrm{GFHFB} 1=-343.8174548 \backslash \mathrm{HF} / \mathrm{GFHFB} 2=-343.8226882 \backslash \mathrm{G}$ $4=-345.5759532 \backslash$ FreqCoord $=-2.4007493274,2.650686792,0.0000482275,0.1132$ $530448,2.2148863407,0.0000471585,0.8008732206,-0.4022164523,-0.0000051$ $84,-1.120466067,-2.3460455167,-0.000039369,-3.6412657395,-1.7203043277$ $,-0.0000412985,-4.3515511749,0.8520523869,0.0000132365,1.5137403156,3$. $7068129085,0.0000983483,-0.4779196584,-4.290722959,-0.0000757174,-5.07$ $79730601,-3.1804137944,-0.0000808278,-6.3239164685,1.4128546225,-0.000$ $0000404,3.1076936781,-1.2552501609,-0.0000188436,5.2873458693,0.416349$ $5122,-0.0000783673,5.2614423219,1.5788054191,1.7050099845,6.914303493$, $-0.8388351339,-0.0003892649,5.2610926152,1.5792062209,-1.704884885 \backslash P G=$ $\mathrm{C} 01[\mathrm{X}(\mathrm{C} 7 \mathrm{H} 7 \mathrm{O} 1)] \backslash \mathrm{NImag}=0 \backslash \backslash$

\footnotetext{
${ }^{3}$ syn-o-methoxyphenyl cation

$\backslash \backslash 1,3 \backslash \mathrm{C}, 0,-1.2888139833,1.4275274467,-0.0006786727 \backslash \mathrm{C}, 0,0.0148128486,1$ $.0672088517,-0.0012472669 \backslash \mathrm{C}, 0,0.4883194497,-0.27119726,-0.0008103408 \backslash \mathrm{C}$ $, 0,-0.4941928464,-1.3242836077,0.0003625948 \backslash \mathrm{C}, 0,-1.8215014662,-0.99194$ $20537,0.000964146 \backslash \mathrm{C}, 0,-2.2359320288,0.367626256,0.0005215453 \backslash \mathrm{H}, 0,-1.61$ $39010831,2.4624731529,-0.0004620114 \backslash \mathrm{H}, 0,-0.140613594,-2.3494965387,0.0$ $004437735 \backslash \mathrm{H}, 0,-2.5748882408,-1.7710246897,0.00169012 \backslash \mathrm{H}, 0,-3.2950026002$ $, 0.6056262458,0.0014821169 \backslash 0,0,1.73601705,-0.6150007574,-0.0018342466 \backslash$ $\mathrm{C}, 0,2.7846205378,0.3997366476,0.0020718807 \backslash \mathrm{H}, 0,2.7326534484,0.95607944$ $19,0.9401392763 \backslash \mathrm{H}, 0,3.7148625762,-0.1581821155,-0.0717709384 \backslash \mathrm{H}, 0,2.648$ 9839322,1.0601719799,-0.8564139768\\Version=EM64L-G0 9RevB.01\State=3-A $\backslash \mathrm{MP} 2 / \mathrm{GTBas} 1=-344.6602029 \backslash \mathrm{MP} 4 / \mathrm{GTBas} 1=-344.76772 \backslash \mathrm{CCSD}(\mathrm{T}) / \mathrm{G} 3 \mathrm{Bas} 1=-344.780$ $4869 \backslash \mathrm{MP} 2 / \mathrm{GTBas} 2=-344.6728815 \backslash \mathrm{MP} 4 / \mathrm{GTBas} 2=-344.7814847 \backslash \mathrm{MP} 2 / \mathrm{GTBas} 3=-344.9$ $004036 \backslash \mathrm{MP} 4 / \mathrm{GTBas} 3=-345.0222572 \backslash \mathrm{HF} / \mathrm{GTLargeXP}=-343.7996181 \backslash \mathrm{MP} 2 / \mathrm{GTLargeXP}$ $=-345.3842901 \backslash \mathrm{HF} / \mathrm{GFHFB} 1=-343.8271326 \backslash \mathrm{HF} / \mathrm{GFHFB} 2=-343.8323704 \backslash \mathrm{G} 4=-345.57$ $70882 \backslash$ FreqCoord $=-2.4355054647,2.6976359215,-0.0012825055,0.0279922271$, $2.0167324564,-0.0023569928,0.9227900254,-0.5124885493,-0.0015313221,-0$ $.9338891366,-2.5025333408,0.0006852048,-3.4421389218,-1.8744988212,0.0$ $018219719,-4.2252991862,0.6947129431,0.0009855778,-3.0498310527,4.6533$ $998687,-0.0008730751,-0.2657211833,-4.4399050083,0.0008386104,-4.86583$ $35979,-3.3467516381,0.0031938639,-6.2266525216,1.1444677435,0.00280079$ $51,3.2805967865,-1.1621830029,-0.0034662237,5.2621702004,0.7553927892$, $0.003915287,5.1639666336,1.8067283065,1.776605759,7.0200728903,-0.2989$ $208775,-0.1356274178,5.0058541622,2.0034346958,-1.6183878726 \backslash P G=C 01] \mathrm{X}$ (C7H701) ] \NImag=1 \\
}

${ }^{3} \mathrm{p}$-methoxyphenyl cation

$\backslash \backslash 1,3 \backslash \mathrm{C}, 0,-1.3438663267,1.4404249642,0.0000961923 \backslash \mathrm{C}, 0,-0.0043188821,1$ $.1297155964,0.0000789405 \backslash \mathrm{C}, 0,0.3790529627,-0.2518522057,0.0000141976 \backslash \mathrm{C}$ $, 0,-0.6027572824,-1.307339517,-0.0000240038 \backslash \mathrm{C}, 0,-1.9399196721,-0.99077$ $0196,-0.0000108441 \backslash \mathrm{C}, 0,-2.2449752406,0.368911185,0.0000442487 \backslash \mathrm{H}, 0,-1.6$ $872225868,2.4690555238,0.0001544433 \backslash \mathrm{H}, 0,0.7468549147,1.9091076751,0.00$ $01392183 \backslash \mathrm{H}, 0,-0.242745317,-2.3302306027,-0.0000677747 \backslash \mathrm{H}, 0,-2.708942214$ $8,-1.7547645923,-0.0000416415 \backslash 0,0,1.6122295453,-0.6692583765,-0.000011$ $0906 \backslash \mathrm{C}, 0,2.7498063153,0.2362167274,-0.0000813619 \backslash \mathrm{H}, 0,2.7277940008,0.85$ $03883631,0.902477003 \backslash \mathrm{H}, 0,3.6212327219,-0.4142504695,-0.000351668 \backslash \mathrm{H}, 0,2$ $.7274730617,0.8507149246,-0.9024048591 \backslash \backslash$ Version=EM64L-G09RevB.01 \State $=3-\mathrm{A} \backslash \mathrm{MP} 2 / \mathrm{GTBas} 1=-344.6639848 \backslash \mathrm{MP} 4 / \mathrm{GTBas} 1=-344.7706359 \backslash \mathrm{CCSD}(\mathrm{T}) / \mathrm{G} 3 \mathrm{Bas} 1=-3$ 
$44.7821295 \backslash \mathrm{MP} 2 / \mathrm{GTBas} 2=-344.6766859 \backslash \mathrm{MP} 4 / \mathrm{GTB} a \mathrm{~s} 2=-344.7843587 \backslash \mathrm{MP} 2 / \mathrm{GTB}$ as $3=$ $-344.9040881 \backslash \mathrm{MP} 4 / \mathrm{GTBas} 3=-345.025 \backslash \mathrm{HF} / \mathrm{GTLargeXP}=-343.8008883 \backslash \mathrm{MP} 2 / \mathrm{GTLarge}$ $\mathrm{XP}=-345.3884148 \backslash \mathrm{HF} / \mathrm{GFHFB} 1=-343.8283739 \backslash \mathrm{HF} / \mathrm{GFHFB} 2=-343.833614 \backslash \mathrm{G} 4=-345.5$ $782287 \backslash$ FreqCoord=-2.5395393166,2.7220086973,0.0001817771, -0.0081615044 ,2.1348530853,0.0001491759,0.7163062894,-0.4759316947,0.0000268296,-1. $1390461884,-2.4705136498,-0.0000453606,-3.6659169001,-1.8722843311,-0$. $0000204925,-4.2423883798,0.6971411071,0.000083618,-3.1883886143,4.6658$ $387468,0.0002918556,1.4113512499,3.6076906641,0.0002630845,-0.45872216$ $92,-4.4034976656,-0.0001280757,-5.1191588958,-3.3160245071,-0.00007869$ $1,3.0466723041,-1.2647150438,-0.0000209582,5.1963808545,0.4463849228,-$ $0.0001537517,5.1547836085,1.6070011128,1.7054343769,6.8431381078,-0.78$ $28199378,-0.0006645563,5.1541771215,1.6076182247,-1.7052980446 \backslash \mathrm{PG}=\mathrm{C} 01$ $[\mathrm{X}(\mathrm{C} 7 \mathrm{H} 701)] \backslash \mathrm{NImag}=0 \backslash \backslash$

${ }^{3}$ anti-o-thiomethoxyphenyl cation

$\backslash \backslash 1,3 \backslash \mathrm{C}, 0,-3.5477748017,3.67499296,-0.0031344862 \backslash \mathrm{C}, 0,-4.7187823172,2$. $9520133171,-0.0026070616 \backslash \mathrm{C}, 0,-2.3066696198,2.9712064846,-0.0008367655 \backslash$ $\mathrm{C}, 0,-4.7139312344,1.5358003231,0.0001604167 \backslash \mathrm{C}, 0,-3.506428744,0.8129580$ $573,0.0024863056 \backslash \mathrm{C}, 0,-2.3720372915,1.5651610607,0.0018646686 \backslash \mathrm{H}, 0,-5.65$ $71588979,0.9987210573,0.0005077697 \backslash \mathrm{S}, 0,-0.7375510484,3.6455581069,-0.0$ $00963906 \backslash \mathrm{H}, 0,-3.5677352821,4.7577376173,-0.0052729305 \backslash \mathrm{H}, 0,-5.668736346$ $, 3.4740986844,-0.0043420144 \backslash \mathrm{C}, 0,-0.9611950066,5.4431196581,-0.00449332$ $19 \backslash \mathrm{H}, 0,0.0495844995,5.8543995328,-0.0050898512 \backslash \mathrm{H}, 0,-1.4898199453,5.759$ $5551846,0.8968894137 \backslash \mathrm{H}, 0,-1.4894528106,5.7560292392,-0.9073264606 \backslash \mathrm{H}, 0$, $-3.4940311541,-0.2709912833,0.0046182236 \backslash \backslash$ Version=EM64L-G09RevB.01 \Sta te $=3-\mathrm{A} \backslash \mathrm{MP} 2 / \mathrm{GTBas} 1=-667.2746303 \backslash \mathrm{MP} 4 / \mathrm{GTBas} 1=-667.3903399 \backslash \mathrm{CCSD}(\mathrm{T}) / \mathrm{G} 3 \mathrm{Bas} 1=$ $-667.4086795 \backslash \mathrm{MP} 2 / \mathrm{GTBas} 2=-667.2861416 \backslash \mathrm{MP} 4 / \mathrm{GTBas} 2=-667.4025701 \backslash \mathrm{MP} 2 / \mathrm{GTB} a \mathrm{~s}$ $3=-667.5125238 \backslash \mathrm{MP} 4 / \mathrm{GTBas} 3=-667.6447394 \backslash \mathrm{HF} / \mathrm{GTLargeXP}=-666.4694001 \backslash \mathrm{MP} 2 / \mathrm{G}$ TLargeXP $=-668.257285 \backslash \mathrm{HF} / \mathrm{GFHFB} 1=-666.4973883 \backslash \mathrm{HF} / \mathrm{GFHFB} 2=-666.5033019 \backslash \mathrm{G} 4=$ $-668.4701603 \backslash$ FreqCoord=-6.7043227564,6.9447302347,-0.0059233205,-8.917 $2062603,5.5784967099,-0.0049266324,-4.3589738604,5.6147665401,-0.00158$ $12576,-8.9080390422,2.9022420054,0.0003031436,-6.6261900307,1.53626808$ $57,0.0046984367,-4.4825008579,2.9577257586,0.003523713,-10.6904810072$, $1.8873092814,0.0009595457,-1.3937694906,6.8891064236,-0.0018215183,-6$. $7420425979,8.9908211087,-0.0099643946,-10.7123592135,6.5650950721,-0.0$ $082052181,-1.8163953228,10.2860054624,-0.0084911478,0.0937011245,11.06$ $32117896,-0.0096184248,-2.815351684,10.8839819461,1.6948753634,-2.8146$ $578999,10.8773188749,-1.7145985237,-6.602761981,-0.5120993098,0.008727$ $1779 \backslash \mathrm{PG}=\mathrm{C} 01 \quad[\mathrm{X}(\mathrm{C} 7 \mathrm{H} 7 \mathrm{~S} 1)] \backslash \mathrm{NImag}=0 \backslash \backslash$

3anti-o-methoxyphenyl cation

$\backslash \backslash 1,3 \backslash \mathrm{C}, 0,-1.2500679472,1.4308532971,0.0035302566 \backslash \mathrm{C}, 0,0.0340144859,0$. $9940708909,0.0004857897 \backslash \mathrm{C}, 0,0.4489433614,-0.370690994,-0.0003813411 \backslash \mathrm{C}$, $0,-0.580194037,-1.3676197916,0.0023885172 \backslash \mathrm{C}, 0,-1.8873749001,-0.9567903$ $882,0.0055522247 \backslash \mathrm{C}, 0,-2.2408984567,0.4265390673,0.0061184449 \backslash \mathrm{H}, 0,-1.51$ $26478034,2.4830026961,0.0039821685 \backslash \mathrm{H}, 0,-0.3245588785,-2.4198464949,0.0$ $021504107 \backslash \mathrm{H}, 0,-2.6813144177,-1.6951044541,0.007743915 \backslash \mathrm{H}, 0,-3.289678783$ $1,0.7061993473,0.0086102208 \backslash 0,0,1.7273883014,-0.5931516034,-0.00347763$ $93 \backslash \mathrm{C}, 0,2.2770699083,-1.9393014738,-0.0053013063 \backslash \mathrm{H}, 0,1.959604734,-2.463$ $0050452,0.8989295104 \backslash \mathrm{H}, 0,3.3551101677,-1.7976300354,-0.0106095447 \backslash \mathrm{H}, 0$, $1.950874265,-2.4645050181,-0.9055116272 \backslash \backslash$ Version=EM64L-G09RevB. $01 \backslash$ Stat $\mathrm{e}=3-\mathrm{A} \backslash \mathrm{MP} 2 / \mathrm{GTBas} 1=-344.658335 \backslash \mathrm{MP} 4 / \mathrm{GTBas} 1=-344.7652442 \backslash \mathrm{CCSD}(\mathrm{T}) / \mathrm{G} 3 \mathrm{Bas} 1=-3$ $44.7768677 \backslash \mathrm{MP} 2 / \mathrm{GTBas} 2=-344.6711463 \backslash \mathrm{MP} 4 / \mathrm{GTBas} 2=-344.7790765 \backslash \mathrm{MP} 2 / \mathrm{GTB} a \mathrm{~s} 3=$ $-344.8985617 \backslash \mathrm{MP} 4 / \mathrm{GTBas} 3=-345.0197891 \backslash \mathrm{HF} / \mathrm{GTLargeXP}=-343.7963651 \backslash \mathrm{MP} 2 / \mathrm{GTL}$ $\operatorname{argeXP}=-345.382908 \backslash \mathrm{HF} / \mathrm{GFHFB} 1=-343.823879 \backslash \mathrm{HF} / \mathrm{GFHFB} 2=-343.829123 \backslash \mathrm{G} 4=-345$ $.5734214 \backslash$ FreqCoord $=-2.3622860678,2.7039208678,0.0066712182,0.064278062$ $8,1.8785217405,0.0009180094,0.8483800022,-0.7005044586,-0.0007206302,-$ 
$1.0964078338,-2.58442686,0.0045136434,-3.5666216713,-1.8080718002,0.01$ $0492184,-4.2346843748,0.8060420222,0.0115621853,-2.858490084,4.6921950$ $828,0.0075252078,-0.6133273944,-4.572847159,0.0040636874,-5.0669499256$ $,-3.2032831849,0.0146338786,-6.2165919651,1.3345233616,0.0162709593,3$. $2642908147,-1.1208940856,-0.0065717859,4.3030385122,-3.6647486745,-0.0$ $10018017,3.703116276,-4.6544049994,1.6987305874,6.3402393626,-3.397028$ $4551,-0.0200491338,3.6866180805,-4.6572395374,-1.7111689855 \backslash P G=C 01 \quad[X($ $\mathrm{C} 7 \mathrm{H} 7 \mathrm{O} 1)] \backslash \mathrm{NImag}=0 \backslash \backslash$

\section{${ }^{3}$ p-hydroxyphenyl cation}

$\backslash \backslash 1,3 \backslash C, 0,-2.6834472279,2.602186985,0 . \backslash C, 0,-3.9265771605,1.953708977$ $9,0 . \backslash \mathrm{C}, 0,-1.4157157273,2.0143116634,0 . \backslash \mathrm{C}, 0,-1.3854555742,0.6409849249$, $0 . \backslash C, 0,-3.8993922807,0.5809525208,0 . \backslash C, 0,-2.6275869341,-0.0833585719,0$ $. \backslash \mathrm{H}, 0,-4.8584504751,2.5081383232,0 . \backslash \mathrm{H}, 0,-0.50836871,2.6077546034,0 . \backslash \mathrm{H}$, $0,-0.4613482819,0.0732689844,0 . \backslash \mathrm{H}, 0,-4.8135562759,-0.0056950005,0 . \backslash 0,0$ $,-2.5189405142,-1.3856127569,0 . \backslash \mathrm{H}, 0,-3.3736808382,-1.8491806536,0 . \backslash \backslash \mathrm{Ve}$ rsion=EM64L-G09RevB.01 \State=3-A" \MP2/GTBas1=-305.5013103\MP 4/GTBas $1=-$ $305.5903824 \backslash \mathrm{CCSD}(\mathrm{T}) / \mathrm{G} 3 \mathrm{Bas} 1=-305.601278 \backslash \mathrm{MP} 2 / \mathrm{GTBas} 2=-305.5126858 \backslash \mathrm{MP} 4 / \mathrm{GTB}$ as $2=-305.6025104 \backslash \mathrm{MP} 2 / \mathrm{GTBas} 3=-305.7085974 \backslash \mathrm{MP} 4 / \mathrm{GTBas} 3=-305.8092475 \backslash \mathrm{HF} / \mathrm{GT}$ LargeXP $=-304.7585398 \backslash \mathrm{MP} 2 / \mathrm{GTLargeXP}=-306.133549 \backslash \mathrm{HF} / \mathrm{GFHFB} 1=-304.7836046 \backslash$ $\mathrm{HF} / \mathrm{GFHFB} 2=-304.7883031 \backslash \mathrm{G} 4=-306.3052028 \backslash$ FreqCoord $=-5.0709803527,4.91742$ $07481,0 .,-7.420155473,3.6919749116,0 .,-2.6753150067,3.80649739,0 .,-2.6$ $181316046,1.2112859633,0 .,-7.3687834952,1.0978411606,0 .,-4.9654196958$, $-0.1575248717,0 .,-9.1811408282,4.7396945342,0 .,-0.9606776364,4.9279420$ $223,0 .,-0.8718219046,0.1384583145,0 .,-9.0963030867,-0.0107619912,0 .,-4$ $.7601077168,-2.6184286368,0 .,-6.3753328439,-3.4944450056,0 . \backslash P G=C S \quad[S G($ $\mathrm{C} 6 \mathrm{H} 5 \mathrm{O} 1)] \backslash \mathrm{NImag}=0 \backslash \backslash$

${ }^{3}$ p-thiolphenyl cation

$\backslash \backslash 1,3 \backslash C, 0,-2.6885709496,2.6562661799,0 . \backslash C, 0,-3.9198507757,2.00610874$

$16,0 . \backslash C, 0,-1.4257825568,2.074889088,0 . \backslash C, 0,-1.3909585618,0.6957058759$, $0 . \backslash C, 0,-3.885123976,0.6274740376,0 . \backslash C, 0,-2.6205614463,-0.0394367137,0$. $\backslash \mathrm{H}, 0,-4.858031641,2.5498461327,0 . \backslash \mathrm{H}, 0,-0.5171100431,2.6663054398,0 . \backslash \mathrm{H}$, $0,-0.4452748396,0.1626838567,0 . \backslash \mathrm{H}, 0,-4.8039928292,0.0508638019,0 . \backslash \mathrm{S}, 0$, $-2.4763490209,-1.7438754129,0 . \backslash \mathrm{H}, 0,-3.7927433601,-2.0504510275,0 . \backslash \backslash$ Ver sion=EM64L-G0 9RevB.01 State=3-A" \MP2/GTBas $1=-628.10056 \backslash \mathrm{MP} 4 / \mathrm{GTB}$ s $1=-628$ $.1995657 \backslash \mathrm{CCSD}(\mathrm{T}) / \mathrm{GBBas} 1=-628.2131394 \backslash \mathrm{MP} 2 / \mathrm{GTBas} 2=-628.1104215 \backslash \mathrm{MP} 4 / \mathrm{GTBas}$ $2=-628.209886 \backslash \mathrm{MP} 2 / \mathrm{GTBas} 3=-628.3027036 \backslash \mathrm{MP} 4 / \mathrm{GTBas} 3=-628.4156948 \backslash \mathrm{HF} / \mathrm{GTLar}$ geXP $=-627.4101402 \backslash \mathrm{MP} 2 / \mathrm{GTLargeXP}=-628.9878679 \backslash \mathrm{HF} / \mathrm{GFHFB} 1=-627.4351966 \backslash \mathrm{HF}$ /GFHFB2 $=-627.440345 \backslash G 4=-629.1798992 \backslash$ FreqCoord $=-5.0806627835,5.01961561$ $6,0 .,-7.4074444479,3.7909961144,0 .,-2.6943385574,3.9209721324,0 .,-2.62$ $8530744,1.3146935745,0 .,-7.341820307,1.1857540866,0 .,-4.9521434479,-0$. $0745245885,0 .,-9.1803493464,4.8185108719,0 .,-0.9771963619,5.0385870679$ $, 0 .,-0.8414475007,0.3074279354,0 .,-9.0782307915,0.0961186557,0 .,-4.679$ $621459,-3.2954469403,0 .,-7.1672462428,-3.8747908909,0 . \backslash P G=C S \quad[S G(C 6 H 5 S$ 1) ] \NImag=0\\

\section{${ }^{3}$ m-nitrophenyl cation}

$\backslash \backslash 1,3 \backslash C, 0,-2.7107659461,2.9045334061,0 . \backslash C, 0,-3.928793144,2.1760852$ $73,0 . \backslash C, 0,-1.4966209152,2.2718079135,0 . \backslash C, 0,-1.5789912209,0.8596837435$ $, 0 . \backslash \mathrm{C}, 0,-3.9293824073,0.7423587872,0 . \backslash \mathrm{C}, 0,-2.7321300329,0.060734172,0$. $\backslash \mathrm{N}, 0,-2.7711910887,4.3933919312,0 . \backslash \mathrm{H}, 0,-4.859303834,2.7349930325,0 . \backslash \mathrm{H}$, $0,-4.8791419512,0.2173927281,0 . \backslash \mathrm{H}, 0,-2.6734565868,-1.0222870024,0 . \backslash \mathrm{H}, 0$ $,-0.5596191096,2.8194271666,0 . \backslash 0,0,-1.7096787462,4.977187523,0 . \backslash 0,0,-3$ $.8934650172,4.8730113258,0 . \backslash \backslash$ Version=EM64L-G0 9RevB.01 \State=3-A" \MP2/G TBas1=-434.4294999\MP 4/GTBas1=-434.530462 \CCSD $(\mathrm{T}) / \mathrm{G} 3 \mathrm{Bas} 1=-434.5249324 \backslash$ MP2 / GTBas2 $=-434.4493038 \backslash \mathrm{MP} 4 / \mathrm{GTBas} 2=-434.5518245 \backslash \mathrm{MP} 2 / \mathrm{GTBas} 3=-434.705093$ 
$5 \backslash \mathrm{MP} 4 / \mathrm{GTBas} 3=-434.8221234 \backslash \mathrm{HF} / \mathrm{GTL}$ LrgeXP $=-433.353034 \backslash \mathrm{MP} 2 / \mathrm{GTLargeXP}=-435$. $2802543 \backslash \mathrm{HF} / \mathrm{GFHFB} 1=-433.3882779 \backslash \mathrm{HF} / \mathrm{GFHFB} 2=-433.3950172 \backslash \mathrm{G} 4=-435.5052869 \backslash$ FreqCoord $=-5.1226052486,5.4887726813,0 .,-7.4243430749,4.1122052078,0 .$, $-2.8282036544,4.293094783,0 .,-2.9838609737,1.6245668361,0 .,-7.42545662$ $12,1.4028548002,0 .,-5.1629775216,0.1147709521,0 .,-5.2367922196,8.30230$ $75445,0 .,-9.1827534427,5.1683878067,0 .,-9.2202420512,0.4108127193,0 .,-$ $5.0521007772,-1.9318424638,0 .,-1.0575268559,5.3279451965,0,-3.2308246$ $055,9.4055213305,0 .,-7.3575825904,9.2086568481,0 . \backslash P G=C S \quad[S G(C 6 H 4 N 102)]$ $\backslash N \operatorname{Imag}=1 \backslash \backslash$

${ }^{3}$ o-nitrophenyl cation

$\backslash \backslash 1,3 \backslash C, 0,-2.8107432114,2.9931977336,0 . \backslash C, 0,-3.9889228708,2.174045$ $0884,0 . \backslash C, 0,-1.6018084541,2.3590864449,0 . \backslash C, 0,-1.5757919612,0.95706539$ $66,0 . \backslash C, 0,-3.9278300555,0.7471772426,0 . \backslash C, 0,-2.7032899647,0.1133806333$ $, 0 . \backslash \mathrm{H}, 0,-2.8677963145,4.0774267076,0 . \backslash \mathrm{H}, 0,-4.9601249257,2.660551591,0$. $\backslash \mathrm{N}, 0,-0.3180044186,3.087750174,0 . \backslash \mathrm{H}, 0,-4.8504510003,0.1764217995,0 . \backslash \mathrm{H}$, $0,-2.601347693,-0.9668870574,0 . \backslash 0,0,0.6690319506,2.3640785734,0 . \backslash 0,0,-$ $0.3627010807,4.2976556725,0 . \backslash \backslash$ Version=EM64L-G09RevB.01 \State=3-A" \MP2/ GTBas $1=-434.4257793 \backslash \mathrm{MP} 4 / \mathrm{GTBas} 1=-434.526517 \backslash \mathrm{CCSD}(\mathrm{T}) / \mathrm{G} 3 \mathrm{Bas} 1=-434.5210035$ $\backslash \mathrm{MP} 2 / \mathrm{GTBas} 2=-434.4457213 \backslash \mathrm{MP} 4 / \mathrm{GTBas} 2=-434.5480498 \backslash \mathrm{MP} 2 / \mathrm{GTB} a \mathrm{~s} 3=-434.70107$ $53 \backslash \mathrm{MP} 4 / \mathrm{GTBas} 3=-434.8179417 \backslash \mathrm{HF} / \mathrm{GTLargeXP}=-433.3490173 \backslash \mathrm{MP} 2 / \mathrm{GTLargeXP}=-43$ $5.2760576 \backslash \mathrm{HF} / \mathrm{GFHFB} 1=-433.3844432 \backslash \mathrm{HF} / \mathrm{GFHFB} 2=-433.3911653 \backslash \mathrm{G} 4=-435.501384$ $1 \backslash$ FreqCoord $=-5.3115348994,5.6563239782,0 .,-7.5379717911,4.1083498175,0$ $.,-3.0269792956,4.4580273047,0 .,-2.977815249,1.8085914908,0 .,-7.422523$ $1014,1.4119603613,0 .,-5.1084776911,0.2142583456,0 .,-5.4193496393,7.705$ $2198042,0 .,-9.3732776945,5.0277138694,0 .,-0.6009412602,5.8350021956,0$. $,-9.1660240116,0.333388885,0 .,-4.9158347162,-1.8271517399,0 ., 1.2642871$ $607,4.4674610604,0 .,-0.6854057106,8.1213922345,0 . \backslash P G=C S \quad[S G(C 6 H 4 N 102)]$ $\backslash$ NImag=1 \\

${ }^{3}$ p-nitrophenyl cation

$\backslash \backslash 1,3 \backslash C, 0,-2.7249482826,2.8862612846,0 . \backslash C, 0,-3.8921850444,2.140849$ $3301,0 . \backslash C, 0,-1.4418866546,2.1939100327,0 . \backslash C, 0,-1.4700335101,0.82549961$ $11,0 . \backslash C, 0,-3.8817528462,0.7558944398,0 . \backslash C, 0,-2.602391595,0.0567396335$, $0 . \backslash \mathrm{N}, 0,-5.1990932387,2.8505074102,0 . \backslash 0,0,-5.1498930407,4.0660226912,0$. $\backslash \mathrm{O}, 0,-6.1917598945,2.1472800573,0 . \backslash \mathrm{H}, 0,-2.7734363754,3.9708048957,0 . \backslash \mathrm{H}$ $, 0,-0.5213386945,2.7705600901,0 . \backslash \mathrm{H}, 0,-4.8178076681,0.2059939195,0 . \backslash \mathrm{H}, \mathrm{O}$ $,-2.5847831552,-1.0293633956,0 . \backslash \backslash$ Version=EM64L-G0 9RevB.01 \State=3-A" $\backslash \mathrm{M}$ $\mathrm{P} 2 / \mathrm{GTBas} 1=-434.4206799 \backslash \mathrm{MP} 4 / \mathrm{GTBas} 1=-434.5227346 \backslash \mathrm{CCSD}(\mathrm{T}) / \mathrm{G} 3 \mathrm{Bas} 1=-434.516$ $7549 \backslash \mathrm{MP} 2 / \mathrm{GTBas} 2=-434.4406364 \backslash \mathrm{MP} 4 / \mathrm{GTBas} 2=-434.5442526 \backslash \mathrm{MP} 2 / \mathrm{GTB} a \mathrm{~s} 3=-434.6$ $96563 \backslash \mathrm{MP} 4 / \mathrm{GTBas} 3=-434.8148365 \backslash \mathrm{HF} / \mathrm{GTLargeXP}=-433.3417107 \backslash \mathrm{MP} 2 / \mathrm{GTLargeXP}=$ $-435.2717931 \backslash \mathrm{HF} / \mathrm{GFHFB} 1=-433.3770148 \backslash \mathrm{HF} / \mathrm{GFHFB} 2=-433.3837546 \backslash \mathrm{G} 4=-435.500$ $2702 \backslash$ FreqCoord $=-5.1494059803,5.4542433758,0 .,-7.3551637924,4.045618925$ $6,0 .,-2.7247708919,4.1458891219,0 .,-2.7779607403,1.5599681877,0 .,-7.33$ $54497949,1.4284334766,0 \ldots,-4.9178074051,0.1072223681,0 \ldots,-9.8248623605,5$ $.3866783451,0 .,-9.7318874605,7.6836693365,0 .,-11.7007304812,4.05777123$ $89,0 .,-5.2410351964,7.5037337801,0 .,-0.9851873551,5.2355998049,0 .,-9.1$ $043370535,0.3892720928,0 .,-4.8845322763,-1.9452149089,0 . \backslash P G=C S \quad[S G(C 6 H$ $4 \mathrm{~N} 102)] \backslash \mathrm{NImag}=1 \backslash \backslash$ 


\title{
G4MP2 archive entries
}

\begin{abstract}
${ }^{1}$ syn-m-thiomethoxyphenyl cation
$\backslash \backslash 1,1 \backslash \mathrm{C}, 0,-0.2703178765,1.2136560608,-0.1210086609 \backslash \mathrm{C}, 0,-1.5888$

$61379,1.2557567248,-0.1043311574 \backslash \mathrm{C}, 0,-2.6882012185,0.5328315004,0.0393$ $143575 \backslash \mathrm{C}, 0,-2.26519144,-0.8445560275,0.0404742744 \backslash \mathrm{C}, 0,-0.925199557,-1$. $1709869318,0.101029552 \backslash \mathrm{C}, 0,0.0957797321,-0.1886339927,0.0417081524 \backslash \mathrm{H}, 0$ $, 0.4246429814,1.9553717935,-0.4889568963 \backslash \mathrm{H}, 0,-3.6769681248,0.847198004$ $5,0.3464235964 \backslash \mathrm{H}, 0,-3.0345795723,-1.5993295066,0.1728722733 \backslash \mathrm{H}, 0,-0.637$ $313178,-2.210090229,0.2108836937 \backslash \mathrm{S}, 0,1.7283664688,-0.7073783442,-0.181$ $7823457 \backslash \mathrm{C}, 0,2.7235949245,0.7435116882,0.2659764542 \backslash \mathrm{H}, 0,2.422483414,1.1$ $369080333,1.2372681763 \backslash \mathrm{H}, 0,3.7497020853,0.3736027096,0.3185238678 \backslash \mathrm{H}, 0$, $2.67150674,1.5083715169,-0.5131473378 \backslash \backslash V e r s i o n=E M 64 L-G 09 R e v B .01 \backslash$ State= $1-\mathrm{A} \backslash \mathrm{MP} 2 / \mathrm{GTBas} 1=-667.3013051 \backslash \mathrm{CCSD}(\mathrm{T}) / \mathrm{GTBas} 1=-667.4077094 \backslash \mathrm{MP} 2 / \mathrm{GTB} a \mathrm{~s} 2=0 . \backslash$ MP2 / GTBas3=0. \HF / GTMP2LargeXP =-666.4120075 \MP2/GTMP2LargeXP=-667.68064 $85 \backslash \mathrm{HF} / \mathrm{GFHFB} 3=-666.4193412 \backslash \mathrm{HF} / \mathrm{GFHFB} 4=-666.4426194 \backslash \mathrm{G} 4 \mathrm{MP} 2=-667.9000537 \backslash \mathrm{Fr}$ eqCoord $=-0.5108267555,2.2934775744,-0.2286732289,-3.0025128695,2.37303$ $62993,-0.1971573146,-5.079964093,1.0069056107,0.0742933688,-4.28059146$ $02,-1.5959795959,0.076485294,-1.748373781,-2.2128446063,0.1909181847,0$ $.1809974627,-0.3564665855,0.0788169856,0.8024589391,3.6951171777,-0.92$ $39946248,-6.9484627553,1.6009722089,0.6546457231,-5.7345243201,-3.0222$ $947637,0.3266812525,-1.2043473672,-4.1764652618,0.398512427,3.26613928$ $32,-1.3367513429,-0.3435188492,5.1468485042,1.4050334673,0.5026226562$, $4.577830214,2.1484448212,2.3380980062,7.0859100212,0.7060068036,0.6019$ $22877,5.0484161007,2.8504090736,-0.9697079343 \backslash \mathrm{PG}=\mathrm{C} 01 \quad[\mathrm{X}(\mathrm{C} 7 \mathrm{H} 7 \mathrm{~S} 1)] \backslash \mathrm{NImag}$ $=0 \backslash 1$
\end{abstract}

${ }^{1}$ syn-o-thiomethoxyphenyl cation

$\backslash \backslash 1,1 \backslash \mathrm{C}, 0,-0.5046643696,0.8702498652,-0.1190069907 \backslash \mathrm{C}, 0,-1.6913$

$450434,1.4577284423,-0.0203677981 \backslash \mathrm{C}, 0,-2.635205575,0.3928844641,0.0722$ $090241 \backslash \mathrm{C}, 0,-2.1928697702,-0.932060011,0.0820186809 \backslash \mathrm{C}, 0,-0.8481636323,-$ $1.2503737376,0.0052842893 \backslash \mathrm{C}, 0,0.2059303427,-0.2516850762,-0.0979212354$ $\backslash \mathrm{H}, 0,-1.960203219,2.4980556142,-0.1434359115 \backslash \mathrm{H}, 0,-3.6851709998,0.66541$ $86011,0.0461059954 \backslash \mathrm{H}, 0,-2.91521536,-1.7355025068,0.1667183277 \backslash \mathrm{H}, 0,-0.4$ $866197916,-2.270221675,0.1203428656 \backslash \mathrm{S}, 0,1.8936285689,-0.6545618333,-0$. $0639806631 \backslash \mathrm{C}, 0,2.6657997891,0.9870049051,0.134359282 \backslash \mathrm{H}, 0,2.4304522603$, $1.4134592967,1.1095708741 \backslash \mathrm{H}, 0,3.7385493712,0.7943510389,0.0735309583 \backslash \mathrm{H}$ $, 0,2.3742004288,1.6496466122,-0.6833936985 \backslash \backslash$ Version=EM64L-G09RevB.01\S tate $=1-\mathrm{A} \backslash \mathrm{MP} 2 / \mathrm{GTBas} 1=-667.2949862 \backslash \mathrm{CCSD}(\mathrm{T}) / \mathrm{GTBas} 1=-667.3993963 \backslash \mathrm{MP} 2 / \mathrm{GTB} a \mathrm{~s}$ 2=0. $\backslash \mathrm{MP} 2 / \mathrm{GTBas} 3=0 . \backslash \mathrm{HF} / \mathrm{GTMP} 2 \mathrm{LargeXP}=-666.4152106 \backslash \mathrm{MP} 2 / \mathrm{GTMP} 2 \mathrm{LargeXP}=-667$. $6737821 \backslash \mathrm{HF} / \mathrm{GFHFB} 3=-666.4227138 \backslash \mathrm{HF} / \mathrm{GFHFB} 4=-666.4459593 \backslash \mathrm{G} 4 \mathrm{MP} 2=-667.89230$ $26 \backslash$ FreqCoord=-0.9536774475, $1.6445339124,-0.2248906202,-3.1961789283,2$. $7547075321,-0.0384895604,-4.9798168406,0.742444039,0.1364552798,-4.143$ $9233107,-1.7613381602,0.1549928446,-1.6027969809,-2.3628639278,0.00998$ $58597,0.3891519501,-0.4756158658,-0.1850443175,-3.7042472488,4.7206409$ $756,-0.2710545903,-6.9639639425,1.2574589198,0.0871277044,-5.508958648$ $8,-3.2796244407,0.3150519807,-0.919578137,-4.2900972266,0.227415058,3$. $5784393926,-1.236942602,-0.1209059311,5.0376315265,1.8651689624,0.2539$ $022464,4.592889151,2.6710509708,2.096785077,7.0648344459,1.501105917,0$ $.1389533735,4.4865885949,3.1173803131,-1.2914269312 \backslash \mathrm{PG}=\mathrm{C} 01 \quad[\mathrm{X}(\mathrm{C} 7 \mathrm{H} 7 \mathrm{~S} 1)]$ $\backslash$ NImag $=0 \backslash \backslash$

${ }^{1}$ syn-m-methoxyphenyl cation

$\backslash \backslash 1,1 \backslash \mathrm{C}, 0,1.2388739653,-1.2117427224,-0.0186943332 \backslash \mathrm{C}, 0,-0.0802$

$180973,-1.1532263875,-0.0491054784 \backslash \mathrm{C}, 0,-0.4409603319,0.2550351087,0.01$ $41059292 \backslash \mathrm{C}, 0,0.595700528,1.2227442485,-0.0078035276 \backslash \mathrm{C}, 0,1.9197408959,0$ $.8521822126,-0.0238216376 \backslash \mathrm{C}, 0,2.3628214662,-0.5317958561,0.0405718955 \backslash$ 
$\mathrm{H}, 0,-0.773610901,-1.9032537786,-0.4076041595 \backslash \mathrm{H}, 0,0.3153639999,2.268429$ $2668,0.0102839579 \backslash \mathrm{H}, 0,2.706069896,1.592573585,0.0977744835 \backslash \mathrm{H}, 0,3.35694$ $7686,-0.8184835805,0.3514997502 \backslash 0,0,-1.6794094484,0.6568228058,-0.1123$ $334112 \backslash \mathrm{C}, 0,-2.7811768272,-0.2445333921,0.1218362959 \backslash \mathrm{H}, 0,-2.624652815,-$ $0.8029074681,1.0481653976 \backslash \mathrm{H}, 0,-3.6607699515,0.3916819795,0.2015736355 \backslash$ $\mathrm{H}, 0,-2.8934090649,-0.9191500217,-0.7328717977 \backslash \backslash$ Version=EM64L-G0 9RevB. 0 $1 \backslash$ State $=1-A \backslash M P 2 / G T B a s 1=-344.6906472 \backslash \mathrm{CCSD}(\mathrm{T}) / \mathrm{GTBas} 1=-344.7856435 \backslash \mathrm{MP} 2 / \mathrm{GT}$ Bas2=0. \MP2 / GTBas3=0. \HF / GTMP2LargeXP=-343.7514905 \MP2/GTMP2 LargeXP=-3 $45.0686051 \backslash \mathrm{HF} / \mathrm{GFHFB}=-343.7548103 \backslash \mathrm{HF} / \mathrm{GFHFB} 4=-343.7798429 \backslash \mathrm{G} 4 \mathrm{MP} 2=-345.27$ $118 \backslash$ FreqCoord=2.3411325075, -2.2898618888,-0.03532717, -0.1515902349, -2. $1792820416,-0.0927959058,-0.8332942627,0.4819465097,0.026656343,1.1257$ $108551,2.3106517603,-0.01474653,3.6277845394,1.6103909971,-0.045016371$ $, 4.465085472,-1.0049485267,0.0766697711,-1.4619127364,-3.5966284029,-0$ $.770260232,0.595951592,4.286710066,0.0194338639,5.1137309998,3.0095279$ $222,0.1847669966,6.3437117689,-1.5467098114,0.6642382636,-3.1736239225$ $, 1.2412152208,-0.2122793827,-5.2556625306,-0.4621011414,0.2302372323,-$ $4.9598750143,-1.5172752247,1.9807455434,-6.9178526438,0.7401716724,0.3$ $809189666,-5.467750723,-1.7369418161,-1.3849269882 \backslash \mathrm{PG}=\mathrm{C} 01 \quad[\mathrm{X}(\mathrm{C} 7 \mathrm{H} 7 \mathrm{O} 1)] \backslash$ NImag $=0 \backslash \backslash$

${ }^{1}$ syn-o-methoxyphenyl cation

$\backslash \backslash 1,1 \backslash \mathrm{C}, 0,-1.3496507949,1.4112771453,0.0158660867 \backslash \mathrm{C}, 0,-0.13336$

$41303,0.8842959891,-0.0588592095 \backslash \mathrm{C}, 0,0.5700651976,-0.261467636,-0.1445$ $408891 \backslash \mathrm{C}, 0,-0.4845836408,-1.2462334032,-0.0368105578 \backslash \mathrm{C}, 0,-1.8382120363$ $,-0.9590201907,0.0576979656 \backslash \mathrm{C}, 0,-2.3029498504,0.3527041968,0.106228190$ $6 \backslash \mathrm{H}, 0,-1.6303088888,2.4380330795,-0.1897675663 \backslash \mathrm{H}, 0,-0.0847238165,-2.24$ $07260603,0.1628763873 \backslash \mathrm{H}, 0,-2.5397257154,-1.7828125018,0.1247611747 \backslash \mathrm{H}, 0$ $,-3.3541598742,0.6153535667,0.0640920245 \backslash 0,0,1.829624787,-0.5904390136$ $,-0.1365322415 \backslash \mathrm{C}, 0,2.7808143738,0.4641899067,0.1642861894 \backslash \mathrm{H}, 0,2.716281$ $1628,0.7266444326,1.2225469017 \backslash \mathrm{H}, 0,3.7596273275,0.051896703,-0.0698169$ $636 \backslash \mathrm{H}, 0,2.5695178991,1.332750786,-0.4711854926 \backslash \backslash$ Version=EM64L-G0 9RevB. $01 \backslash$ State $=1-A \backslash M P 2 / G T B a s 1=-344.6747495 \backslash \mathrm{CCSD}(\mathrm{T}) / \mathrm{GTBas} 1=-344.7707148 \backslash \mathrm{MP} 2 / \mathrm{G}$ TBas2=0 . \MP2 / GTBas3=0 . \HF/GTMP2LargeXP=-343.7400124 \MP2/GTMP2LargeXP=$345.0532775 \backslash \mathrm{HF} / \mathrm{GFHFB} 3=-343.743477 \backslash \mathrm{HF} / \mathrm{GFHFB} 4=-343.7684919 \backslash \mathrm{G} 4 \mathrm{MP} 2=-345.25$ $77801 \backslash$ FreqCoord=-2.5504703774,2.6669273022,0.0299825587,-0.2520216822, $1.6710772397,-0.1112277864,1.0772671014,-0.4941022246,-0.2731426953,-0$ $.9157303696,-2.3550398296,-0.0695618731,-3.4737173229,-1.8122855164,0$. $1090333533,-4.351944515,0.6665143378,0.2007421878,-3.0808373119,4.6072$ $148232,-0.3586087292,-0.1601048101,-4.2343585929,0.3077917655,-4.79938$ $60548,-3.3690273746,0.2357644522,-6.3384435681,1.1628497159,0.12111637$ $35,3.4574897733,-1.1157680339,-0.2580085447,5.2549775929,0.8771917972$, $0.3104559054,5.1330274975,1.3731589737,2.3102788287,7.1046660106,0.098$ $0705559,-0.1319349407,4.8556851229,2.5185339889,-0.8904115387 \backslash P G=C 01 \quad[$ $\mathrm{X}(\mathrm{C} 7 \mathrm{H} 7 \mathrm{O} 1)] \backslash \mathrm{NImag}=0 \backslash \backslash$

${ }^{1}$ anti-m-thiomethoxyphenyl cation

$\backslash \backslash 1,1 \backslash \mathrm{C}, 0,-3.5279323536,3.6141388216,0.2165152389 \backslash \mathrm{C}, 0,-4.7124$

$064702,2.9557743923,-0.0571486728 \backslash \mathrm{C}, 0,-2.2883730699,2.9380702829,0.284$ $8509056 \backslash \mathrm{C}, 0,-4.7645669376,1.520985275,-0.1785143756 \backslash \mathrm{C}, 0,-3.5058041501$, $1.114494167,-0.1455418325 \backslash \mathrm{C}, 0,-2.2547026348,1.499913423,0.0177416266 \backslash \mathrm{H}$ $, 0,-5.67509856,0.9530684105,-0.0421286856 \backslash \mathrm{S}, 0,-0.7337306188,3.68040808$ $73,0.4273862788 \backslash \mathrm{H}, 0,-3.559542569,4.6794208161,0.4042375829 \backslash \mathrm{H}, 0,-5.6654$ $854897,3.4740748945,-0.0016570766 \backslash \mathrm{C}, 0,-0.9883244209,5.4054459521,-0.09$ $72141396 \backslash \mathrm{H}, 0,0.0167699175,5.830260934,-0.134593513 \backslash \mathrm{H}, 0,-1.58430279,5.9$ $531441692,0.6337321722 \backslash \mathrm{H}, 0,-1.4362730908,5.4407218415,-1.0910013909 \backslash \mathrm{H}$, $0,-1.3461567621,0.9851185332,-0.2619141186 \backslash \backslash$ Version=EM64L-G09RevB.01\S tate $=1-\mathrm{A} \backslash \mathrm{MP} 2 / \mathrm{GTBas} 1=-667.2995536 \backslash \mathrm{CCSD}(\mathrm{T}) / \mathrm{GTBas} 1=-667.4057641 \backslash \mathrm{MP} 2 / \mathrm{GTB}$ S 
2=0. $\backslash$ MP2 $/$ GTBas3=0. $\backslash \mathrm{HF} /$ GTMP2LargeXP $=-666.4116043 \backslash \mathrm{MP} 2 /$ GTMP2 LargeXP $=-667$. $678911 \backslash \mathrm{HF} / \mathrm{GFHFB} 3=-666.4189761 \backslash \mathrm{HF} / \mathrm{GFHFB} 4=-666.442232 \backslash \mathrm{G} 4 \mathrm{MP} 2=-667.8981692$ $\backslash$ FreqCoord $=-6.6668259636,6.829732579,0.4091545052,-8.9051576556,5.5856$ $041121,-0.1079953405,-4.3243983919,5.5521481939,0.5382902003,-9.003726$ $654,2.8742456218,-0.3373432807,-6.6250097192,2.1060887523,-0.275034204$ $3,-4.2607704908,2.8344255926,0.0335268155,-10.7243820556,1.8010382817$, $-0.0796116781,-1.3865499249,6.9549633422,0.8076430199,-6.7265606137,8$. $8428238029,0.7638983244,-10.7062159854,6.5650501158,-0.0031314209,-1.8$ $676624859,10.2148124755,-0.1837081001,0.0316905513,11.0175964484,-0.25$ $43448788,-2.9938983847,11.2498121094,1.1975802471,-2.7141627936,10.281$ $4742456,-2.0616938393,-2.5438676122,1.8616042361,-0.4949459545 \backslash P G=C 01$ $[\mathrm{X}(\mathrm{C} 7 \mathrm{H} 7 \mathrm{~S} 1)] \backslash \mathrm{NImag}=0 \backslash \backslash$

'anti-o-thiomethoxyphenyl cation

$\backslash \backslash 1,1 \backslash C, 0,-3.4992199216,3.6064392283,-0.0008174707 \backslash C, 0,-4.707$

$624665,2.9242740382,-0.0004417656 \backslash C, 0,-2.1961459093,2.9596243628,-0.00$ $19297461 \backslash \mathrm{C}, 0,-4.7631747504,1.5316445136,-0.0010966216 \backslash \mathrm{C}, 0,-3.548373326$ $4,0.7846960274,-0.0021315737 \backslash \mathrm{C}, 0,-2.5788397035,1.6882666641,-0.0015031$ $015 \backslash \mathrm{H}, 0,-5.6908457268,0.9698934717,0.000660632 \backslash \mathrm{S}, 0,-0.6088696546,3.679$ $9300313,-0.0057279607 \backslash \mathrm{H}, 0,-3.4591316596,4.6917418727,-0.002202989 \backslash \mathrm{H}, 0$, $-5.6268254876,3.498071948,0.0000013614 \backslash C, 0,-0.9850207681,5.4586448015$, $-0.0031566873 \backslash \mathrm{H}, 0,0.0019160501,5.9275887237,-0.0043373618 \backslash \mathrm{H}, 0,-1.51522$ $68666,5.7513035735,0.9040770115 \backslash \mathrm{H}, 0,-1.5182784137,5.7532155829,-0.9079$ $929082 \backslash \mathrm{H}, 0,-3.486059197,-0.2949748398,-0.0009408187 \backslash \backslash$ Version=EM64L-G09 RevB.01 \State=1-A \MP2/GTBas1=-667.2951367 \CCSD $(T) / G T B a s 1=-667.3992662 \backslash$ MP2 / GTBas2=0. \MP2/GTBas3=0. \HF/GTMP2LargeXP=-666.4158365 MMP2/GTMP2Larg $\mathrm{eXP}=-667.6743586 \backslash \mathrm{HF} / \mathrm{GFHFB} 3=-666.4233763 \backslash \mathrm{HF} / \mathrm{GFHFB} 4=-666.4466258 \backslash \mathrm{G} 4 \mathrm{MP} 2=-$ $667.8925204 \backslash$ FreqCoord $=-6.6125673305,6.8151824564,-0.0015447957,-8.8961$ $213532,5.5260770697,-0.0008348159,-4.1501143165,5.5928795019,-0.003646$ $6916,-9.0010958013,2.8943886637,-0.0020723145,-6.7054538042,1.48286058$ $94,-0.0040280905,-4.8733007801,3.1903616344,-0.0028404502,-10.75413988$ $82,1.8328330397,0.0012484136,-1.1505968978,6.9540599473,-0.010824277,-$ $6.5368114942,8.8661072257,-0.0041630459,-10.633159169,6.6103979748,0.0$ $000025726,-1.8614194869,10.3153437316,-0.0059652745,0.0036208099,11.20$ $15193162,-0.0081964259,-2.8633638071,10.8683886609,1.7084579547,-2.869$ $1303953,10.8720018352,-1.7158579272,-6.5876971654,-0.5574216633,-0.001$ $7778897 \backslash \mathrm{PG}=\mathrm{C} 01 \quad[\mathrm{X}(\mathrm{C} 7 \mathrm{H} 7 \mathrm{~S} 1)] \backslash \mathrm{NImag}=0 \backslash \backslash$

${ }^{1}$ anti-m-methoxyphenyl cation

$\backslash \backslash 1,1 \backslash C, 0,-3.5424455642,3.7492821724,0.0709901292 \backslash C, 0,-4.6737$

$61527,2.9757496893,-0.100059086 \backslash C, 0,-2.2625617554,3.1572779772,0.19309$ $02871 \backslash \mathrm{C}, 0,-4.6704555401,1.5271561672,-0.1310249394 \backslash \mathrm{C}, 0,-3.3875755101,1$ $.2369637075,-0.0465195089 \backslash C, 0,-2.1622570246,1.7099590077,0.0561433617 \backslash$ $\mathrm{H}, 0,-5.5528959273,0.9338837761,0.0591578958 \backslash 0,0,-1.1138025783,3.777489$ $4161,0.2573565166 \backslash \mathrm{H}, 0,-3.6484967782,4.8227108194,0.1524508208 \backslash \mathrm{H}, 0,-5.6$ $613268193,3.4240072264,-0.0222780266 \backslash C, 0,-1.0290590854,5.1961635202,-0$ $.0286440493 \backslash \mathrm{H}, 0,0.036059255,5.4166935186,-0.0494604496 \backslash \mathrm{H}, 0,-1.51524561$ $43,5.7619660885,0.7698686117 \backslash \mathrm{H}, 0,-1.4846724729,5.4114044941,-0.9981056$ $099 \backslash \mathrm{H}, 0,-1.2208730578,1.2457124193,-0.2082359532 \backslash \backslash$ Version=EM64L-G0 9Rev B.01 \State $=1-A \backslash M P 2 / G T B a s 1=-344.6903398 \backslash \mathrm{CCSD}(\mathrm{T}) / \mathrm{GTBas} 1=-344.7849274 \backslash \mathrm{MP} 2$ /GTBas2=0. \MP2/GTBas3=0 . \HF/GTMP2LargeXP=-343.7518832\MP2 / GTMP2LargeXP $=-345.0683321 \backslash \mathrm{HF} / \mathrm{GFHFB} 3=-343.7552177 \backslash \mathrm{HF} / \mathrm{GFHFB} 4=-343.7802361 \backslash \mathrm{G} 4 \mathrm{MP} 2=-345$ $.2703935 \backslash$ FreqCoord $=-6.694251957,7.0851165007,0.1341519024,-8.832129296$ $5,5.6233519528,-0.1890842696,-4.2756220765,5.9663907023,0.3648877616,-$ $8.8258818866,2.8859069182,-0.2476012521,-6.4015899686,2.3375226434,-0$. $0879091316,-4.0860736054,3.231354223,0.1060955778,-10.4934525469,1.764$ $7845769,0.1117922216,-2.1047818391,7.1384204663,0.4863333348,-6.894659$ 
$7076,9.1136026668,0.2880903001,-10.6983572373,6.470435935,-0.042099369$ $,-1.9446398459,9.8193259948,-0.0541294085,0.0681421166,10.2360672959,-$ $0.0934667042,-2.8633992351,10.8885378942,1.4548408344,-2.8056243707,10$ $.2260724881,-1.8861462544,-2.3071157223,2.3540553128,-0.3935089226 \backslash P G=$ $\mathrm{C} 01[\mathrm{X}(\mathrm{C} 7 \mathrm{H} 7 \mathrm{O} 1)] \backslash \mathrm{NImag}=0 \backslash \backslash$

'anti-o-methoxyphenyl cation

$\backslash \backslash 1,1 \backslash \mathrm{C}, 0,-3.5293817276,3.7140423176,-0.0034641923 \backslash \mathrm{C}, 0,-4.691$

$4133754,2.9530086807,-0.0023388108 \backslash \mathrm{C}, 0,-2.192245919,3.1725231001,-0.00$ $1854027 \backslash \mathrm{C}, 0,-4.6553987639,1.5623079674,0.0010076461 \backslash \mathrm{C}, 0,-3.3897090875$, $0.9048733613,0.0031672846 \backslash \mathrm{C}, 0,-2.4656724508,1.8529015151,0.0013049187 \backslash$ $\mathrm{H}, \mathrm{0},-5.5400004353,0.9354731291,-0.0002118721 \backslash \mathrm{H}, 0,-3.2578485097,-0.1697$ $823395,0.0022908778 \backslash 0,0,-1.0404354545,3.7919322964,-0.0002991954 \backslash \mathrm{H}, 0,-$ $3.560562366,4.8014728418,-0.0022852247 \backslash \mathrm{H}, 0,-5.6463730642,3.4650761188$, $-0.0037208943 \backslash \mathrm{C}, 0,-1.0118176217,5.240040026,-0.0044492364 \backslash \mathrm{H}, 0,0.047076$ $3254,5.4905322595,-0.0040993006 \backslash \mathrm{H}, 0,-1.4911265096,5.6297557601,0.89714$ $26807 \backslash \mathrm{H}, 0,-1.4894510401,5.6245629657,-0.9091406544 \backslash \backslash$ Version=EM64L-G09R evB.01 \State=1-A \MP2/GTBas1=-344.6721077\CCSD (T) / GTBas $1=-344.7676939 \backslash \mathrm{M}$ P2 / GTBas2=0 . \MP2/GTBas3=0 . \HF/GTMP2LargeXP=-343. 7431715 \MP2/GTMP2Large $\mathrm{XP}=-345.051469 \backslash \mathrm{HF} / \mathrm{GFHFB} 3=-343.7467435 \backslash \mathrm{HF} / \mathrm{GFHFB} 4=-343.7717539 \backslash \mathrm{G} 4 \mathrm{MP} 2=-34$ $5.2562328 \backslash$ FreqCoord $=-6.6695648835,7.0185228261,-0.0065463747,-8.865486$ $4557,5.5803776745,-0.0044197119,-4.1427444029,5.9951998093,-0.00350360$ $34,-8.7974287031,2.9523341935,0.0019041752,-6.4056218456,1.7099628378$, $0.0059853005,-4.6594456654,3.5014764148,0.002465939,-10.4690835988,1.7$ $677880186,-0.0004003802,-6.1564414658,-0.3208421238,0.0043291317,-1.96$ $61380679,7.1657135547,-0.0005653974,-6.7284877508,9.0734687054,-0.0043$ $184488,-10.6700987355,6.5480448942,-0.0070314712,-1.9120582015,9.90224$ $05744,-0.0084078382,0.0889613623,10.3756022942,-0.0077465556,-2.817820$ $7326,10.6386965817,1.6953539687,-2.8146545541,10.6288836224,-1.7180268$ $531 \backslash \mathrm{PG}=\mathrm{C} 01 \quad[\mathrm{X}(\mathrm{C} 7 \mathrm{H} 7 \mathrm{O} 1)] \backslash \mathrm{NImag}=1 \backslash \backslash$

${ }^{3}$ syn-m-thiomethoxyphenyl cation

$\backslash \backslash 1,3 \backslash \mathrm{C}, 0,0.3075302504,1.2114394396,0.0000221904 \backslash \mathrm{C}, 0,1.6467$

$976482,1.4270000391,0.0001038764 \backslash C, 0,2.6510366872,0.4644922094,0.00011$ $39566 \backslash \mathrm{C}, 0,2.2477750728,-0.8846594831,0.0000048338 \backslash \mathrm{C}, 0,0.9020671516,-1$. $194450014,-0.0000864373 \backslash \mathrm{C}, 0,-0.087046425,-0.1581285315,-0.0000762197 \backslash \mathrm{H}$ $, 0,-0.4138930038,2.0184436445,-0.000016554 \backslash \mathrm{H}, 0,3.7017488917,0.73542504$ $15,0.0001930725 \backslash \mathrm{H}, 0,2.9904122817,-1.6744383463,-0.0000037942 \backslash \mathrm{H}, 0,0.579$ $3404108,-2.2302336047,-0.0001746545 \backslash S, 0,-1.7049756354,-0.7206126683,-0$ $.0002299349 \backslash \mathrm{C}, 0,-2.7661826184,0.7461483559,0.000108013 \backslash \mathrm{H}, 0,-2.59316972$ $38,1.3370944828,-0.901645403 \backslash \mathrm{H}, 0,-3.7862647723,0.3586019594,-0.0008349$ $811 \backslash \mathrm{H}, 0,-2.5943882159,1.3358674757,0.9028910207 \backslash \backslash$ Version=EM6 4L-G09RevB $.01 \backslash \mathrm{State}=3-\mathrm{A} \backslash \mathrm{MP} 2 / \mathrm{GTBas} 1=-667.2873088 \backslash \mathrm{CCSD}(\mathrm{T}) / \mathrm{GTBas} 1=-667.4073003 \backslash \mathrm{MP} 2 /$ GTBas2=0. \MP2/GTBas3=0. \HF / GTMP2LargeXP=-666.4549376 \MP2/GTMP2LargeXP= $-667.6595742 \backslash \mathrm{HF} / \mathrm{GFHFB} 3=-666.4619855 \backslash \mathrm{HF} / \mathrm{GFHFB} 4=-666.4853947 \backslash \mathrm{G} 4 \mathrm{MP} 2=-667$. $8935574 \backslash$ FreqCoord=0 .5811479509,2.2892887674,0.0000419337,3.1119965514, $2.6966392655,0.0001962979,5.009733307,0.8777630665,0.0002153468,4.2476$ $792959,-1.6717641439,0.0000091346,1.7046598701,-2.257183406,-0.0001633$ $429,-0.1644939041,-0.2988196183,-0.0001440344,-0.7821444255,3.81430570$ $28,-0.0000312825,6.995291618,1.3897519197,0.0003648542,5.6510602369,-3$ $.1642299009,-0.00000717,1.094794714,-4.2145307253,-0.0003300493,-3.221$ $9370141,-1.361760591,-0.0004345139,-5.2273275823,1.4100160472,0.000204$ $115,-4.900380594,2.5267423863,-1.7038628806,-7.1550034862,0.677659494$, $-0.0015778857,-4.9026832104,2.524423679,1.706216757 \backslash \mathrm{PG}=\mathrm{C} 01 \quad[\mathrm{X}(\mathrm{C} 7 \mathrm{H} 7 \mathrm{~S} 1)]$ $\backslash N I m a g=0 \backslash \backslash$ 


\begin{abstract}
${ }^{3}$ syn-o-thiomethoxyphenyl cation
$\backslash \backslash 1,3 \backslash \mathrm{C}, 0,0.8345047317,-1.2864220919,0.0000123339 \backslash \mathrm{C}, 0,-0.14$ $4356751,-0.238942352,0.0000671438 \backslash C, 0,0.3301072595,1.0785482291,0.0001$ $348146 \backslash C, 0,1.6372819608,1.4508807519,0.0001502544 \backslash C, 0,2.5802651046,0.3$ $97883163,0.0000992712 \backslash \mathrm{C}, 0,2.1690533194,-0.9524126298,0.0000285945 \backslash \mathrm{H}, 0$, $0.511132607,-2.3223986486,-0.0000437328 \backslash \mathrm{H}, 0,1.9551470203,2.4874514809$, $0.000197374 \backslash \mathrm{H}, 0,3.6391504199,0.6364869313,0.0001089865 \backslash \mathrm{H}, 0,2.918506533$ $1,-1.7351145894,-0.0000141067 \backslash \mathrm{S}, 0,-1.7944293416,-0.673330563,0.0000295$ $561 \backslash \mathrm{C}, 0,-2.6918101192,0.9025379238,0.0001495506 \backslash \mathrm{H}, 0,-2.440833381,1.467$ $0880997,-0.8995160431 \backslash \mathrm{H}, 0,-3.7495033318,0.6355699826,-0.0000829776 \backslash \mathrm{H}, \mathrm{O}$ $,-2.4411610317,1.4667143127,0.9001469807 \backslash \backslash$ Version=EM64L-G0 9RevB.01 \Sta te $=3-\mathrm{A} \backslash \mathrm{MP} 2 / \mathrm{GTBas} 1=-667.276298 \backslash \mathrm{CCSD}(\mathrm{T}) / \mathrm{GTBas} 1=-667.4108577 \backslash \mathrm{MP} 2 / \mathrm{GTBas} 2=0$ . \MP2 / GTBas3=0. \HF/GTMP2LargeXP =-666.471068\MP2/GTMP2LargeXP=-667.6471 $08 \backslash \mathrm{HF} / \mathrm{GFHFB} 3=-666.4781456 \backslash \mathrm{HF} / \mathrm{GFHFB} 4=-666.5015256 \backslash \mathrm{G} 4 \mathrm{MP} 2=-667.8958017 \backslash \mathrm{Fr}$ eqCoord $=1.5769853995,-2.430985445,0.0000233077,-0.2727947249,-0.451535$ $6069,0.0001268835,0.6238123149,2.0381607741,0.0002547626,3.0940145082$, $2.7417672725,0.0002839396,4.875994398,0.7518902109,0.0001875953,4.0989$ $167413,-1.7997990359,0.0000540358,0.9659006449,-4.3886974173,-0.000082$ $643,3.6946924179,4.7006020678,0.0003729828,6.87699765,1.2027859873,0.0$ $002059547,5.5151780646,-3.278891383,-0.0000266579,-3.3909800204,-1.272$ $410361,0.0000558529,-5.086783927,1.7055495004,0.0002826096,-4.61250662$ $62,2.7723947212,-1.6998389737,-7.0855344314,1.2010532054,-0.000156805$, $-4.6131257962,2.7716883661,1.7010312729 \backslash \mathrm{PG}=\mathrm{C} 01 \quad[\mathrm{X}(\mathrm{C} 7 \mathrm{H} 7 \mathrm{~S} 1)] \backslash \mathrm{NImag}=0 \backslash \backslash$
\end{abstract}

${ }^{3}$ syn-m-methoxyphenyl cation

$\backslash \backslash 1,3 \backslash C, 0,-1.2704218276,1.4026830375,0.0000255209 \backslash \mathrm{C}, 0,0.0599$

$309301,1.1720673711,0.0000249552 \backslash \mathrm{C}, 0,0.4238038553,-0.2128437795,-0.000$ $0027432 \backslash C, 0,-0.5929251056,-1.2414738177,-0.0000208332 \backslash C, 0,-1.926874839$ $8,-0.9103458421,-0.0000218542 \backslash \mathrm{C}, 0,-2.3027417038,0.4508867037,0.0000070$ $044 \backslash \mathrm{H}, 0,0.8010368747,1.9615609077,0.0000520437 \backslash \mathrm{H}, 0,-0.2529041908,-2.27$ $05527983,-0.0000400679 \backslash \mathrm{H}, 0,-2.6871476092,-1.6830024939,-0.0000427722 \backslash \mathrm{H}$ $, 0,-3.3464724642,0.7476504653,-0.0000000214 \backslash 0,0,1.6445206657,-0.664249$ $7762,-0.0000099716 \backslash \mathrm{C}, 0,2.797942928,0.2203226727,-0.0000414702 \backslash \mathrm{H}, 0,2.78$ $4235361,0.8354678446,0.9022524242 \backslash \mathrm{H}, 0,3.6588918218,-0.4438924346,-0.00$ $02059901 \backslash \mathrm{H}, 0,2.7840503043,0.8356799398,-0.9021862244 \backslash \backslash$ Version=EM64L-G0 9RevB.01 \State=3-A \MP2/GTBas $1=-344.6686239 \backslash \mathrm{CCSD}(\mathrm{T}) / \mathrm{GTBas} 1=-344.778605 \backslash$ MP2 / GTBas2=0 . \MP2 / GTBas3=0 . \HF/GTMP2LargeXP=-343. $7892523 \backslash$ MP2 / GTMP2Larg $\mathrm{eXP}=-345.0397551 \backslash \mathrm{HF} / \mathrm{GFHFB} 3=-343.7924452 \backslash \mathrm{HF} / \mathrm{GFHFB} 4=-343.8174548 \backslash \mathrm{G} 4 \mathrm{MP} 2=-$ $345.2583627 \backslash$ FreqCoord $=-2.4007493274,2.650686792,0.0000482275,0.1132530$ $448,2.2148863407,0.0000471585,0.8008732206,-0.4022164523,-0.000005184$, $-1.120466067,-2.3460455167,-0.000039369,-3.6412657395,-1.7203043277,-0$ $.0000412985,-4.3515511749,0.8520523869,0.0000132365,1.5137403156,3.706$ $8129085,0.0000983483,-0.4779196584,-4.290722959,-0.0000757174,-5.07797$ $30601,-3.1804137944,-0.0000808278,-6.3239164685,1.4128546225,-0.000000$ $0404,3.1076936781,-1.2552501609,-0.0000188436,5.2873458693,0.416349512$ $2,-0.0000783673,5.2614423219,1.5788054191,1.7050099845,6.914303493,-0$. $8388351339,-0.0003892649,5.2610926152,1.5792062209,-1.704884885 \backslash \mathrm{PG}=\mathrm{C} 01$ $[\mathrm{X}(\mathrm{C} 7 \mathrm{H} 7 \mathrm{O} 1)] \backslash \mathrm{NImag}=0 \backslash \backslash$

${ }^{3}$ syn-o-methoxyphenyl cation

$\backslash \backslash 1,3 \backslash \mathrm{C}, 0,-1.2888139833,1.4275274467,-0.0006786727 \backslash \mathrm{C}, 0,0.014$

$8128486,1.0672088517,-0.0012472669 \backslash \mathrm{C}, 0,0.4883194497,-0.27119726,-0.000$ $8103408 \backslash \mathrm{C}, 0,-0.4941928464,-1.3242836077,0.0003625948 \backslash \mathrm{C}, 0,-1.8215014662$ $,-0.9919420537,0.000964146 \backslash \mathrm{C}, 0,-2.2359320288,0.367626256,0.0005215453 \backslash$ $\mathrm{H}, 0,-1.6139010831,2.4624731529,-0.0004620114 \backslash \mathrm{H}, 0,-0.140613594,-2.34949$ $65387,0.0004437735 \backslash \mathrm{H}, 0,-2.5748882408,-1.7710246897,0.00169012 \backslash \mathrm{H}, 0,-3.2$ $950026002,0.6056262458,0.0014821169 \backslash 0,0,1.73601705,-0.6150007574,-0.00$ 
$18342466 \backslash \mathrm{C}, 0,2.7846205378,0.3997366476,0.0020718807 \backslash \mathrm{H}, 0,2.7326534484,0$ $.9560794419,0.9401392763 \backslash \mathrm{H}, 0,3.7148625762,-0.1581821155,-0.0717709384 \backslash$ $\mathrm{H}, 0,2.6489839322,1.0601719799,-0.8564139768 \backslash \backslash$ Version=EM64L-G09RevB .01 State $=3-\mathrm{A} \backslash \mathrm{MP} 2 / \mathrm{GTBas} 1=-344.6602029 \backslash \mathrm{CCSD}(\mathrm{T}) / \mathrm{GTBas} 1=-344.7804869 \backslash \mathrm{MP} 2 / \mathrm{GTBa}$ $\mathrm{s} 2=0 . \backslash \mathrm{MP} 2 / \mathrm{GTBas} 3=0 . \backslash \mathrm{HF} / \mathrm{GTMP} 2 \mathrm{LargeXP}=-343.7988845 \backslash \mathrm{MP} 2 / \mathrm{GTMP} 2 \mathrm{LargeXP}=-345$ $.0296432 \backslash \mathrm{HF} / \mathrm{GFHFB} 3=-343.802122 \backslash \mathrm{HF} / \mathrm{GFHFB} 4=-343.8271326 \backslash \mathrm{G} 4 \mathrm{MP} 2=-345.25883$ $37 \backslash$ FreqCoord $=-2.4355054647,2.6976359214,-0.0012825055,0.0279922271,2.0$ $167324563,-0.0023569929,0.9227900253,-0.5124885494,-0.0015313222,-0.93$ $38891365,-2.5025333408,0.0006852049,-3.4421389218,-1.8744988212,0.0018$ $219719,-4.2252991862,0.6947129431,0.0009855778,-3.0498310526,4.6533998$ $686,-0.000873075,-0.2657211832,-4.4399050083,0.0008386104,-4.865833597$ $9,-3.3467516381,0.0031938639,-6.2266525215,1.1444677434,0.002800795,3$. $2805967865,-1.162183003,-0.0034662237,5.2621702005,0.7553927892,0.0039$ $152871,5.1639666336,1.8067283065,1.776605759,7.0200728903,-0.298920877$ $4,-0.1356274179,5.0058541623,2.0034346958,-1.6183878725 \backslash \mathrm{PG}=\mathrm{C} 01 \quad[\mathrm{X}(\mathrm{C} 7 \mathrm{H} 7$ 01) ] \NImag=1 \\

3anti-m-thiomethoxyphenyl cation

$\backslash \backslash 1,3 \backslash \mathrm{C}, 0,-3.5245933647,3.6377126389,-0.0029846047 \backslash \mathrm{C}, 0,-4.7$

$223158176,2.9543900364,-0.0025010073 \backslash \mathrm{C}, 0,-2.2926162422,2.9245455337,-0$ $.0003682009 \backslash \mathrm{C}, 0,-4.7346410812,1.5390104491,0.0006005231 \backslash \mathrm{C}, 0,-3.5085137$ $164,0.8956338201,0.0030605252 \backslash \mathrm{C}, 0,-2.2888972292,1.4885017133,0.0027955$ $52 \backslash \mathrm{H}, 0,-5.671636113,0.9917414591,0.0010075156 \backslash \mathrm{S}, 0,-0.7407446154,3.6485$ $894008,-0.0006356875 \backslash \mathrm{H}, 0,-3.5256598893,4.7197959337,-0.0053652736 \backslash \mathrm{H}, 0$, $-5.6599324293,3.4991561785,-0.0044976018 \backslash \mathrm{C}, 0,-0.9864156486,5.443142341$ $8,-0.0047188528 \backslash \mathrm{H}, 0,0.0204521282,5.8642279452,-0.0048092809 \backslash \mathrm{H}, 0,-1.517$ $913466,5.7544000697,0.8966103249 \backslash \mathrm{H}, 0,-1.5164394425,5.7504931396,-0.908$ $2547174 \backslash \mathrm{H}, 0,-1.356063073,0.9336993401,0.0048107862 \backslash \backslash$ Version=EM64L-G09R evB.01 \State=3-A \MP2/GTBas $1=-667.2831313 \backslash \mathrm{CCSD}(\mathrm{T}) / \mathrm{GTBas} 1=-667.4060508 \backslash \mathrm{M}$ P2 / GTBas2=0 . \MP2/GTBas3=0. \HF/GTMP2LargeXP=-66 . 4533769 \MP2/GTMP2Large $\mathrm{XP}=-667.6560197 \backslash \mathrm{HF} / \mathrm{GFHFB} 3=-666.4603929 \backslash \mathrm{HF} / \mathrm{GFHFB} 4=-666.4838309 \backslash \mathrm{G} 4 \mathrm{MP} 2=-6$ $67.8930393 \backslash$ FreqCoord $=-6.6605161891,6.8742806376,-0.0056400856,-8.92388$ $36082,5.5829880585,-0.0047262188,-4.3324168257,5.5265901219,-0.0006957$ $989,-8.9471749809,2.9083082644,0.0011348241,-6.6301300575,1.6925026353$ $, 0.0057835545,-4.3253889095,2.8128605865,0.0052828276,-10.7178389789,1$ $.8741197522,0.0019039286,-1.3998044575,6.8948347389,-0.0012012753,-6.6$ $625316285,8.9191217178,-0.0101388977,-10.6957222219,6.6124468736,-0.00$ $84992357,-1.864055429,10.2860483283,-0.0089173395,0.0386489212,11.0817$ $847973,-0.0090882238,-2.8684407441,10.8742401908,1.6943479619,-2.86565$ $52434,10.8668571629,-1.7163526748,-2.5625878268,1.7644360433,0.0090910$ $684 \backslash \mathrm{PG}=\mathrm{C} 01 \quad[\mathrm{X}(\mathrm{C} 7 \mathrm{H} 7 \mathrm{~S} 1)] \backslash \mathrm{NImag}=0 \backslash \backslash$

3anti-o-thiomethoxyphenyl cation

$\backslash \backslash 1,3 \backslash \mathrm{C}, 0,-3.5477953793,3.6750796509,-0.0034311039 \backslash \mathrm{C}, 0,-4.7$

$187132238,2.952056643,-0.0026910188 \backslash C, 0,-2.3064951786,2.9714644719,-0$. $0011453671 \backslash \mathrm{C}, 0,-4.713855259,1.5359066276,0.0002850845 \backslash \mathrm{C}, 0,-3.506251348$ $4,0.8129535429,0.0026198434 \backslash \mathrm{C}, 0,-2.3720162039,1.5653348355,0.001795708$ $2 \backslash \mathrm{H}, 0,-5.6572123931,0.9990422126,0.0007856925 \backslash \mathrm{S}, 0,-0.737201247,3.64542$ $02419,-0.0014814793 \backslash \mathrm{H}, 0,-3.5676199483,4.7579160706,-0.0056984754 \backslash \mathrm{H}, 0,-$ $5.6688337742,3.473874406,-0.0044058901 \backslash \mathrm{C}, 0,-0.9612927308,5.4428021857$, $-0.0043573901 \backslash \mathrm{H}, 0,0.0494155571,5.8543458513,-0.0048183691 \backslash \mathrm{H}, 0,-1.49006$ $53361,5.7589927815,0.8970580236 \backslash \mathrm{H}, 0,-1.4897367914,5.7561365047,-0.9069$ $621451 \backslash \mathrm{H}, 0,-3.4940467433,-0.2709660261,0.0049068868 \backslash \backslash$ Version=EM64L-G09 RevB.01 \State=3-A \MP2/GTBas $1=-667.274637 \backslash \operatorname{CCSD}(\mathrm{T}) / \mathrm{GTB} a \mathrm{~s} 1=-667.4086831 \backslash \mathrm{M}$ $\mathrm{P} 2 / \mathrm{GTB} a \mathrm{~s} 2=0 . \backslash \mathrm{MP} 2 / \mathrm{GTBas} 3=0 . \backslash \mathrm{HF} / \mathrm{GTMP} 2 \mathrm{LargeXP}=-666.4685772 \backslash \mathrm{MP} 2 / \mathrm{GTMP} 2 \mathrm{Large}$ $\mathrm{XP}=-667.6456378 \backslash \mathrm{HF} / \mathrm{GFHFB} 3=-666.4756624 \backslash \mathrm{HF} / \mathrm{GFHFB} 4=-666.4990416 \backslash \mathrm{G} 4 \mathrm{MP} 2=-6$ $67.893605 \backslash$ FreqCoord=-6.7043616423,6.9448940568,-0.0064838466,-8.917075 
$6927,5.5785785841,-0.0050852886,-4.3586442144,5.6152540654,-0.00216443$ $02,-8.9078954696,2.9024428918,0.0005387316,-6.6258548015,1.5362595549$, $0.0049507866,-4.4824610081,2.9580541454,0.0033933968,-10.6905820984,1$. $8879161769,0.0014847436,-1.3931084617,6.8888458964,-0.0027995902,-6.74$ $18246486,8.9911583367,-0.0107685578,-10.7125433261,6.5646712475,-0.008$ $3259256,-1.8165799947,10.2854055264,-0.008234274,0.0933818696,11.06311$ $03461,-0.0091053981,-2.8158154053,10.8829191584,1.69519399,-2.81519454$ $58,10.8775215774,-1.7139100672,-6.6027914403,-0.5120515806,0.009272672$ $2 \backslash \mathrm{PG}=\mathrm{C} 01 \quad[\mathrm{X}(\mathrm{C} 7 \mathrm{H} 7 \mathrm{~S} 1)] \backslash \mathrm{NImag}=0 \backslash \backslash$

3anti-m-methoxyphenyl cation

$\backslash \backslash 1,3 \backslash \mathrm{C}, 0,-3.5650333706,3.769809059,-0.0030057741 \backslash \mathrm{C}, 0,-4.70$

$54211846,3.0082125029,-0.0023379602 \backslash \mathrm{C}, 0,-2.2778506353,3.1289110669,-0$. $000827769 \backslash \mathrm{C}, 0,-4.621183193,1.5856182806,0.0004953996 \backslash \mathrm{C}, 0,-3.3470348886$ $, 1.019581378,0.002537816 \backslash \mathrm{C}, 0,-2.1762051238,1.6876427898,0.0020858163 \backslash \mathrm{H}$ $, 0,-5.5200395581,0.9774706197,0.0010215711 \backslash 0,0,-1.1422971079,3.7610290$ $78,-0.0012401574 \backslash \mathrm{H}, 0,-3.6274615464,4.8501023806,-0.0051258539 \backslash \mathrm{H}, 0,-5.6$ $806733902,3.4824375022,-0.0039533585 \backslash \mathrm{C}, 0,-1.0414175168,5.2117145303,-0$ $.0041672422 \backslash \mathrm{H}, 0,0.0270535427,5.4140765335,-0.0043179009 \backslash \mathrm{H}, 0,-1.5093682$ $941,5.6123128015,0.8974152713 \backslash \mathrm{H}, 0,-1.5089466457,5.6087125254,-0.907552$ $3583 \backslash \mathrm{H}, 0,-1.1934910876,1.2287889515,0.0037025002 \backslash \backslash$ Version=EM64L-G0 9ReV B. $01 \backslash$ State $=3-A \backslash M P 2 / G T B a s 1=-344.6660727 \backslash \mathrm{CCSD}(\mathrm{T}) / \mathrm{GTBas} 1=-344.7766552 \backslash \mathrm{MP} 2$ /GTBas2=0 . \MP2 / GTBas3=0 . \HF / GTMP2LargeXP=-343 . 7851736 \MP2 / GTMP2LargeXP $=-345.0371886 \backslash \mathrm{HF} / \mathrm{GFHFB} 3=-343.7882906 \backslash \mathrm{HF} / \mathrm{GFHFB} 4=-343.8133594 \backslash \mathrm{G} 4 \mathrm{MP} 2=-345$ $.256446 \backslash$ FreqCoord $=-6.736936725,7.1239066947,-0.0056800898,-8.891957378$ $8,5.6846977799,-0.0044181045,-4.3045138722,5.9127850107,-0.0015642568$, $-8.7327706446,2.9963843017,0.0009361696,-6.3249792967,1.9267295746,0.0$ $047957772,-4.112431693,3.1891826828,0.0039416217,-10.4313630074,1.8471$ $517742,0.0019304895,-2.1586286964,7.1073149352,-0.0023435578,-6.854908$ $8802,9.1653652158,-0.00968646,-10.7349169578,6.5808531541,-0.007470765$ $,-1.9679938968,9.8487131451,-0.0078749465,0.0511237866,10.2311219108,-$ $0.0081596501,-2.8522927095,10.605734167,1.6958690902,-2.8514959095,10$. $5989306312,-1.7150254084,-2.2553712977,2.3220745935,0.0069967113 \backslash \mathrm{PG}=\mathrm{C} 0$ $1[\mathrm{X}(\mathrm{C} 7 \mathrm{H} 7 \mathrm{O} 1)] \backslash \mathrm{NImag}=0 \backslash \backslash$

${ }^{3}$ anti-o-methoxyphenyl cation

$\backslash \backslash 1,3 \backslash \mathrm{C}, 0,-3.5668724072,3.7928282423,-0.0030029233 \backslash \mathrm{C}, 0,-4.6$

$845002361,3.0000999717,-0.0023215582 \backslash \mathrm{C}, 0,-2.2811344079,3.160475692,-0$. $0011926388 \backslash \mathrm{C}, 0,-4.5957310058,1.5750723409,0.0001190412 \backslash \mathrm{C}, 0,-3.34417995$ $62,0.9239205689,0.0019657659 \backslash \mathrm{C}, 0,-2.2565058049,1.7342516584,0.00125155$ $98 \backslash \mathrm{H}, 0,-5.507797127,0.9866058259,0.000561329 \backslash \mathrm{H}, 0,-3.2706467593,-0.1580$ $029603,0.0038340914 \backslash 0,0,-1.1329486188,3.7651164554,-0.0015335938 \backslash \mathrm{H}, 0,-$ $3.6470413417,4.8726889885,-0.0048134647 \backslash \mathrm{H}, 0,-5.6669445487,3.4586361986$ $,-0.0036469333 \backslash \mathrm{C}, 0,-1.0236352305,5.2150420947,-0.0041150833 \backslash \mathrm{H}, 0,0.0457$ $731694,5.4116033703,-0.0045641983 \backslash \mathrm{H}, 0,-1.4909986463,5.616739448,0.8974$ $799933 \backslash \mathrm{H}, 0,-1.4911970792,5.6136421047,-0.906971387 \backslash \backslash$ Version=EM64L-G09R

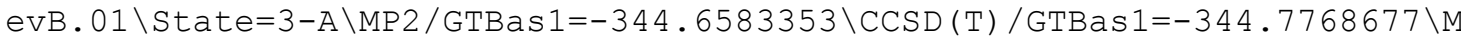
P2 / GTBas2=0 . \MP2/GTBas3=0 . \HF/GTMP2LargeXP=-343.795632 \MP2 / GTMP2LargeX $\mathrm{P}=-345.0282864 \backslash \mathrm{HF} / \mathrm{GFHFB} 3=-343.7988455 \backslash \mathrm{HF} / \mathrm{GFHFB} 4=-343.8238789 \backslash \mathrm{G} 4 \mathrm{MP} 2=-34$ $5.255272 \backslash$ FreqCoord $=-6.7404120005,7.1674066471,-0.0056747026,-8.8524225$ $157,5.6693673177,-0.0043871091,-4.3107193033,5.9724335075,-0.002253760$ $7,-8.6846729813,2.9764553639,0.0002249552,-6.3195842563,1.7459568437,0$ $.0037147592,-4.2641779885,3.2772606799,0.0023651053,-10.4082281654,1.8$ $64414812,0.0010607582,-6.1806266524,-0.2985823232,0.0072453827,-2.1409$ $626122,7.1150389591,-0.0028980723,-6.8919093311,9.208047719,-0.0090961$ $301,-10.7089732073,6.5358752086,-0.0068917051,-1.9343902456,9.85500133$ $05,-0.0077763804,0.0864987544,10.2264483097,-0.0086250849,-2.817579106$ 
$, 10.6140993165,1.695991397,-2.8179540898,10.608246186,-1.7139275318 \backslash \mathrm{PG}$ $=\mathrm{C} 01 \quad[\mathrm{X}(\mathrm{C} 7 \mathrm{H} 7 \mathrm{O} 1)] \backslash \mathrm{NImag}=0 \backslash \backslash$ 